\title{
Estudo de algoritmos de Otimização Estocástica aplicados em Aprendizado de Máquina
}

\author{
Jessica Katherine De Sousa Fernandes \\ DisSERTAÇÃO APRESENTADA \\ $\mathrm{AO}$ \\ Instituto DE MATEMÁticA E EstatísticA \\ DA \\ UNIVERSIDADE DE SÃO PAULO \\ PARA \\ OBTENÇÃO DO TÍTULO \\ $\mathrm{DE}$ \\ Mestre EM CiênCIAS
}

\author{
Programa: Mestrado em Ciências da Computação \\ Orientador: Prof. Dr. Ernesto G. Birgin
}

Durante o desenvolvimento deste trabalho a autora recebeu auxílio financeiro do CNPq 


\section{Estudo de algoritmos de Otimização Estocástica aplicados em Aprendizado de Máquina}

Esta versão da dissertação contém as correções e alterações sugeridas pela Comissão Julgadora durante a defesa da versão original do trabalho, realizada em 23/08/2017. Uma cópia da versão original está disponível no

Instituto de Matemática e Estatística da Universidade de São Paulo.

Comissão Julgadora:

- Prof. Dr. Ernesto Julian Goldberg Birgin (orientador) - IME-USP

- Prof. Dr. José Mario Martinez - UNICAMP

- Profa. Dra. Natasa Krejić - University of Novi Sad (Servia) 


\section{Agradecimentos}

Primeiramente, agradeço aos meus pais e irmãos por me apoiar nesta decisão de realizar um mestrado longe de casa. Sem vocês nada disto teria sido possível.

Agradeço também ao meu orientador, Ernesto G. Birgin pela paciencia, ajuda e comprensão em todo o trajeto de pesquisa.

Ao professor Marcos Raydan, muito obrigada por todos os conselhos e ajuda desde os inicios da minha carreira como matemática há mais de 5 anos.

Aos meus companheiros de estudo Julio Delgado, Oberlan Romão, John Gardenghi, Rafael Lobato e Antônio Deusany por toda a amizade e ajuda incondicional.

Finalmente, agradeço a Rodolfo Cerda e Marcelo Alvarez por todo o apoio na redação do trabalho. 


\section{Resumo}

FERNANDES, J. Estudo de algoritmos de Otimização Estocástica aplicados em Aprendizado de Máquina. 2017. Dissertação - Instituto de Matemática e Estatística, Universidade de São Paulo, São Paulo, 2017.

Em diferentes aplicações de Aprendizado de Máquina podemos estar interessados na minimização do valor esperado de certa função de perda. Para a resolução desse problema, Otimização estocástica e Sample Size Selection têm um papel importante. No presente trabalho se apresentam as análises teóricas de alguns algoritmos destas duas áreas, incluindo algumas variações que consideram redução da variância. Nos exemplos práticos pode-se observar a vantagem do método Stochastic Gradient Descent em relação ao tempo de processamento e memória, mas, considerando precisão da solução obtida juntamente com o custo de minimização, as metodologias de redução da variância obtêm as melhores soluções. Os algoritmos Dynamic Sample Size Gradient e Line Search with variable sample size selection apesar de obter soluções melhores que as de Stochastic Gradient Descent, a desvantagem se encontra no alto custo computacional deles.

Palavras-chave: Aprendizado de máquina, otimização estocástica, Sample size approximation, dynamic sample size selection, métodos de redução de variância. 


\section{Abstract}

FERNANDES, J. Study of algorithms of Stochastic Optimization applied in Machine Learning problems. 2017. Disertation - Instituto de Matemática e Estatística, Universidade de São Paulo, São Paulo, 2017.

In different Machine Learning's applications we can be interest in the minimization of the expected value of some loss function. For the resolution of this problem, Stochastic optimization and Sample size selection has an important role. In the present work, it is shown the theoretical analysis of some algorithms of these two areas, including some variations that considers variance reduction. In the practical examples we can observe the advantage of Stochastic Gradient Descent in relation to the processing time and memory, but considering accuracy of the solution obtained and the cost of minimization, the methodologies of variance reduction has the best solutions. In the algorithms Dynamic Sample Size Gradient and Line Search with variable sample size selection, despite of obtaining better solutions than Stochastic Gradient Descent, the disadvantage lies in their high computational cost.

Keywords: Machine Learning, stochastic optimization, sample size approximation, dynamic sample size selection, variance reduction methods. 


\section{Sumário}

$\begin{array}{ll}\text { Lista de Abreviaturas } & \text { ix }\end{array}$

Lista de Figuras $\quad$ xi

Lista de Tabelas $\quad$ xiii

1 Introdução $\quad 1$

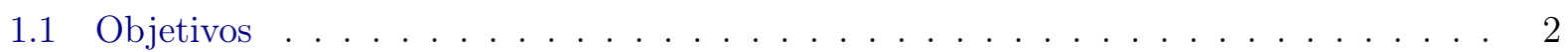

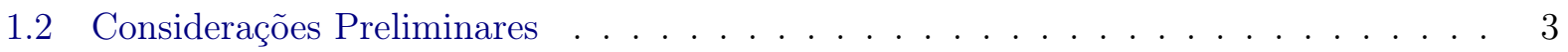

1.3 Organização do Trabalho . . . . . . . . . . . . . . . . . . . . . . . . 3

2 Conceitos $\quad 5$

2.1 Aproximação Estocástica . . . . . . . . . . . . . . . . . . . . 5

2.1.1 Descida do Gradiente Estocástico . . . . . . . . . . . . . . . . . . . . . . 9

2.2 Sample Average Approximation with Variable Sample Size . . . . . . . . . . . . . 11

2.2.1 Dynamic Sample Size Gradient . . . . . . . . . . . . . . . . . . . . . . . 12

2.2.2 Line search method with variable sample size . . . . . . . . . . . . . . . . 15

2.3 Métodos de Redução da variância . . . . . . . . . . . . . . . . . . . . . . . . . . . . . . . . .

2.3.1 Stochastic Variance Reduced Gradient . . . . . . . . . . . . . . . . . . . . . 21

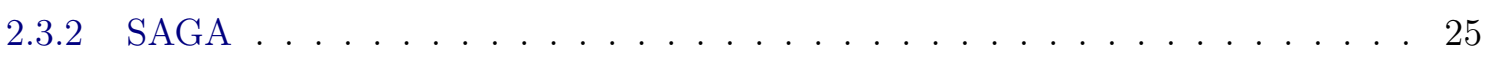

$\begin{array}{lll}3 & \text { Resultados } & \mathbf{3 7}\end{array}$

3.1 Motivação para o problema de classificação . . . . . . . . . . . . . . . 37

3.2 Mistura de Gaussianas . . . . . . . . . . . . . . . . . . . . . . . . . . . . . . 39

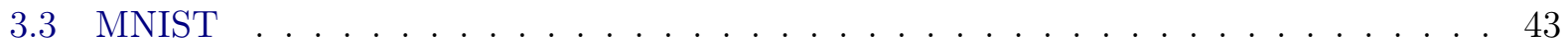

3.3.1 Seleção de hiperparâmetros . . . . . . . . . . . . . . . . . . . 43

3.3 .2 Resultados finais . . . . . . . . . . . . . . . . . . . . . . 45

4 Conclusões $\quad 49$

$\begin{array}{ll}\text { Referências Bibliográficas } & 51\end{array}$ 


\title{
Lista de Abreviaturas
}

\author{
SGD Stochastic Gradient Descent \\ DSSG Dynamic Sample Size Gradient \\ LSSS Line Search Variable Sample Size Selection
}




\section{Lista de Figuras}

3.1 Gráfico da função $\operatorname{sign}(z) \ldots \ldots \ldots$. . . . . . . . . . . . . . . . . . . . . . . . . . .

3.2 Exemplo da amostra no problema de mistura de Gaussianas . . . . . . . . . . . . . 40

3.3 Estrutura de uma rede neural de múltiplas camadas . . . . . . . . . . . . . . . . . 41

3.4 Erro de classificação nos dados de validação na seleção do parâmetro $\alpha$ no Algoritmo 2.1 .2 - SGD . . . . . . . . . . . . . . . . . . . . . 42

3.5 Desempenho dos algoritmos no problema Mistura de Gaussianas. . . . . . . . . . . . 42

3.6 Erro de classificação nos dados de validação na seleção do parâmetro $\eta$ e $m$ no Algoritmo 2.3 .1 - SVRG. . . . . . . . . . . . . . . . . . . . . . . . 45

3.7 Desempenho dos algoritmos no problema de MNIST. . . . . . . . . . . . . . . . 46 


\section{Lista de Tabelas}

3.1 Erro de classificação nas bases de treinamento, validação e teste para os dados de Mistura de Gaussianas. . . . . . . . . . . . . . . . . . . . . . . . . 42

3.2 Exemplo de dígitos corretamente classificados que fazem parte das amostras no problema MNIST . . . . . . . . . . . . . . . . . . . . . . . . . . 43

3.3 Erro de classificação nos dados de treinamento e validação na seleção do $\lambda$ no Problema $(3.7) \ldots \ldots \ldots \ldots \ldots \ldots$

3.4 Erro de classificação nos dados de validação na seleção do parâmetro $\alpha$ de tamanho do passo no Algoritmo 2.1 .2 - SGD . . . . . . . . . . . . . . . . . . . . 44

3.5 Erro de classificação nos dados de validação na seleção do parâmetro $\eta$ no Algoritmo 2.3 .2 - SAGA. . . . . . . . . . . . . . . . . . . . . 45

3.6 Erro de classificação para os dados de MNIST. . . . . . . . . . . . . . . . . . . . . 47

3.7 Exemplo de dígitos erroneamente classificados por todos os algoritmos no problema

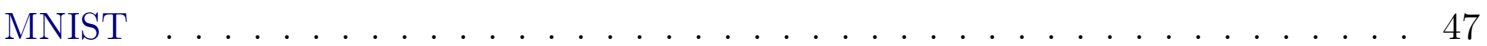




\section{Capítulo 1}

\section{Introdução}

Atualmente o análise de grandes volumes de dados para a tomada de decisões é uma área de amplo desenvolvimento. Aprendizado de Máquina [MRT12] faz referência ao conjunto de algoritmos, baseados na teoria de álgebra, probabilidades, estatística e otimização, capazes de extrair conhecimento dos dados. As aplicações do Aprendizado de máquina incluem áreas como finanças, biologia, telecomunicações ou engenharia, em problemas como detecção de fraudes, análise de riscos de crédito, reconhecimento de fala, visão computacional, classificação de imagens, entre outros. Dado que o crescimento no volume dos dados é muito acelerado, se espera que os algoritmos de resolução sejam eficientes, levando em consideração o tempo de processamento e o espaço de armazenamento.

Os métodos de Aprendizado de máquina se dividem em duas categorias: Supervisionados e Nãosupervisionados. A primeira busca o reconhecimento de padrões ou estimações de funções, onde a variável desejada (ou de saída) se encontra disponível nos dados. Alguns exemplos ressaltantes são: Regressão Linear, Logística, Máquinas de Suporte Vetorial, Redes Neurais Multicamadas, entre outros. De maneira contrária, os métodos Não-supervisionados não tem aceso à variável de saída. Neste trabalho abordaremos unicamente o caso de Aprendizado de Máquina Supervisionado. Formalmente, seja $\mathcal{X} \times \mathcal{Y}$ o espaço dos pares $(x, y)$ que seguem uma distribuição de probabilidades $P(x, y)$. De forma geral, se quer estimar os valores de $w \in \mathbb{R}^{m}$ de uma função $f_{w}(x)$ tal que se aproxime a $y$. Assim, seja $\ell\left(f_{w}(x), y\right)$ uma função de erro, se quer encontrar $w$ tal que se minimize o valor esperado de $\ell$, isto é,

$$
\left.\min _{w \in \mathbb{R}^{m}}\left\{\mathbb{E}\left(\ell\left(f_{w}(x), y\right)\right):=\int_{\mathcal{X} \times \mathcal{Y}} \ell\left(f_{w}(x), y\right)\right) d P(x, y)\right\} .
$$

Em geral, a resolução de (1.1) é difícil pois a avaliação de $\mathbb{E}\left(\ell\left(f_{w}(x), y\right)\right)$ é cara computacionalmente ou a distribuição probabilística dos dados é desconhecida.

Um possível método de resolução do problema (1.1) é por meio de Monte Carlo ou Sample Average Approximation, onde dada uma amostra $\left(x_{1}, y_{1}\right), \ldots,\left(x_{N}, y_{N}\right)$ independente e identicamente distribuída (i.e., i.i.d), a função $\mathbb{E}(\ell(w))$ (onde, $\ell(w):=\ell\left(f_{w}(x), y\right)$ ) pode ser aproximada por:

$$
\mathbb{E}(\ell(w)) \approx \hat{E}_{N}(w)=\frac{1}{N} \sum_{i=1}^{N} \ell\left(f_{w}\left(x_{i}\right), y_{i}\right) .
$$

Assim, a resolução do problema (1.1) pode ser aproximada minimizando $\hat{E}_{N}(w)$ por meio de algoritmos determinísticos. A grande desvantagem manifesta-se quando o $N$ considerado é grande e a avaliação de $\hat{E}_{N}(w)$ (e o seu gradiente ou Hessiana se é o caso, dependendo do algoritmo) é custoso e portanto, os algoritmos determinísticos resultam inadequados. Partindo disto, se consideram métodos de Programação Estocástica [Spa03] onde supomos que parte do problema a resolver pode ser representado por meio de variáveis aleatórias, pois existe incerteza em algumas das representações dos dados. Uns dos primeiros algoritmos estocásticos é o algoritmo de Robbins-Monro [RM51], onde 
se geram sequências de aproximações do minimizador da função através de:

$$
w_{k+1}=w_{k}-a_{k} \nabla \ell\left(w_{k}\right) .
$$

Este método converge se são satisfeitas certas propriedades (que serão vistas no seguinte capítulo), incluindo a seguintes características da sequência $\left\{a_{k}\right\}$ :

$$
a_{k}>0 \forall k, \sum_{k=1}^{\infty} a_{k}=\infty \text { e } \sum_{k=1}^{\infty} a_{k}^{2}<\infty .
$$

Uma escolha comum de $a_{k}$ é $\frac{a}{k^{\alpha}} \operatorname{com} a>0$ e $\frac{1}{2}<\alpha \leq 1$. Em (1.2) $\nabla \ell\left(w_{k}\right)$ representa uma estimação de $\nabla \mathbb{E}\left(\ell\left(w_{k}\right)\right)$, portanto, o algoritmo de Robbins-Monro pode ser visto como uma versão estocástica do método determinístico de descida do gradiente. Uma das desvantagens deste método é que, dependendo da escolha de $a_{k}$, a convergência pode ser lenta (o passo $a_{k}$ se torna pequeno em poucas iterações) e, em alguns dos algoritmos derivados, o viés da estimação do gradiente é grande. Assim, existem diferentes pesquisas na área para melhorar estes procedimentos. Krejić et al. [KLS13] propõem uma modificação do algoritmo de Robbins-Monro por meio de uma mistura de descida do gradiente com busca linear. Desta forma, nas primeiras iterações, onde se espera que os passos sejam maiores, usa-se a condição de Armijo, e quando se está perto da solução, consideram-se os passos do método de aproximação estocástica.

Outro tipo de enfoque para a resolução da minimização de $\hat{E}_{N}$ é a traves de Variable Sample Size Selection [BCNW12], onde em cada iteração $k$ se quer encontrar um tamanho amostral $N_{k}<N$ tal que se diminua o custo do algoritmo de otimização usado. Pode-se ver que Stochastic Approximation é um caso particular de Variable Sample Size Selection onde $N_{k}=1 \forall k$. Krejić e Krklec [KK13] propõem um algoritmo onde a cada iteração se decide aumentar ou diminuir o tamanho amostral dependendo do decrecimento de $\hat{E}_{N_{k}}$ e da amplitude do intervalo de confiança da estimação obtida. Krejić e Martínez [KM16] propõem abordar o problema de Sample Average Approximation através de Inexact Restoration, onde se transforma o problema irrestrito num problema com restrições. Assim, as avaliações aproximadas da função correspondem a inviabilidade no problema modificado.

Particularmente neste trabalho consideraremos unicamente metodologias que usam informação de primeira ordem, pois para problemas de grande porte, cálculos de ordem maior resultam mais custosos. Assim, nos enfoques mencionados, se trabalha com possíveis aproximações para o gradiente de $\mathbb{E}(\ell(w))$ ou de $\hat{E}_{N}(\ell(w))$ para o caso de Variable Sample Size Selection, mas é importante mencionar que para que uma estimação seja considerada boa, desde o ponto de vista estatístico, deve satisfazer a propriedade de consistência, isto é, seja $\hat{\theta}$ uma aproximação de $\theta$, então $\hat{\theta}$ é consistente se $\mathbb{E}(\hat{\theta})=\theta$, e a propriedade de variancia pequena, pois assim, o intervalo de confiança da estimação $\hat{\theta}$ é pequeno. Partindo destas propriedades, existem metodologias de Variance Reduction [AG07, KKK10, Rip87] onde, para a resolução do problema (1.1) se buscam estimações do gradiente de $\mathbb{E}(\ell(w))$ que diminuam a variancia das aproximações já propostas com Stochastic Approximation e Variable Sample Size Selection. Johnson e Zhang [JZ13] e Defazio e Bach [DBLJ14] propõem algoritmos inspirados nesta ideia, por meio de Control Variate e demonstram que a convergência pode ser melhorada em comparação com algumas propostas de Stochastic Approximation.

\section{$1.1 \quad$ Objetivos}

O objetivo fundamental deste trabalho é analisar o comportamento de algoritmos de otimização na resolução de problemas relacionados com Aprendizado de Máquina onde a dimensão seja considerada grande. Especificamente, se quer:

- Estudar alguns dos principais algoritmos de Stochastic Approximation, Variable Sample Size Selection e Variance Reduction.

- Comparar o desempenho de cada um dos algoritmos anteriores por meio de um banco de dados de um problema real com dimensão grande. 


\subsection{Considerações Preliminares}

A partir de agora, considera-se o problema geral de minimização de valor esperado definido por

$$
\min _{x \in \mathbb{R}^{d}} \mathbb{E}(F(x, \xi))
$$

onde $\xi \in \Omega$ é uma variável aleatória que segue uma distribuição de probabilidades $P$. Algumas afirmações no seguinte trabalho, incluem teoria de Estatística e Probabilidade. Todas elas se encontram enunciadas e provadas nas referências [DeG89, Ros97, Ros10].

\subsection{Organização do Trabalho}

O presente trabalho se encontra dividido da seguinte forma: o Capítulo 2.1 apresenta a fundamentação teórica de Stochastic Approximation junto com um dos algoritmos principais nessa área (Stochastic Gradient Descent). No Capítulo 2.2 inicia-se o análise de Sample Average Approximation e as ideias de Sample Size Selection. Adicionalmente, o Capítulo 2.3 inicia com algumas definições de alguns dos algoritmos de Variance Reduction.

Os resultados obtidos de todos os algoritmos enunciados encontram-se disponíveis no Capítulo 3. Finalmente, no Capítulo 4 discutimos algumas conclusões obtidas neste trabalho, analisamos as vantagens e desvantagens dos método estudados, assim como apresentamos algumas considerações do que acontece na atualidade na área de Aprendizado de Máquina. 


\section{Capítulo 2}

\section{Conceitos}

No seguinte capítulo está apressentada toda a fundamentação teórica dos algoritmos a analisar. Iniciando com Aproximação Estocástica e finalizando com Sample Average Approximation.

\subsection{Aproximação Estocástica}

Os inicios de Aproximação Estocástica nacem do trabalho de Robbins e Monro [RM51] para encontrar as raízes de uma função $g(x)$ baseados nas observações (com ruído) da mesma. Formalmente, queremos encontrar $x^{*} \in \mathcal{X} \subseteq \mathbb{R}^{p}$ tal que $g(x)=0$ com $g: \mathbb{R}^{p} \rightarrow \mathbb{R}^{p}$. Sejam $Y_{k}$ as observações obtidas de $g(x)$ definidas como:

$$
Y_{k}(x)=g(x)+e_{k}(x), k=0,1,2, \ldots
$$

onde $e_{k}(x)$ é o ruído de dimensão $p$.

Assim, modificando o algoritmo de descida para o caso de avaliações com ruido se tem que o algoritmo proposto por Robbin e Monro é:

Algoritmo 2.1.1. Algoritmo Robbins-Monro

Entrada: $x_{0}$ : ponto inicial;

$a_{k}$ tal que satisfaz Suposição 2.1.1.

Passo 1. $x_{k+1}=x_{k}-a_{k} Y_{k}\left(x_{k}\right)$

Para provar a convergência probabilística dos métodos estocásticos que vão ser propostos neste trabalho, existem duas definições necessárias:

Seja $\left\{x_{k}\right\}$ uma sequência de variáveis aleatórias e seja $x^{*}$ uma variável aleatória.

- Se $P\left(\lim _{k \rightarrow \infty} x_{k}=x^{*}\right)=1$ então dizemos que $x_{k}$ converge a $x^{*}$ com certeza (ou com probabilidade 1).

- Se $\lim _{k \rightarrow \infty} \mathbb{E}\left(\left\|x_{k}-x^{*}\right\|^{2}\right)=0$ dizemos que $x_{k}$ converge a $x^{*}$ no sentido de média quadrada.

Seja $\left\{x_{k}\right\}$ uma sequência gerada pelo Algoritmo 2.1.1 e denotemos por $\mathcal{F}_{k}$ a $\sigma$-álgebra gerada por $x_{0}, \ldots, x_{k}$. Sejam as seguintes suposições:

Suposição 2.1.1 (Passo da sequência).

$$
a_{k}>0, \sum_{k=1}^{\infty} a_{k}=\infty e \sum_{k=1}^{\infty} a_{k}^{2}<\infty .
$$


Suposição 2.1.2 (Direção de busca). Para alguma matriz B simétrica e positiva definida e todo $0<\eta<1$,

$$
\inf _{\eta<\left\|x-x^{*}\right\|<1 / \eta}\left(x-x^{*}\right)^{T} B g(x)>0 .
$$

Suposição 2.1.3 (Ruído com média zero). $\mathbb{E}\left(e_{k}(x) \mid \mathcal{F}_{k}\right)=0, \forall x, k$.

Suposição 2.1.4 (Limites da variância). $\|g(x)\|^{2}+\mathbb{E}\left(\left\|e_{k}(x)\right\|^{2} \mid \mathcal{F}_{k}\right) \leq c\left(1+\left\|x-x^{*}\right\|^{2}\right), \forall x, k$ e algum $c>0$.

A Suposição 2.1.1 garante o decrescimento eficiente dos passos em cada iteração $\left(\sum_{k=1}^{\infty} a_{k}=\infty\right)$ e que, perto da solução, a influencia do ruído seja pequena $\left(\sum_{k=1}^{\infty} a_{k}^{2}<\infty\right)$ [KK13]. A Suposição 2.1.3 permite garantir que os estimadores $Y_{k}(x)$ sejam consistentes, e finalmente, a Suposição 2.1.4 junto com a Suposição 2.1.3 limitam superiormente a variância de $\left\|Y_{k}(x)\right\|^{2}$ a $c\left(1+\left\|x-x^{*}\right\|^{2}\right)$, permitindo assim que $Y_{k}$ seja um bom estimador de $g$ quando $x$ está numa vizinhança de $x^{*}$.

Logo, por meio das suposições especificadas, é possível demonstrar a convergência do Algoritmo 2.1.1. O Lema 2.1.1 estabelece a convergência com probabilidade 1 de sequências de variáveis aleatórias não-negativas (a prova pode ser encontrada com detalhe em [RS71]). Este lema é necessário para a demonstração do Teorema 2.1.1, onde se estipula que a sequência gerada pelo Algoritmo 2.1.1 converge com probabilidade 1.

Lema 2.1.1 (Robbins-Siegmund). [RS71] Sejam v $v_{k}, u_{k}, \alpha_{k}$ e $\beta_{k}$ variáveis aleatórias não-negativas e sejam as seguintes suposições satisfeitas com probabilidade 1:

$$
\mathbb{E}\left(v_{k+1} \mid \tilde{\mathcal{F}}_{k}\right) \leq\left(1+\alpha_{k}\right) v_{k}-u_{k}+\beta_{k} \quad \forall k, \sum_{k=0}^{\infty} \alpha_{k}<\infty, \sum_{k=0}^{\infty} \beta_{k}<\infty
$$

onde $\tilde{\mathcal{F}}_{k}$ é a coleção $v_{0}, \ldots, v_{k}, u_{0}, \ldots, u_{k}, \alpha_{0}, \ldots, \alpha_{k}, \beta_{0}, \ldots, \beta_{k}$. Então, com probabilidade 1 temos que

$$
\lim _{k \rightarrow \infty} v_{k}=v, \quad \sum_{k=0}^{\infty} u_{k}<\infty,
$$

onde $v \geq 0$ é uma variável aleatória.

Teorema 2.1.1. [CHe03] Suponha que se satisfazem as Suposições 2.1.1-2.1.4 e que $x^{*}$ é a única solução de $g(x)=0$, então, $x_{k} \rightarrow x^{*}$ com probabilidade 1 quando $k \rightarrow \infty$.

Demonstração.

$$
\begin{aligned}
\left\|x_{k+1}-x^{*}\right\|^{2} & =\left\|x_{k}-a_{k} Y_{k}\left(x_{k}\right)-x^{*}\right\|^{2} \\
& =\left\|x_{k}-a_{k}\left(g\left(x_{k}\right)-e_{k}\left(x_{k}\right)\right)-x^{*}\right\|^{2} \\
& =\left\|x_{k}-x^{*}\right\|^{2}-2 a_{k}\left(x_{k}-x^{*}\right)^{T}\left(g\left(x_{k}\right)-e_{k}\left(x_{k}\right)\right)+a_{k}^{2}\left\|g\left(x_{k}\right)-e_{k}\left(x_{k}\right)\right\|^{2} .
\end{aligned}
$$


Logo, tomando o valor esperado condicional obtemos:

$$
\begin{aligned}
\mathbb{E}\left(\left\|x_{k+1}-x^{*}\right\|^{2} \mid \mathcal{F}_{k}\right)= & \mathbb{E}\left(\left\|x_{k}-x^{*}\right\|^{2}-2 a_{k}\left(x_{k}-x^{*}\right)^{T}\left(g\left(x_{k}\right)-e_{k}\left(x_{k}\right)\right)+a_{k}^{2}\left\|g\left(x_{k}\right)-e_{k}\left(x_{k}\right)\right\|^{2} \mid \mathcal{F}_{k}\right) \\
= & \mathbb{E}\left(\left\|x_{k}-x^{*}\right\|^{2} \mid \mathcal{F}_{k}\right)-2 a_{k} \mathbb{E}\left(\left(x_{k}-x^{*}\right)^{T}\left(g\left(x_{k}\right)-e_{k}\left(x_{k}\right)\right) \mid \mathcal{F}_{k}\right)+ \\
& \quad+a_{k}^{2} \mathbb{E}\left(\left\|g\left(x_{k}\right)-e_{k}\left(x_{k}\right)\right\|^{2} \mid \mathcal{F}_{k}\right) \\
= & \left\|x_{k}-x^{*}\right\|^{2}-2 a_{k}\left(x_{k}-x^{*}\right)^{T}\left(\mathbb{E}\left(g\left(x_{k}\right) \mid \mathcal{F}_{k}\right)-\mathbb{E}\left(e_{k}\left(x_{k}\right) \mid \mathcal{F}_{k}\right)\right)+ \\
& \quad+a_{k}^{2}\left(\mathbb{E}\left(\left\|g\left(x_{k}\right)\right\|^{2} \mid \mathcal{F}_{k}\right)-2 \mathbb{E}\left(g\left(x_{k}\right)^{T} e_{k}\left(x_{k}\right) \mid \mathcal{F}_{k}\right)+\mathbb{E}\left(\left\|e_{k}\left(x_{k}\right)\right\|^{2} \mid \mathcal{F}_{k}\right)\right) \\
= & \left\|x_{k}-x^{*}\right\|^{2}-2 a_{k}\left(x_{k}-x^{*}\right)^{T} g\left(x_{k}\right)+ \\
& \quad+a_{k}^{2}\left(\left\|g\left(x_{k}\right)\right\|^{2}-2 g\left(x_{k}\right)^{T} \mathbb{E}\left(e_{k}\left(x_{k}\right) \mid \mathcal{F}_{k}\right)+\mathbb{E}\left(\left\|e_{k}\left(x_{k}\right)\right\|^{2} \mid \mathcal{F}_{k}\right)\right) \\
= & \left\|x_{k}-x^{*}\right\|^{2}-2 a_{k}\left(x_{k}-x^{*}\right)^{T} g\left(x_{k}\right)+a_{k}^{2}\left(\left\|g\left(x_{k}\right)\right\|^{2}+\mathbb{E}\left(\left\|e_{k}\left(x_{k}\right)\right\|^{2} \mid \mathcal{F}_{k}\right)\right) \\
\leq & \left\|x_{k}-x^{*}\right\|^{2}-2 a_{k}\left(x_{k}-x^{*}\right)^{T} g\left(x_{k}\right)+c a_{k}^{2}\left(1+\left\|x_{k}-x^{*}\right\|^{2}\right) \\
= & \left(1+c a_{k}^{2}\right)\left\|x_{k}-x^{*}\right\|^{2}-2 a_{k}\left(x_{k}-x^{*}\right)^{T} g\left(x_{k}\right)+c a_{k}^{2} .
\end{aligned}
$$

Agora, pela suposição 2.1.2, podemos aplicar o Lema 2.1.1 obtendo que a sequência $\left\{\left\|x_{k}-x^{*}\right\|^{2}\right\}$ converge com probabilidade 1 e que $\sum_{k=0}^{\infty} a_{k}\left(x_{k}-x^{*}\right)^{T} g\left(x_{k}\right)<\infty$, $\operatorname{mas}$ como $\sum_{k=0}^{\infty} a_{k}=\infty$, então, $\liminf _{k \rightarrow \infty}\left(x_{k}-x^{*}\right)^{T} g\left(x_{k}\right)=0$ que, junto com a suposição 2.1.2 implicam que $x_{k} \rightarrow x^{*}$.

O seguinte teorema, enunciado e provado por Polyak [Pol87], estipula que sobre certas condições nenhum método na forma do Algoritmo 2.1.1 converge assintoticamente (no sentido de média quadrada) mais rápido que $O(1 / k)$.

Teorema 2.1.2. [PoL87] Seja $e_{k}$ ruído independente e aleatório tal que se satisfaz a Suposição 2.1.3 $e \mathbb{E}\left[\left\|e_{k}\right\|^{2}\right] \geq \delta^{2}, \forall k$. Suponha que existe um único ponto $x^{*}$ tal que $g\left(x^{*}\right)=0$ e que $g(x)$ satisfaz $\|g(x)\| \leq L\left\|x-x^{*}\right\| \forall x$. Então,

$$
\mathbb{E}\left(\left\|x_{k}-x^{*}\right\|^{2}\right) \geq \frac{1}{a+k b}
$$

onde $a=1 / \mathbb{E}\left(\left\|x_{0}-x^{*}\right\|^{2}\right)$ e $b={ }^{2} / \delta^{2}$.

Demonstração.

$$
\begin{aligned}
\mathbb{E}\left(\left\|x_{k+1}-x^{*}\right\|^{2} \mid \mathcal{F}_{k}\right)= & \mathbb{E}\left(\left\|x_{k}-a_{k} Y_{k}\left(x_{k}\right)-x^{*}\right\|^{2} \mid \mathcal{F}_{k}\right) \\
= & \mathbb{E}\left(\left\|x_{k}-x^{*}-a_{k}\left(g\left(x_{k}\right)+e_{k}\left(x_{k}\right)\right)\right\|^{2} \mid \mathcal{F}_{k}\right) \\
= & \mathbb{E}\left(\left\|x_{k}-x^{*}-a_{k} g\left(x_{k}\right)\right\|^{2} \mid \mathcal{F}_{k}\right)+a_{k}^{2} \mathbb{E}\left(\left\|e_{k}\left(x_{k}\right)\right\|^{2} \mid \mathcal{F}_{k}\right)+ \\
& -2 a_{k} \mathbb{E}\left(\left(x_{k}-x^{*}-a_{k} g\left(x_{k}\right)\right)^{T} e_{k}\left(x_{k}\right) \mid \mathcal{F}_{k}\right) .
\end{aligned}
$$

Logo, pelas propriedades do valor esperado temos

$$
\mathbb{E}\left(\left(x_{k}-x^{*}-a_{k} g\left(x_{k}\right)\right)^{T} e_{k}\left(x_{k}\right) \mid \mathcal{F}_{k}\right)=\left(x_{k}-x^{*}-a_{k} g\left(x_{k}\right)\right)^{T} \mathbb{E}\left(e_{k}\left(x_{k}\right) \mid \mathcal{F}_{k}\right)=0 .
$$

Assim, substituindo em (2.1) e pelas propriedades de valor esperado, temos que:

$$
\mathbb{E}\left(\left\|x_{k+1}-x^{*}\right\|^{2} \mid \mathcal{F}_{k}\right)=\left\|x_{k}-x^{*}-a_{k} g\left(x_{k}\right)\right\|^{2}+a_{k}^{2} \mathbb{E}\left(\left\|e_{k}\left(x_{k}\right)\right\|^{2} \mid \mathcal{F}_{k}\right) .
$$


Pela propriedade triangular reversa se tem que:

$$
\begin{aligned}
\left\|x_{k}-x^{*}-a_{k} g\left(x_{k}\right)\right\| & \geq\left|\left\|x_{k}-x^{*}\right\|-a_{k}\left\|g\left(x_{k}\right)|\||\right.\right. \\
& \geq\left|\left\|x_{k}-x^{*}\right\|-a_{k} L\left\|x_{k}-x^{*}\right\|\right| \\
& =\left|1-a_{k} L\right|\left\|x_{k}-x^{*}\right\| .
\end{aligned}
$$

Então,

$$
\left\|x_{k}-x^{*}-a_{k} g\left(x_{k}\right)\right\|^{2} \geq\left(1-a_{k} L\right)^{2}\left\|x_{k}-x^{*}\right\|^{2} .
$$

Logo, pelas propriedades do valor esperado temos que $\mathbb{E}(Y)=\mathbb{E}(\mathbb{E}(Y \mid X))$. Assim, aplicando-a em (2.2) com (2.3) temos:

$$
\begin{aligned}
\mathbb{E}\left(\left\|x_{k+1}-x^{*}\right\|^{2}\right) & \geq\left(1-a_{k} L\right)^{2} \mathbb{E}\left(\left\|x_{k}-x^{*}\right\|^{2}\right)+a_{k}^{2} \mathbb{E}\left(\left\|e_{k}\left(x_{k}\right)\right\|^{2}\right) \\
& \geq\left(1-a_{k} L\right)^{2} \mathbb{E}\left(\left\|x_{k}-x^{*}\right\|^{2}\right)+a_{k}^{2} \delta^{2} .
\end{aligned}
$$

Seja agora $F\left(a_{k}\right)=\left(1-a_{k} L\right)^{2} \mathbb{E}\left(\left\|x_{k}-x^{*}\right\|^{2}\right)+a_{k}^{2} \delta^{2}$. Procurando os pontos estacionários de $F$ temos que

$$
F_{a_{k}}^{\prime}=-2 L\left(1-a_{k} L\right) \mathbb{E}\left(\left\|x_{k}-x^{*}\right\|^{2}\right)+2 a_{k} \delta^{2}=0,
$$

portanto,

$$
a^{*}=\frac{L^{2} \mathbb{E}\left(\left\|x_{k}-x^{*}\right\|^{2}\right)}{L^{2} \mathbb{E}\left(\left\|x_{k}-x^{*}\right\|^{2}\right)+\delta^{2}}
$$

é o único ponto estacionário, que é minimizador pois $F_{a_{k}}^{\prime \prime}=2 L^{2} \mathbb{E}\left(\left\|x_{k}-x^{*}\right\|^{2}\right)+2 \delta^{2}>0$.

Assim, voltando a (2.4) temos que

$$
\begin{aligned}
\mathbb{E}\left(\left\|x_{k+1}-x^{*}\right\|^{2}\right) & \geq\left(1-a_{k} L\right)^{2} \mathbb{E}\left(\left\|x_{k}-x^{*}\right\|^{2}\right)+a_{k}^{2} \delta^{2} \\
& \geq\left(1-a^{*} L\right)^{2} \mathbb{E}\left(\left\|x_{k}-x^{*}\right\|^{2}\right)+\left(a^{*}\right)^{2} \delta^{2} \\
& =\left(1-\frac{L^{2} \mathbb{E}\left(\left\|x_{k}-x^{*}\right\|^{2}\right)}{L^{2} \mathbb{E}\left(\left\|x_{k}-x^{*}\right\|^{2}\right)+\delta^{2}}\right)^{2} \mathbb{E}\left(\left\|x_{k}-x^{*}\right\|^{2}\right)+\frac{\delta^{2} L^{2} \mathbb{E}\left(\left\|x_{k}-x^{*}\right\|^{2}\right)^{2}}{\left(L^{2} \mathbb{E}\left(\left\|x_{k}-x^{*}\right\|^{2}\right)+\delta^{2}\right)^{2}} \\
& =\left[\delta^{4}+\delta^{2} L^{2} \mathbb{E}\left(\left\|x_{k}-x^{*}\right\|^{2}\right)\right] \frac{\mathbb{E}\left(\left\|x_{k}-x^{*}\right\|^{2}\right)}{\left(L^{2} \mathbb{E}\left(\left\|x_{k}-x^{*}\right\|^{2}\right)+\delta^{2}\right)^{2}} \\
& =\left[\delta^{2}+L^{2} \mathbb{E}\left(\left\|x_{k}-x^{*}\right\|^{2}\right)\right] \frac{\delta^{2} \mathbb{E}\left(\left\|x_{k}-x^{*}\right\|^{2}\right)}{\left(L^{2} \mathbb{E}\left(\left\|x_{k}-x^{*}\right\|^{2}\right)+\delta^{2}\right)^{2}} \\
& =\frac{\delta^{2} \mathbb{E}\left(\left\|x_{k}-x^{*}\right\|^{2}\right)}{L^{2} \mathbb{E}\left(\left\|x_{k}-x^{*}\right\|^{2}\right)+\delta^{2}} .
\end{aligned}
$$

Assim, reescrevendo obtemos

$$
\mathbb{E}\left(\left\|x_{k+1}-x^{*}\right\|^{2}\right) \geq\left[\frac{L^{2}}{\delta^{2}}+\frac{1}{\mathbb{E}\left(\left\|x_{k}-x^{*}\right\|^{2}\right)}\right]^{-1} .
$$


Portanto,

$$
\frac{1}{\mathbb{E}\left(\left\|x_{k+1}-x^{*}\right\|^{2}\right)} \leq \frac{L^{2}}{\delta^{2}}+\frac{1}{\mathbb{E}\left(\left\|x_{k}-x^{*}\right\|^{2}\right)} .
$$

Denotemos por $u_{k}=\frac{1}{\mathbb{E}\left(\left\|x_{k}-x^{*}\right\|^{2}\right)}$. Logo,

$$
u_{k+1} \leq \frac{L^{2}}{\delta^{2}}+u_{k}
$$

Assim, tomando a soma em (2.6) temos

$$
\begin{aligned}
& \sum_{j=0}^{k-1}\left(u_{k+1}-u_{k}\right) \leq \sum_{j=0}^{k-1} \frac{L^{2}}{\delta^{2}} \\
& u_{k}-u_{0} \leq k \frac{L^{2}}{\delta^{2}} \\
& \frac{1}{\mathbb{E}\left(\left\|x_{k}-x^{*}\right\|^{2}\right)} \leq k \frac{L^{2}}{\delta^{2}}+\frac{1}{\mathbb{E}\left(\left\|x_{0}-x^{*}\right\|^{2}\right)} .
\end{aligned}
$$

Finalmente,

$$
\mathbb{E}\left(\left\|x_{k}-x^{*}\right\|^{2}\right) \geq\left[k \frac{L^{2}}{\delta^{2}}+\frac{1}{\mathbb{E}\left(\left\|x_{0}-x^{*}\right\|^{2}\right)}\right]^{-1}
$$

\subsubsection{Descida do Gradiente Estocástico}

A proposta de Robbins e Monro pode ser aplicada para encontrar pontos estacionários do problema geral

$$
\min _{x \in \mathbb{R}^{d}}\{f(x)=\mathbb{E}(F(x, \xi))\}
$$

se consideramos

$$
g(x)=\nabla f(x)
$$

Por definição sabemos que

$$
\mathbb{E}(F(x, \xi))=\int_{\Omega} F(x, \nu) P_{\xi}(\nu \mid x) d \nu .
$$

Se supomos que $\xi$ é independente de $x$ então a expressão anterior pode ser reescrita como:

$$
\mathbb{E}(F(x, \xi))=\int_{\Omega} F(x, \nu) P_{\xi}(\nu) d \nu .
$$

Queremos encontrar estimadores $Y(x)$ sem viés de $g(x)$ (i.e. $\mathbb{E}(Y(x))=g(x)$ ). Logo, se $g(x)$ satisfaz que:

$$
g(x)=\frac{\partial}{\partial x} \int_{\Omega} F(x, \nu) P_{\xi}(\nu) d \nu=\int_{\Omega} \frac{\partial F(x, \nu)}{\partial x} P_{\xi}(\nu) d \nu
$$

obtemos que um possível estimador sem viés de $g(x)$ é $Y(x)=\partial F(x, \xi) / \partial x$.

Em geral a propriedade descrita em (2.8) sobre $g(x)$ não é simples de satisfazer. O seguinte teorema estipula uma condição suficiente para que a intercambiabilidade entre o gradiente e a integral seja válida. 
Teorema 2.1.3 (Lebesgue Dominated Convergence Theorem). [SPA03] Suponha que $\mathcal{X}$ é um conjunto aberto. Sejam $H$ e $\partial H / \partial x$ funções continuas em $\mathcal{X} \times \Omega$. Suponha que existem funções não negativas $q_{0}(\xi)$ e $q_{1}(\xi)$ tais que

$$
|H(x, \xi)| \leq q_{0}(\xi) \text { e }\left\|\frac{\partial H(x, \xi)}{\partial x}\right\| \leq q_{1}(\xi) \forall(x, \xi) \in \mathcal{X} \times \Omega,
$$

onde $\int_{\Omega} q_{0}(\xi) d \xi<\infty$ e $\int_{\Omega} q_{1}(\xi) d \xi<\infty$. Então,

$$
\frac{\partial}{\partial x} \int_{\Omega} H(x, \xi) d \xi=\int_{\Omega} \frac{\partial H(x, \xi)}{\partial x} d \xi .
$$

Por conta da definição do estimador $Y(x)$ de $g(x)$ e supondo que $F$ é conhecida e derivável, o Algoritmo 2.1.1 pode ser modificado como segue:

Algoritmo 2.1.2. Stochastic Gradient Descent

Entrada: $x_{0}$ : ponto inicial;

$a_{k}$ tal que satisfaz Suposição 2.1.1;

$k=0$.

Passo 1. Selecione $\xi_{k}$ aleatoriamente

Passo 2. $x_{k+1}=x_{k}-\left.a_{k} \frac{\partial F\left(x, \xi_{k}\right)}{\partial x}\right|_{x=x_{k}}$

Passo 3. $k \leftarrow k+1$

Passo 4. Volte ao Passo 1 até satisfazer algum critério de parada

As seguintes suposições são necessárias para a demonstração de convergência do Algoritmo 2.1.2:

Suposição 2.1.5 (Convexidade). A função $F$ é convexa e $\mathbb{E}(F(x, \xi))<\infty \forall x \in \mathbb{R}^{d}$.

Suposição 2.1.6. O erro $e_{k}(x)$ satisfaz $\sum_{k=0}^{\infty} a_{k}^{2} \mathbb{E}\left(\left\|e_{k}\left(x_{k}\right)\right\|^{2} \mid \mathcal{F}_{k}\right)<\infty$ com probabilidade 1 .

A Suposição 2.1.5 junto com o fato de que estamos considerando $F$ diferenciável, garante que o Teorema de intercambialidade da integral e da derivada é satisfeito (Teorema 2.1.3), i.e., $\nabla f(x)=$ $\mathbb{E}\left(\nabla_{x} F(x, \xi)\right)$. A Suposição 2.1.6 pode ser substituída pela suposição 2.1.4, pois esta última garante que $\mathbb{E}\left(\left\|e_{k}\left(x_{k}\right)\right\|^{2}\right)$ está limitada e portanto a Suposição 2.1.6 é satisfeita. O seguinte teorema garante a convergência com probabilidade 1 do Algoritmo 2.1.2.

Teorema 2.1.4. [YNS12] Suponha que as Suposições 2.1.1,2.1.3, 2.1.5 e 2.1.6 são satisfeitas e seja $f$ diferenciável com gradiente Lipschitz com constante L. Suponha que o conjunto $\mathcal{X}^{*}$ de soluções ótimas do problema (2.7) não é vazio. Então a sequência $\left\{x_{k}\right\}$ gerada pelo Algoritmo 2.1.2 converge com probabilidade 1 quando $k \rightarrow \infty$ a algum ponto em $\mathcal{X}^{*}$.

Demonstração.

Seja $x^{*} \in \mathcal{X}^{*}$. Temos então,

$$
\begin{aligned}
\left\|x_{k+1}-x^{*}\right\|^{2}= & \left\|x_{k}-x^{*}-a_{k} \nabla F\left(x_{k}, \xi_{k}\right)\right\|^{2} \\
= & \left\|x_{k}-x^{*}-a_{k}\left(\nabla f\left(x_{k}\right)+e_{k}\left(x_{k}\right)\right)\right\|^{2} \\
= & \left\|x_{k}-x^{*}\right\|^{2}-2 a_{k}\left(\nabla f\left(x_{k}\right)+e_{k}\left(x_{k}\right)\right)^{T}\left(x_{k}-x^{*}\right)+a_{k}^{2}\left\|\nabla f\left(x_{k}\right)+e_{k}\left(x_{k}\right)\right\|^{2} \\
= & \left\|x_{k}-x^{*}\right\|^{2}-2 a_{k} \nabla f\left(x_{k}\right)^{T}\left(x_{k}-x^{*}\right)-2 a_{k} e_{k}\left(x_{k}\right)^{T}\left(x_{k}-x^{*}\right)+ \\
& \quad+a_{k}^{2}\left\|\nabla f\left(x_{k}\right)+e_{k}\left(x_{k}\right)\right\|^{2} .
\end{aligned}
$$


Como $f$ é convexa, temos que $f\left(x^{*}\right) \geq f\left(x_{k}\right)-\nabla f\left(x_{k}\right)^{T}\left(x_{k}-x^{*}\right)$. Assim,

$$
\begin{gathered}
\left\|x_{k+1}-x^{*}\right\|^{2} \leq\left\|x_{k}-x^{*}\right\|^{2}-2 a_{k}\left(f\left(x_{k}\right)-f\left(x^{*}\right)\right)-2 a_{k} e_{k}\left(x_{k}\right)^{T}\left(x_{k}-x^{*}\right)+ \\
\quad+a_{k}^{2}\left\|\nabla f\left(x_{k}\right)+e_{k}\left(x_{k}\right)\right\|^{2} \\
=\left\|x_{k}-x^{*}\right\|^{2}-2 a_{k}\left(f\left(x_{k}\right)-f\left(x^{*}\right)\right)-2 a_{k} e_{k}\left(x_{k}\right)^{T}\left(x_{k}-x^{*}\right)+ \\
\quad+a_{k}^{2}\left\|\nabla f\left(x_{k}\right)-\nabla f\left(x^{*}\right)+e_{k}\left(x_{k}\right)+\nabla f\left(x^{*}\right)\right\|^{2} .
\end{gathered}
$$

Logo, como $\|a+b\|^{2} \leq 2\|a\|^{2}+2\|b\|^{2}$, segue que

$$
\begin{aligned}
\left\|x_{k+1}-x^{*}\right\|^{2} \leq \| & x_{k}-x^{*} \|^{2}-2 a_{k}\left(f\left(x_{k}\right)-f\left(x^{*}\right)\right)-2 a_{k} e_{k}\left(x_{k}\right)^{T}\left(x_{k}-x^{*}\right)+ \\
& +2 a_{k}^{2}\left\|\nabla f\left(x_{k}\right)-\nabla f\left(x^{*}\right)\right\|^{2}+2 a_{k}^{2}\left\|\nabla f\left(x^{*}\right)+e_{k}\left(x_{k}\right)\right\|^{2} \\
\leq \| & x_{k}-x^{*} \|^{2}-2 a_{k}\left(f\left(x_{k}\right)-f\left(x^{*}\right)\right)-2 a_{k} e_{k}\left(x_{k}\right)^{T}\left(x_{k}-x^{*}\right)+ \\
& \quad+2 a_{k}^{2} L^{2}\left\|x_{k}-x^{*}\right\|^{2}+2 a_{k}^{2}\left\|\nabla f\left(x^{*}\right)+e_{k}\left(x_{k}\right)\right\|^{2} \\
= & \left(1+2 a_{k}^{2} L^{2}\right)\left\|x_{k}-x^{*}\right\|^{2}-2 a_{k}\left(f\left(x_{k}\right)-f\left(x^{*}\right)\right)-2 a_{k} e_{k}\left(x_{k}\right)^{T}\left(x_{k}-x^{*}\right)+ \\
& +2 a_{k}^{2}\left\|\nabla f\left(x^{*}\right)+e_{k}\left(x_{k}\right)\right\|^{2} .
\end{aligned}
$$

Agora, tomando o valor esperado condicional de (2.9) e considerando que $\nabla f\left(x^{*}\right)=0$ temos

$$
\begin{aligned}
\mathbb{E}\left(\left\|x_{k+1}-x^{*}\right\|^{2} \mid \mathcal{F}_{k}\right) \leq & \left(1+2 a_{k}^{2} L^{2}\right) \mathbb{E}\left(\left\|x_{k}-x^{*}\right\|^{2} \mid \mathcal{F}_{k}\right)-2 a_{k} \mathbb{E}\left(f\left(x_{k}\right)-f\left(x^{*}\right) \mid \mathcal{F}_{k}\right)+ \\
& \quad-2 a_{k} \mathbb{E}\left(e_{k}\left(x_{k}\right)^{T}\left(x_{k}-x^{*}\right) \mid \mathcal{F}_{k}\right)+2 a_{k}^{2} \mathbb{E}\left(\left\|e_{k}\left(x_{k}\right)\right\|^{2} \mid \mathcal{F}_{k}\right) \\
= & \left(1+2 a_{k}^{2} L^{2}\right)\left\|x_{k}-x^{*}\right\|^{2}-2 a_{k}\left(f\left(x_{k}\right)-f\left(x^{*}\right)\right)+ \\
& \quad-2 a_{k} \mathbb{E}\left(e_{k}\left(x_{k}\right) \mid \mathcal{F}_{k}\right)^{T}\left(x_{k}-x^{*}\right)+2 a_{k}^{2} \mathbb{E}\left(\left\|e_{k}\left(x_{k}\right)\right\|^{2} \mid \mathcal{F}_{k}\right) \\
= & \left(1+2 a_{k}^{2} L^{2}\right)\left\|x_{k}-x^{*}\right\|^{2}-2 a_{k}\left(f\left(x_{k}\right)-f\left(x^{*}\right)\right)+ \\
& +2 a_{k}^{2} \mathbb{E}\left(\left\|e_{k}\left(x_{k}\right)\right\|^{2} \mid \mathcal{F}_{k}\right) .
\end{aligned}
$$

Logo, pelas Suposições 2.1.1 e 2.1.6 podemos aplicar o Lema 2.1.1, obtendo que a sequência $\left\{\left\|x_{k+1}-x^{*}\right\|^{2}\right\}$ converge com probabilidade 1 e que $\sum_{k=0}^{\infty} a_{k}\left(f\left(x_{k}\right)-f\left(x^{*}\right)\right)<\infty$. Assim, como $\sum_{k=0}^{\infty} a_{k}=\infty$ então, $\liminf _{k \rightarrow \infty} f\left(x_{k}\right)-f\left(x^{*}\right)=0$, portanto existe uma subsequência de $\left\{x_{k}\right\}$ tal que $f\left(x_{k}\right) \rightarrow f\left(x^{*}\right)$. Logo, por continuidade de $f$, essa subsequência de $\left\{x_{k}\right\}$ converge a $x^{*} \in \mathcal{X}^{*}$.

No Teorema 2.1.2 foi demonstrado que os algoritmos da forma de Robbins-Monro não convergem assintoticamente mais rápido que $O(1 / k)$. Aplicando o Teorema 2.1.2 ao Algoritmo 2.1.2 supondo que $f$ é estritamente convexa com parâmetro $\mu$ e tomando um passo $a_{k}=1 / k \mu$ obtemos a convergência (no sentido de média quadrada) igual a $1 / k$. Existem varias modificações deste algoritmo, pois na maioria dos problemas, as condições de independência entre $\xi$ e $x$ ou a intercambiabilidade da integral e derivada em (2.8) não são satisfeitas, portanto a estimação feita do gradiente pode possuir viés.

\subsection{Sample Average Approximation with Variable Sample Size}

Consideremos o problema de minimização dado em (2.7). Seja $\xi_{1}, \ldots, \xi_{N}$ uma amostra de tamanho $N$ independente e identicamente distribuída (i.i.d.) de $\xi$. Por meio do método de Sample Average Approximation [KK13] (melhor conhecido como método de Monte Carlo [AG07, KKK10]) pode-se 
estimar $\mathbb{E}(F(x, \xi)$ da seguinte forma:

$$
\mathbb{E}(F(x, \xi)) \approx \frac{1}{N} \sum_{i=1}^{N} F\left(x, \xi_{i}\right) .
$$

A Lei forte de grandes números [Ros97] garante que a aproximação (2.11) converge com probabilidade 1 a $\mathbb{E}(F(x, \xi))$ quando $N \rightarrow \infty$. Assim, a resolução de (2.7) pode ser aproximada por meio de

$$
\min _{x \in \mathbb{R}^{d}}\left\{\hat{f}_{N}(x)=\frac{1}{N} \sum_{i=1}^{N} F\left(x, \xi_{i}\right)\right\} .
$$

A solução do problema (2.12) pode ser encontrada através de algoritmos determinísticos. Nos casos que estamos considerando, o $N$ é grande, e portanto a avaliação de (2.11) é custosa. Assim, os métodos que serão estudados tentam diminuir o custo do algoritmo tomando uma subamostra aleatória de $\left\{\xi_{1}, \ldots, \xi_{N}\right\}$ de tamanho $N_{k}<N$ em cada iteração $k$. Esta ideia é conhecida como Variable Sample Size Strategy [BCNW12, KK13].

Os seguintes algoritmos a apresentar, possuem a vantagem de que em cada iteração se decide aumentar (manter o diminuir, dependendo do método) o tamanho da amostra utilizada para a aproximação do valor esperado. Assim, nas primeiras iterações, a quantidade de avaliações da função $F$ (e do seu gradiente se é o caso) sera menor, logrando avances na sequência $\left\{x_{k}\right\}$, e a medida que se aumenta $k$, melhorar a estimação de $\mathbb{E}(F(x, \xi))$ com amostras maiores. É importante destacar que em alguns problemas é impossível computacionalmente avaliar (2.11) devido ao $N$ grande. É por isto que esperasse que a precisão final obtida pelo algoritmo de resolução não chegue a considerar os $N$ dados.

\subsubsection{Dynamic Sample Size Gradient}

Byrd et. al. [BCNW12] propõem um algoritmo para a resolução do problema (2.7) com a aproximação (2.11) incrementando, em cada iteração o tamanho amostral $N_{k}$ tomando em consideração uma estimação da variância do gradiente aproximado.

Seja $S$ uma amostra aleatória de $\left\{\xi_{1}, \ldots, \xi_{N}\right\}$. Definamos $\hat{f}_{S}(x)$ como a aproximação de $\mathbb{E}(F(x, \xi))$ dada por $\hat{f}_{S}(x)=\frac{1}{|S|} \sum_{i \in S} F\left(x, \xi_{i}\right)$. A ideia principal no Algoritmo 2.2.1 é a cada iteração gerar um passo do algoritmo de Descida do Gradiente para minimizar $\hat{f}_{S}(x)$, garantindo que o avanço obtido implica progresso em $\hat{f}_{N}$ também.

Seja

$$
\delta_{S}(x)=\left\|\nabla \hat{f}_{S}(x)-\nabla \hat{f}_{N}(x)\right\|_{2} .
$$

A seguinte proposição estipula uma condição sobre $\delta_{S}(x)$ que garante que a direção de descida do gradiente sobre a amostra $S$, é também de descida para $\hat{f}_{N}$, função objetivo do problema (2.12).

Proposição 2.2.1. Existe $\beta \in(0,1)$ tal que se $\delta_{S}(x) \leq \beta\left\|\nabla \hat{f}_{S}(x)\right\|_{2}$, então a direção $d=-\nabla \hat{f}_{S}(x)$ é de descida para $\hat{f}_{N}(x)$.

Demonstração.

Precisamos provar que $-\nabla \hat{f}_{N}(x)^{T} \nabla \hat{f}_{S}(x)<0$. Temos que,

$$
\left\|\nabla \hat{f}_{S}(x)-\nabla \hat{f}_{N}(x)\right\|_{2} \leq \beta\left\|\nabla \hat{f}_{S}(x)\right\|_{2},
$$

porém,

$$
\left\|\nabla \hat{f}_{S}(x)-\nabla \hat{f}_{N}(x)\right\|_{2}^{2} \leq \beta^{2}\left\|\nabla \hat{f}_{S}(x)\right\|_{2}^{2}
$$

Logo, reescrevendo o lado esquerdo da desigualdade temos,

$$
\left\|\nabla \hat{f}_{S}(x)-\nabla \hat{f}_{N}(x)\right\|_{2}^{2}=\left\|\nabla \hat{f}_{S}(x)\right\|_{2}^{2}-2 \nabla \hat{f}_{S}(x)^{T} \nabla \hat{f}_{N}(x)+\left\|\nabla \hat{f}_{N}(x)\right\|_{2}^{2} .
$$


Substituindo em (2.14) obtemos que

$$
\begin{aligned}
&\left\|\nabla \hat{f}_{S}(x)\right\|_{2}^{2}-2 \nabla \hat{f}_{S}(x)^{T} \nabla \hat{f}_{N}(x)+\left\|\nabla \hat{f}_{N}(x)\right\|_{2}^{2} \leq \beta^{2}\left\|\nabla \hat{f}_{S}(x)\right\|_{2}^{2} . \\
&-\nabla \hat{f}_{S}(x)^{T} \nabla \hat{f}_{N}(x) \leq \frac{1}{2}\left[\left(\beta^{2}-1\right)\left\|\nabla \hat{f}_{S}(x)\right\|_{2}^{2}-\left\|\nabla \hat{f}_{N}(x)\right\|_{2}^{2}\right] \\
&=-\frac{1}{2}\left[\left(1-\beta^{2}\right)\left\|\nabla \hat{f}_{S}(x)\right\|_{2}^{2}+\left\|\nabla \hat{f}_{N}(x)\right\|_{2}^{2}\right]<0
\end{aligned}
$$

Pois, sabemos que $1-\beta^{2}>0$ e como $-\nabla \hat{f}_{S}(x)$ é direção de descida para $\hat{f}_{S}(x)$, então $\left\|\nabla \hat{f}_{S}(x)\right\|_{2}^{2}>0$. Finalmente, $\left(1-\beta^{2}\right)\left\|\nabla \hat{f}_{S}(x)\right\|_{2}^{2}+\left\|\nabla \hat{f}_{N}(x)\right\|_{2}^{2}>0$, e assim se demonstra o que se desejava.

Como em [BCNW12] considera-se que $N$ é grande e portanto a avaliação de $\nabla \hat{f}_{N}(x)$ é custosa, $\delta_{S}(x)$ não pode ser calculado de forma exata. Assim, se propõe estimar $\delta_{S}(x)$ por meio da variância de $\nabla \hat{f}_{S}(x)$, pois, se supomos que $\mathbb{E}\left(\nabla \hat{f}_{S}(x)\right)=\nabla \hat{f}_{N}(x)$ para toda possível amostra $S$, temos que,

$$
\begin{aligned}
\mathbb{E}\left(\delta_{S}(x)^{2}\right) & =\mathbb{E}\left(\left\|\nabla \hat{f}_{S}(x)-\nabla \hat{f}_{N}(x)\right\|_{2}^{2}\right) \\
& =\mathbb{E}\left(\sum_{i=1}^{d}\left(\nabla_{x_{i}} \hat{f}_{S}(x)-\nabla_{x_{i}} \hat{f}_{N}(x)\right)^{2}\right) \\
& =\sum_{i=1}^{d} \mathbb{E}\left(\nabla_{x_{i}} \hat{f}_{S}(x)-\nabla_{x_{i}} \hat{f}_{N}(x)\right)^{2} \\
& =\sum_{i=1}^{d} \operatorname{Var}\left(\nabla_{x_{i}} \hat{f}_{S}(x)\right)=\left\|\operatorname{Var}\left(\nabla \hat{f}_{S}(x)\right)\right\|_{1} .
\end{aligned}
$$

Logo, seguindo o análise detalhado em [BCNW12] obtemos que

$$
\mathbb{E}\left(\delta_{S}(x)^{2}\right)=\left\|\operatorname{Var}\left(\nabla \hat{f}_{S}(x)\right)\right\|_{1} \approx \frac{\left\|\operatorname{Var}_{S}(\nabla F(x, \xi))\right\|_{1}}{|S|} .
$$

Assim, substituindo $\delta_{S}(x)$ na condição da Proposição 2.2.1 (tomada ao quadrado) pela aproximação (2.15), obtemos a seguinte condição

$$
\frac{\left\|\operatorname{Var}_{S}(\nabla F(x, \xi))\right\|_{1}}{|S|} \leq \beta^{2}\left\|\nabla \hat{f}_{S}(x)\right\|_{2}^{2} .
$$

Na prática, se uma amostra $S$ não satisfaz a equação (2.16), aumentamos o tamanho amostral de $|S|$ para $|\hat{S}|$ pela fórmula

$$
|\hat{S}|=\frac{\left\|\operatorname{Var}_{S}(\nabla F(x, \xi))\right\|_{1}}{\beta^{2}\left\|\nabla \hat{f}_{S}(x)\right\|_{2}^{2}},
$$

pois assume-se que para todo $x$

$$
\left\|\operatorname{Var}_{\hat{S}}(\nabla F(x, \xi))\right\|_{1} \simeq\left\|\operatorname{Var}_{S}(\nabla F(x, \xi))\right\|_{1} \text { e }\left\|\nabla \hat{f}_{\hat{S}}(x)\right\|_{2} \simeq\left\|\nabla \hat{f}_{S}(x)\right\|_{2} .
$$

O algoritmo proposto por Byrd et. al. [BCNW12] se encontra a seguir: 


\section{Algoritmo 2.2.1. Dynamic Sample Size Gradient}

Entrada: Sejam $x_{0} \in \mathbb{R}^{d}, \beta \in(0,1)$ e $S_{0}$ dados.

Faça $k \leftarrow 0$.

Passo 1. Calcule $d_{k}=-\nabla \hat{f}_{S_{k}}\left(x_{k}\right)$.

Passo 2. Busca Linear: encontre $\alpha_{k}>0$ tal que

$$
\hat{f}_{S_{k}}\left(x_{k}+\alpha_{k} d_{k}\right)<\hat{f}_{S_{k}}\left(x_{k}\right) .
$$

Passo 3. $x_{k+1}=x_{k}+\alpha_{k} d_{k}$.

Passo 4. $k \leftarrow k+1$.

Passo 5. Escolha uma amostra aleatória $S_{k}$ tal que $\left|S_{k}\right|=\left|S_{k-1}\right|$.

Passo 6. Calcule a variância amostral

$$
\operatorname{Var}_{S_{k}}\left(\nabla F\left(x_{k}, \xi\right)\right)=\frac{1}{\left|S_{k}\right|-1} \sum_{i \in S_{k}}\left(\nabla F\left(x_{k}, \xi_{i}\right)-\nabla \hat{f}_{S_{k}}\left(x_{k}\right)\right)^{2} .
$$

Passo 7. Se

$$
\frac{\left\|\operatorname{Var}_{S_{k}}\left(\nabla F\left(x_{k}, \xi\right)\right)\right\|_{1}}{\left|S_{k}\right|}>\beta^{2}\left\|\nabla \hat{f}_{S_{k}}\left(x_{k}\right)\right\|_{2}^{2},
$$

incremente $S_{k}$ através de

$$
\left|S_{k}\right|=\frac{\left\|\operatorname{Var}_{S_{k}}\left(\nabla F\left(x_{k}, \xi\right)\right)\right\|_{1}}{\beta^{2}\left\|\nabla \hat{f}_{S_{k}}\left(x_{k}\right)\right\|_{2}^{2}} .
$$

Passo 8. Volte ao Passo 1 até satisfazer condição de parada.

Em [BCNW12] demonstram a convergência do Algoritmo 2.2.1 supondo que $f$ é duas vezes continuamente diferenciável e uniformemente convexa. Assim, existem constantes $0<\lambda<L$ tais que

$$
\lambda\|d\|^{2} \leq d^{T} \nabla^{2} f(x) d \leq L\|d\|^{2}, \forall x, d
$$

( $\lambda$ é o parâmetro que faz referência à convexidade uniforme e $L$ ao gradiente Lipschitz). Finalmente, o seguinte teorema estabelece a convergência do Algoritmo 2.2.1 (a demonstração detalhada pode ser encontrada na referência do teorema).

Teorema 2.2.1. [BCNW12] Suponha que para todo $k$, o tamanho amostral $n_{k}=\left|S_{k}\right|$ satisfaz $n_{k} \geq a^{k}$ para algum $a>1$, e que existe uma constante $w$ tal que $\left\|\operatorname{Var}_{N}\left(\nabla F\left(x_{k}, \xi\right)\right)\right\|_{1} \leq w \forall x_{k}$. Então, a sequência $\left\{x_{k}\right\}$ gerada pelo Algoritmo 2.2.1 satisfaz

$$
\mathbb{E}\left(\hat{f}_{N}\left(x_{k}\right)-\hat{f}_{N}\left(x^{*}\right)\right) \leq C \rho^{k}, \forall k
$$

onde

$$
\rho=\max \left\{1-\frac{\lambda}{4 L}, \frac{1}{a}\right\} \text { e } C=\max \left\{\hat{f}_{N}\left(x_{0}\right)-\hat{f}_{N}\left(x^{*}\right), \frac{2 w}{\lambda}\right\}
$$

O teorema anterior supõe que o tamanho amostral a cada iteração debe aumentar de forma exponencial, isto é, $n_{k} \geq a^{k}$ para algum $a>1$. Este análise é válido, pois, como estamos considerando que a Proposição 2.2.1 é satisfeita em valor esperado, e por isso foi estimada por meio de $\left\|\operatorname{Var}\left(\nabla \hat{f}_{S}(x)\right)\right\|_{1}$, pela natureza estocástica dos dados, pode acontecer que mudando o tamanho amostral através de (2.17) a condição (2.16) com a nova amostra não seja satisfeita. 


\subsubsection{Line search method with variable sample size}

Krejić et. al [KK13] apresentam um método para a resolução do problema (2.12) por meio de uma modificação do algoritmo de Busca Linear com condição de Armijo, variando em cada iteração a função objetivo a $\hat{f}_{N_{k}}(x)=\frac{1}{N_{k}} \sum_{i \in S_{k}} F\left(x, \xi_{i}\right)$, onde $N_{k}$ é o tamanho de uma amostra aleatória de $\xi_{1}, \ldots, \xi_{N}$. A ideia principal, ao igual que no Algoritmo de Dynamic Sample Size Gradient, é em cada iteração modificar o tamanho $N_{k}$ dependendo do avanço na sequência $\left\{x_{k}\right\}$. A diferença radica em que em [KK13] permitem o aumento ou a diminuição de $N_{k}$ dependendo do comprimento do intervalo de confiança obtido e do decrescimento na função estimada.

Definamos a quantidade de decrescimento $d m_{k}$ como a redução no modelo linear, i.e.,

$$
\begin{aligned}
d m_{k} & =m_{k}^{N_{k}}\left(x_{k}\right)-m_{k}^{N_{k}}\left(x_{k+1}\right) \\
& =-\alpha_{k} d_{k}^{T} \nabla \hat{f}_{N_{k}}\left(x_{k}\right),
\end{aligned}
$$

onde $m_{k}^{N_{k}}\left(x_{k}+s\right)=\hat{f}_{N_{k}}\left(x_{k}\right)+s^{T} \nabla \hat{f}_{N_{k}}\left(x_{k}\right)$.

Seja o intervalo de confiança da estimação $\hat{f}_{N_{k}}$ definido por

$$
P\left(f\left(x_{k}\right) \in\left[\hat{f}_{N_{k}}\left(x_{k}\right)-c, \hat{f}_{N_{k}}\left(x_{k}\right)+c\right]\right)=\delta .
$$

Definamos a falta de precisão $\varepsilon_{\delta}^{N_{k}}\left(x_{k}\right)$ como uma aproximação do comprimento do intervalo de confiança (i.e, $\varepsilon_{\delta}^{N_{k}}\left(x_{k}\right) \approx c$ ) dada por:

$$
\varepsilon_{\delta}^{N_{k}}\left(x_{k}\right)=\hat{\sigma}_{N_{k}}\left(x_{k}\right) \frac{\alpha_{\delta}}{\sqrt{N_{k}}}
$$

onde

$$
\hat{\sigma}_{N_{k}}^{2}\left(x_{k}\right)=\frac{1}{N_{k}-1} \sum_{i=1}^{N_{k}}\left(F\left(x_{k}, \xi_{i}\right)-\hat{f}_{N_{k}}\left(x_{k}\right)\right)^{2}
$$

e $\alpha_{\delta}$ é o quantil da Distribuição $\operatorname{Normal}(0,1)$.

Se quer comparar a falta de precisão e quantidade de decrescimento de tal forma que diminuam na mesma proporção. Assim, se a quantidade de decrescimento é grande comparado com Falta de precisão $\left(d m_{k}>\varepsilon_{\delta}^{N_{k}}\left(x_{k}\right)\right)$, então se diminui o tamanho amostral, pois se assume que não se precisava uma amostra $N_{k}$ tão grande para melhorar a precisão. No caso contrário em que a quantidade de decrescimento é pequeno comparado com a falta de precisão $\left(d m_{k}<\varepsilon_{\delta}^{N_{k}}\left(x_{k}\right)\right)$, se aumenta o tamanho amostral pois se quer melhorar a estimação de $\hat{f}_{N_{k}}$ e ficar mais próximos da precisão desejada.

A seguir se apresentam 3 algoritmos que resumem a ideia de Krejić et. al [KK13]. O Algoritmo 2.2.2 é o algoritmo central do método proposto, nele se especifica a regra de atualização de $x_{k+1}$. O Algoritmo 2.2.3 realiza um estudo, dependendo do análise explicado, de um possível candidato a tamanho amostral da seguinte iteração, e finalmente, o Algoritmo 2.2.4 escolhe o tamanho $N_{k+1}$ e atualiza a cota inferior $N_{k}^{m i n}$ de decrescimento possível para $N_{k}$.

Analisando a estrutura do Algoritmo 2.2.2 temos que no Passo 3 quando já estamos numa solução local para o problema de minimização de $\hat{f}_{N_{k}}$ (i.e, $\left\|\nabla \hat{f}_{N_{k}}\left(x_{k}\right)\right\|=0$ ) e a amplitude do intervalo de confiança da estimação é igual a zero $\left(\varepsilon_{\delta}^{N_{k}}=0\right)$ o tamanho amostral aumenta numa unidade, pois a certeza pela qual afirmamos que o $f(x)$ é exatamente $\hat{f}_{N_{k}}(x)$ é baixa. No caso em que $\varepsilon_{\delta}^{N_{k}}>0$ e $\left\|\nabla \hat{f}_{N_{k}}\left(x_{k}\right)\right\|=0$, o tamanho aumenta ao $N_{\max }$ pois com probabilidade $\delta$ o $f\left(x_{k}\right)$ pertence a esse intervalo obtido. Basicamente, de forma geral, quando com probabilidade alta o $f(x)$ pertence ao intervalo $\left[\hat{f}_{N_{k}}\left(x_{k}\right)-\varepsilon_{\delta}^{N_{k}}, \hat{f}_{N_{k}}\left(x_{k}\right)+\varepsilon_{\delta}^{N_{k}}\right]$, decidimos aumentar a precisão da nossa aproximação a $N_{\text {max }}$ dados. 
Algoritmo 2.2.2. Line SEARCH METHOD WITH VARIABLE SAMPLE SIZE

Entrada: Sejam $x_{0} \in \mathbb{R}^{d}, N_{0}^{\min }, N_{\max } \in \mathbb{R}, \delta, \eta, \beta, \gamma_{3}, \nu_{1} \in(0,1)$ e $\eta_{0}<1$ dados.

$k \leftarrow 0$.

$N_{k} \leftarrow N_{0}^{\min }$.

Passo 1. Gere a amostra $\xi_{1}, \ldots, \xi_{N_{\max }}$.

Passo 2. Avalie $\hat{f}_{N_{k}}\left(x_{k}\right)$ e calcule $\varepsilon_{\delta}^{N_{k}}$ usando (2.19).

Passo 3. Se $\left\|\nabla \hat{f}_{N_{k}}\left(x_{k}\right)\right\|=0$ e $N_{k}<N_{\max }$

3.1. Se $\varepsilon_{\delta}^{N_{k}}>0$ então $N_{k}=N_{\max }, N_{k}^{\min }=N_{\max }$ e volte ao Passo 2 .

3.2. Se $\varepsilon_{\delta}^{N_{k}}=0$ então $N_{k}=N_{k}+1, N_{k}^{\text {min }}=N_{k}^{\text {min }}+1$ e volte ao Passo 2.

Passo 4. Encontre $d_{k}$ tal que $d_{k}^{T} \nabla \hat{f}_{N_{k}}\left(x_{k}\right)<0$.

Passo 5. (Condição de Armijo) Encontre $\alpha_{k}$ tal que:

$$
\hat{f}_{N_{k}}\left(x_{k}+\alpha_{k} d_{k}\right) \leq \hat{f}_{N_{k}}\left(x_{k}\right)+\eta \alpha_{k} d_{k}^{T} \nabla \hat{f}_{N_{k}}\left(x_{k}\right) .
$$

Passo 6. $x_{k+1}=x_{k}+\alpha_{k} d_{k}$ e calcule $d m_{k}$ usando (2.18).

Passo 7. Determine o candidato a tamanho amostral $N_{k}^{+}$usando o Algoritmo 2.2.3.

Passo 8. Determine o tamanho amostral $N_{k+1}$ usando o Algoritmo 2.2.4.

Passo 9. Determine a cota inferior do tamanho amostral $N_{k+1}^{m i n}$.

Passo 10. $k \leftarrow k+1$ e volte ao Passo 2 até que $\left\|\nabla \hat{f}_{N_{k}}\left(x_{k}\right)\right\|=0$ e $N_{k}=N_{\max }$.

Algoritmo 2.2.3. CAndidato a tamanho amostral

Entrada: Sejam $d m_{k}, N_{k}^{\text {min }}, \varepsilon_{\delta}^{N_{k}}\left(x_{k}\right)$ e $\nu_{1} \in(0,1)$ dados.

Passo 1. Se $d m_{k}=\varepsilon_{\delta}^{N_{k}}\left(x_{k}\right)$ então $N_{k}^{+}=N_{k}$.

Passo 2. Se $d m_{k}>\varepsilon_{\delta}^{N_{k}}\left(x_{k}\right)$ então $N_{k}^{+} \leftarrow N_{k}$. Enquanto $d m_{k}>\varepsilon_{\delta}^{N_{k}^{+}}\left(x_{k}\right)$ e $N_{k}^{+}>N_{k}^{\text {min }}$ faça $N_{k}^{+}=N_{k}^{+}-1$.

Passo 3. Se $d m_{k}<\varepsilon_{\delta}^{N_{k}}\left(x_{k}\right)$

3.1. Se $d m_{k} \geq \nu_{1} \varepsilon_{\delta}^{N_{k}}\left(x_{k}\right)$ então $N_{k}^{+} \leftarrow N_{k}$. Enquanto $d m_{k}<\varepsilon_{\delta}^{N_{k}^{+}}\left(x_{k}\right)$ e $N_{k}^{+}<N_{\max }$ faça $N_{k}^{+}=N_{k}^{+}+1$.

3.2. Se $d m_{k}<\nu_{1} \varepsilon_{\delta}^{N_{k}}\left(x_{k}\right)$ então $N_{k}^{+}=N_{\max }$. 


\section{Algoritmo 2.2.4. DETERMINAÇÃO DO CANDIDADO A TAMANHO AMOSTRAL}

Entrada: Sejam $N_{k}^{+}, N_{k}, x_{k}, x_{k+1}, \eta_{0}<1$ dados.

Passo 1. Determine $N_{k+1}$

1.1. Se $N_{k}^{+} \geq N_{k}$ então $N_{k+1}=N_{k}^{+}$.

1.2. Se $N_{k}^{+}<N_{k}$ calcule

$$
\rho_{k}=\frac{\hat{f}_{N_{k}^{+}}\left(x_{k}\right)-\hat{f}_{N_{k}^{+}}\left(x_{k+1}\right)}{\hat{f}_{N_{k}}\left(x_{k}\right)-\hat{f}_{N_{k}}\left(x_{k+1}\right)}
$$

Se $\rho_{k} \geq \eta_{0}$ então $N_{k+1}=N_{k}^{+}$senão $N_{k+1}=N_{k}$.

Passo 2. Atualize $N_{k}^{\text {min }}$

2.1. Se $N_{k+1} \leq N_{k}$ então $N_{k+1}^{\min }=N_{k}^{\min }$.

2.2. Se $N_{k+1}>N_{k}$ então

2.2.1. Se o tamanho $N_{k+1}$ nunca foi usado, então $N_{k+1}^{\min }=N_{k}^{\min }$.

2.2.2. Se alcançou-se suficiente decrescimento em $\hat{f}_{N_{k+1}}$ desde a última vez que $N_{k+1}$ foi usada, então $N_{k+1}^{\min }=N_{k}^{\min }$.

2.2.3. Se não alcançou-se suficiente decrescimento em $\hat{f}_{N_{k+1}}$ desde a última vez que $N_{k+1}$ foi usada, então $N_{k+1}^{\min }=N_{k+1}$.

No Algoritmo 2.2.4 no Passo 2 se diz que "não alcançou-se suficiente decrescimento em $\hat{f}_{N_{k+1}}$ " se, dadas as constantes $\gamma_{3}, \nu_{1} \in(0,1)$ é satisfeita a seguinte desigualdade:

$$
\hat{f}_{N_{k+1}}\left(x_{h_{(k)}}\right)-\hat{f}_{N_{k+1}}\left(x_{k+1}\right)<\gamma_{3} \nu_{1}(k+1-h(k)) \varepsilon_{\delta}^{N_{k+1}}\left(x_{k+1}\right)
$$

onde $h(k)$ é a iteração na qual começou-se a usar o tamanho $N_{k+1}$ por última vez.

As seguintes suposições a enunciar são necessárias para garantir que a convergência do Algoritmo 2.2.2 seja alcançada.

Suposição 2.2.1. Existe uma constante $M_{1}>0$ tal que para todo $\xi$, $x$ temos que $\left\|\nabla_{x} F(x, \xi)\right\| \leq$ $M_{1}$.

Suposição 2.2.2. Existem constantes $M_{F}, M_{F F}$ tal que para todo $\xi, x$ temos que $M_{F} \leq F(x, \xi) \leq$ $M_{F F}$.

Suposição 2.2.3. A sequência de direções $d_{k}$ geradas no Passo 4 do Algoritmo 2.2.2 é acotada e satisfaz que se $\lim _{k \in K} d_{k}^{T} \nabla \hat{f}_{N_{k}}\left(x_{k}\right)=0$ então $\lim _{k \in K} \nabla \hat{f}_{N_{k}}\left(x_{k}\right)=0$ para qualquer subconjunto de iterações $K$.

As Suposições 2.2.1 e 2.2.2 garantem que o Teorema de Intercambiabilidade entre o gradiente e a integral (i.e. Teorema 2.1.3) é satisfeito, e portanto, $\nabla \mathbb{E}(f(x))=\mathbb{E}(\nabla f(x))$. Assim, juntando esse fato com a Lei de grandes números, obtemos que $\lim _{N \rightarrow \infty} \nabla \hat{f}_{N}(x)=\nabla f(x)$. A Suposição 2.2.3 permite definir no algoritmo qualquer direção $d_{k}$ de descida.

O seguinte lema permite garantir que o tamanho amostral $N_{\max }$ é alcançado, e que a partir de certa iteração, esse $N_{k}=N_{\max }$ é mantido. Este lema é esencial para a demostração do Teorema 2.2.2 que enuncia a convergência do Algoritmo 2.2 .2 a algum ponto estacionário da função $\hat{f}_{N_{\max }}$. Os detalhes das demostrações podem ser encontrados em [KK13]. No texto abaixo abordaremos apenas algumas ideias principais.

Lema 2.2.1. [KK13] Suponha que são satisfeitas as Suposições 2.2.1 e 2.2.2. Suponha também que existe uma constante positiva $\omega$ e $k_{1} \in \mathbb{N}$ tais que $\varepsilon_{\delta}^{N_{k}}\left(x_{k}\right) \geq \omega$ para todo $k \geq k_{1}$. Logo, o Algoritmo 2.2.2 termina em um número finito de iterações com $N_{k}=N_{\text {max }}$ ou existe $k_{2} \in \mathbb{N}$ tal que para cada $k \geq k_{2}$ o tamanho amostral é máximo, i.e. $N_{k}=N_{\max }$. 
Demonstração.

Como a condição de parada do Algoritmo 2.2.2 é $\left\|\nabla \hat{f}_{N_{\max }}\left(x_{k}\right)\right\|=0$, o foco da demostração é provar que o algoritmo faz um número finito de iterações. Especificamente, mostraremos que $N_{k}$ não fica "encalhado" em tamanhos menores a $N_{\max }$ e que a sequência não oscila, isto é, existe $\bar{k} \in \mathbb{N}$ tal que para todo $k \geq \bar{k}$ temos que $N_{k}=N_{\max }$.

- $N_{\max }$ é alcançado:

Suponha que existe $n_{2}>n_{1}$ tal que para todo $k \geq n_{2}$ temos que $N_{k}=N^{\prime}<N_{\max }$. Logo, pela condição de Armijo temos que:

$$
\hat{f}_{N^{\prime}}\left(x_{k+1}\right) \leq \hat{f}_{N^{\prime}}\left(x_{k}\right)+\eta \alpha_{k} \nabla \hat{f}_{N^{\prime}}\left(x_{k}\right)^{T} d_{k} .
$$

Assim, para cada $s \in\{0,1,2, \ldots\}$ temos

$$
\begin{aligned}
\hat{f}_{N^{\prime}}\left(x_{n_{2}+s}\right) & \leq \hat{f}_{N^{\prime}}\left(x_{n_{2}+s-1}\right)+\eta \alpha_{n_{2}+s-1} \nabla \hat{f}_{N^{\prime}}\left(x_{n_{2}+s-1}\right)^{T} d_{n_{2}+s-1} \\
& \leq \hat{f}_{N^{\prime}}\left(x_{n_{2}}\right)+\eta \sum_{j=0}^{s-1} \alpha_{n_{2}+j} \nabla \hat{f}_{N^{\prime}}\left(x_{n_{2}+j}\right)^{T} d_{n_{2}+j} .
\end{aligned}
$$

Logo, pela Suposição 2.2.2 obtemos

$$
-\eta \sum_{j=0}^{s-1} \alpha_{n_{2}+j} \nabla \hat{f}_{N^{\prime}}\left(x_{n_{2}+j}\right)^{T} d_{n_{2}+j} \leq \hat{f}_{N^{\prime}}\left(x_{n_{2}}\right)-\hat{f}_{N^{\prime}}\left(x_{n_{2}+s}\right) \leq \hat{f}_{N^{\prime}}\left(x_{n_{2}}\right)-M_{F}
$$

e, tomando o limite, temos que

$$
0 \leq \sum_{j=0}^{\infty}-\alpha_{n_{2}+j} \nabla \hat{f}_{N^{\prime}}\left(x_{n_{2}+j}\right)^{T} d_{n_{2}+j} \leq \frac{\hat{f}_{N^{\prime}}\left(x_{n_{2}}\right)-M_{F}}{\eta}:=C .
$$

Como a serie em (2.20) é convergente vale que

$$
\lim _{j \rightarrow \infty}-\alpha_{n_{2}+j} \nabla \hat{f}_{N^{\prime}}\left(x_{n_{2}+j}\right)^{T} d_{n_{2}+j}=0 .
$$

Agora, observando o Algoritmo 2.2 .3 e usando a suposição de que $\varepsilon_{\delta}^{N_{k}}\left(x_{k}\right) \geq \omega$ temos que unicamente o caso $d m_{k}<\nu_{1} \varepsilon_{\delta}^{N_{k}}$ (Passo 3) não pode acontecer pois levaria a $N_{k+1} \geq N_{k}^{+}=$ $N_{\max }>N^{\prime}$, porem, para todo $k>n_{2},-\alpha_{k} \nabla \hat{f}_{N^{\prime}}\left(x_{k}\right)^{T} d_{k} \geq \nu_{1} \omega$. Assim,

$$
\liminf _{k \rightarrow \infty}-\alpha_{k} \nabla \hat{f}_{N^{\prime}}\left(x_{k}\right)^{T} d_{k} \geq \nu_{1} \omega>0
$$

que contradiz (2.21). Portanto, demonstrou-se que a sequência dos tamanhos amostrais $N_{k}$ não fica encalhado em tamanhos menores a $N_{\max }$.

- A sequência de $N_{k}$ não oscila:

Suponha o contrário, quer dizer, para todo $\bar{k}$ existe $k \geq \bar{k}$ tal que $N_{k}<N_{\max }$ implicando assim que $N_{k}^{\min }<N_{\max }$. Assim, a atualização no Passo 2.2.3 do Algoritmo 2.2.4 ocorre um número finito de vezes. Logo, as outras 3 possibilidades (Passos 2.1, 2.2.1, 2.2.2, onde $N_{k+1}^{\min }=N_{k}^{\mathrm{min}}$ ) ocorrem um número infinito de vezes. Portanto, existe $r_{1}$ tal que para todo $k \geq r_{1}$ temos que acontece alguma das seguintes opções:

1. $N_{k+1} \leq N_{k}$.

2. $N_{k+1}>N_{k}$ e não temos usado o tamanho amostral $N_{k+1}$ antes.

3. $N_{k+1}>N_{k}$ e se tem suficiente decrescimento em $\hat{f}_{N_{k+1}}$ desde a última vez que $N_{k+1}$ foi usada. 
Seja $\bar{N}$ o tamanho máximo que é usado infinitas iterações, e seja $\bar{K}_{0}$ o conjunto de iterações onde $N_{k}$ cambia a $\bar{N}$. Assim, seja $r_{2}$ a iteração tal que para todo $k \in \bar{K}_{0}, k \geq r_{2} N_{k}$ é aumentado a $\bar{N}$.

Defina $r=\max \left\{r_{1}, r_{2}\right\}$ e $\bar{K}=\bar{K}_{0} \cap\{r, r+1, \ldots\}$. Como $\bar{K}$ possui os elementos de $\bar{K}_{0}$, então, a opção de $N_{k+1} \leq N_{k}$ não acontece. Logo, se tiramos de $\bar{K}$ a iteração onde $N_{k+1}$ foi usado pela primeira vez, exclui-se a segunda opção anterior também. Assim, para todo $k \in \bar{K} \cap\left\{n_{1}, n_{1}+1, \ldots\right\}$ temos que

$$
\begin{aligned}
\hat{f}_{\bar{N}}\left(x_{h(k)}\right)-\hat{f}_{\bar{N}}\left(x_{k+1}\right) & \geq \gamma_{3} \nu_{1}(k+1-h(k)) \varepsilon_{\delta}^{\bar{N}}\left(x_{k+1}\right) \\
& \geq \gamma_{3} \nu_{1} \varepsilon_{\delta}^{\bar{N}}\left(x_{k+1}\right) \\
& \geq \gamma_{3} \nu_{1} \omega>0 .
\end{aligned}
$$

O que afirma que estamos diminuindo $\hat{f}_{\bar{N}}$ por uma constante positiva infinitas vezes. Mas, pela Suposição 2.2.2 (que implica que $\hat{f}_{N}(x)$ é acotada superior e inferiormente) chegamos a uma contradição.

Portanto, a sequência de $N_{k}$ não oscila e $N_{k}^{\min }=N_{\max }$ para algum $k$. Finalmente, juntando com a primeira parte da demonstração obtemos que existe uma iteração $k_{1} \in \mathbb{N}$ tal que para cada $k \geq k_{1}$ temos que $N_{k}=N_{\text {max }}$.

Teorema 2.2.2. [KK13] Suponha que são satisfeitas Suposições 2.2.1-2.2.3. Suponha também que existe uma constante positiva $\omega$ e $k_{1} \in \mathbb{N}$ tal que $\varepsilon_{\delta}^{N_{k}}\left(x_{k}\right) \geq \omega$ para todo $k \geq k_{1}$ e que a sequência $\left\{x_{k}\right\}$ gerada pelo Algoritmo 2.2.2 é limitada. Então, o Algoritmo 2.2.2 termina depois de um número finito de iterações num ponto estacionário da função $\hat{f}_{N_{\max }}$ ou todo ponto de acumulação da sequência $\left\{x_{k}\right\}$ é um ponto estacionário de $\hat{f}_{N_{\max }}$.

Demonstração.

A demostração detalhada pode ser encontrada em [KK13]. A ideia da prova é estudar o caso em que a cada iteração se tem uma direção de descida, gerando assim infinitas iterações. O Lema 2.2.1 garante a existência de $k_{1} \in \mathbb{N}$ tal que para todo $k \geq k_{1}$ temos que $N_{k}=N_{\max }$. Queremos provar que existe um conjunto $K^{\prime} \subseteq \mathbb{N}$ tal que $\lim _{k \in K^{\prime}} x_{k}=x^{*}$ e $\nabla \hat{f}_{N_{\max }}\left(x^{*}\right)=\lim _{k \in K^{\prime}} \nabla \hat{f}_{N_{\max }}\left(x_{k}\right)=0$.

Pela equação (2.21), temos que

$$
\lim _{k \rightarrow \infty} \alpha_{k} \nabla \hat{f}_{N_{\max }}\left(x_{k}\right)^{T} d_{k}=0 .
$$

Logo, seja $x^{*}$ um ponto de acumulação da sequência $\left\{x_{k}\right\}_{k \in \mathbb{N}}$, isto implica que existe $K \subseteq \mathbb{N}$ tal que $\lim _{k \in K} x_{k}=x^{*}$. Temos assim as seguintes opções:

- A sequência dos passos $\left\{\alpha_{k}\right\}_{k \in K}$ é limitada inferiormente, então, (2.22) implica que

$$
\lim _{k \in K} \nabla \hat{f}_{N_{\max }}\left(x_{k}\right)^{T} d_{k}=0 .
$$

e portanto, pela Suposição 2.2.3 obtemos que

$$
\nabla \hat{f}_{N_{\max }}\left(x^{*}\right)=\lim _{k \in K} \nabla \hat{f}_{N_{\max }}\left(x_{k}\right)=0 .
$$

- Existe $K_{1} \subseteq K$ tal que $\lim _{k \in K_{1}} \alpha_{k}=0$ isto garante a existência de um subconjunto $K_{2} \subseteq K_{1}$ tal que para todo $k \in K_{2}$ existe $\alpha_{k}^{\prime}$ tal que $\alpha_{k}=\beta \alpha_{k}^{\prime} \mathrm{e}$

$$
\hat{f}_{N_{\max }}\left(x_{k}+\alpha_{k}^{\prime} d_{k}\right)>\hat{f}_{N_{\max }}\left(x_{k}\right)+\eta \alpha_{k}^{\prime} \nabla \hat{f}_{N_{\max }}\left(x_{k}\right)^{T} d_{k} .
$$

Pelo teorema de Valor médio temos que a expressão anterior pode ser reescrita como

$$
d_{k}^{T} \nabla \hat{f}_{N_{\max }}\left(x_{k}+t_{k} \alpha_{k}^{\prime} d_{k}\right)>\eta \nabla \hat{f}_{N_{\max }}\left(x_{k}\right)^{T} d_{k}
$$


para algum $t_{k} \in[0,1]$. Depois, como as direções $d_{k}$ estão acotadas, existe $d^{*}$ e $K_{3} \subseteq K_{2}$ tal que $\lim _{k \in K_{3}} d_{k}=d^{*}$. Assim,

$$
\nabla \hat{f}_{N_{\max }}\left(x^{*}\right)^{T} d^{*}>\eta \nabla \hat{f}_{N_{\max }}\left(x^{*}\right)^{T} d^{*}
$$

Logo, como $\eta \in(0,1)$ e $\nabla \hat{f}_{N_{\max }}\left(x_{k}\right)^{T} d_{k}<0$ para todo $k$, juntando com a Suposição 2.2 .3 obtemos que:

$$
\lim _{k \in K_{3}} \nabla \hat{f}_{N_{\max }}\left(x_{k}\right)^{T} d_{k}=\nabla \hat{f}_{N_{\max }}\left(x^{*}\right)^{T} d^{*}=0
$$

e assim,

$$
\lim _{k \in K_{3}} \nabla \hat{f}_{N_{\max }}\left(x_{k}\right)=0
$$

\subsection{Métodos de Redução da variância}

Nas seções anteriores se estudaram propostas para a resolução do problema (2.7). Uma possível forma de ver o algoritmo de Stochastic Gradient Descent é, como nas aplicações de Aprendizado de Máquina, dados $N$ pontos $\xi_{1}, \ldots, \xi_{N}$ i.i.d. gerar a aproximação de Monte Carlo, e em cada iteração tomar $N_{k}=1$ para todo $k$, isto é, aproximar o gradiente de $\hat{f}_{N}(x)$ com o gradiente de $\hat{f}_{1}(x)=F\left(x, \xi_{k}\right)$.

Monte Carlo y Stochastic Approximation geram aproximações do valor esperado, mas é importante estudar a eficiência dessa estimação. Desde o ponto de vista estatístico, existem dois fatores importantes no momento de escolher os melhores estimadores: consistência e variância. O primeiro fator verifica se $\mathbb{E}(\hat{f}(x))=f(x)$, e o segundo se relaciona com o intervalo de confiança da estimação feita, isto é, se espera que a variância seja pequena para que o intervalo de confiança obtido seja menor. Nos dois enfoques vistos, com as suposições feitas, se geram estimadores sem viés, assim, para escolher o melhor (no sentido estatístico) a variância é de suma importância.

Os métodos de Redução da variância [AG07, KKK10, Rip87] foram motivados pelo fato que o desvio padrão da diferencia entre a estimação de Monte Carlo e o verdadeiro valor esperado é da ordem de $1 / \sqrt{N}$. Matematicamente, sejam $x_{1}, \ldots, x_{N} N$ variáveis aleatórias i.i.d. de $X$ definidas num espaço de probabilidades $(\Omega, \mathcal{F}, P)$ e seja $\bar{x}_{N}:=\frac{1}{N} \sum_{i=1}^{N} x_{i}$ o estimador de Monte Carlo para $\mu=\mathbb{E}(X)$, então,

$$
\operatorname{Var}\left(\bar{x}_{N}-\mu\right)=\operatorname{Var}\left(\bar{x}_{N}\right)=\frac{1}{N^{2}} \sum_{i=1}^{N} \operatorname{Var}\left(x_{i}\right)=\frac{\operatorname{Var}(X)}{N} .
$$

Portanto, para gerar pequenas melhoras na precição da estimação, se devem obter amostras muito maiores. Existem diferentes métodos de Redução de variância, entre eles: Control Variates, Importance Sampling, Antithetic Variates e Conditioning. Cada um deles podem ser analisados a fundo em [AG07, KKK10, Rip87]. Especificamente neste trabalho estudaremos Control Variates.

Suponha que se quer estimar $\mu=\mathbb{E}(Y)$, onde $Y=h(x)$. Gostaríamos de encontrar uma variável $Z$ tal que $\mathbb{E}(Z)=\mu$ e $\operatorname{Var}(Z)<\operatorname{Var}(Y)$. Seja $W=g(x)$ uma função tal que se conhece $\mathbb{E}(W)=\mu_{W}$. Definamos $Z=\alpha(Y-W)+\mathbb{E}(W), \operatorname{com} \alpha \in[0,1]$, temos que,

$$
\begin{aligned}
\mathbb{E}(Z) & =\mathbb{E}(\alpha(Y-W)+\mathbb{E}(W)) \\
& =\alpha \mathbb{E}(Y)+(1-\alpha) \mathbb{E}(W) \\
\operatorname{Var}(Z) & =\operatorname{Var}(\alpha(Y-W)+\mathbb{E}(W)) \\
& =\alpha^{2} \operatorname{Var}(Y-W) \\
& =\alpha^{2}(\operatorname{Var}(Y)+\operatorname{Var}(W)-2 \operatorname{Cov}(Y, W))
\end{aligned}
$$


Se considera-se $\alpha=1$ obtemos que $\mathbb{E}(Z)=\mathbb{E}(Y)$. A ideia do método Control Variate é encontrar uma variável $W$ tal que $2 \operatorname{Cov}(Y, W)-\operatorname{Var}(W)$ seja o suficientemente grande que permita obter que $\operatorname{Var}(Z)<\operatorname{Var}(Y)$.

A seguir se apresentam dois algoritmos que tentam melhorar a estimação do gradiente por meio de métodos de redução da variância usando Control Variates.

\subsubsection{Stochastic Variance Reduced Gradient}

Inspirados na teoria de Variance Reduction - Control Variates, Jhonson e Zhang [JZ13] propõem um algoritmo que busca diminuir a variância da estimação do gradiente do Algoritmo 2.1.2 (Stochastic Gradient Descent).

Especificamente, voltando ao problema (2.12), se quer encontrar um algoritmo que consiga a solução de

$$
\min _{x \in \mathbb{R}^{d}}\left\{\hat{f}_{N}(x):=\frac{1}{N} \sum_{i=1}^{N} F\left(x, \xi_{i}\right)\right\}
$$

Portanto, continuando com o análise de Control Variate, seja $Y=\nabla F\left(x, \xi_{j}\right)$ a estimação do gradiente de $\hat{f}_{N}(x)$ para o Algoritmo 2.1.2 (Stochastic Gradient Descent). Definamos $W=\nabla F\left(\tilde{x}, \xi_{j}\right.$ ) com $\tilde{x}$ uma atualização do minimizador de $\hat{f}_{N}(x)$ passadas $m$ iterações. Assim, considerando $Z=$ $Y-W+\mathbb{E}(W)$ obtemos que $Z$ é um estimador consistente de $Y$ tal que $\operatorname{Var}(Z)<\operatorname{Var}(Y)$.

O Algoritmo 2.3.1 apresenta uma modificação do Algoritmo 2.1.2 que diminui a variância da estimação do gradiente.

Algoritmo 2.3.1. Stochastic Variance Reduced Gradient

Entrada: $m$ : parâmetro de frequência de atualização;

$\eta$ : learning rate.

Passo 1. Inicialize $\tilde{x}_{0}, s \leftarrow 1$

Passo 2. $\tilde{x}=\tilde{x}_{s-1}$

Passo 3. $\tilde{\mu}=\frac{1}{N} \sum_{i=1}^{N} \nabla F\left(\tilde{x}, \xi_{i}\right)$

Passo 4. $x_{0}=\tilde{x}$

Passo 5. Para $k=0, \ldots, m-1$

5.1. Escolha aleatoriamente $j \in\{1, \ldots, N\}$

5.2. $x_{k+1}=x_{k}-\eta\left(\nabla F\left(x_{k}, \xi_{j}\right)-\nabla F\left(\tilde{x}, \xi_{j}\right)+\tilde{\mu}\right)$

Passo 6. (Opção I) $\tilde{x}_{s}=x_{m}$

Passo 7. (Opção II) $\tilde{x}_{s}=x_{t}$ para $t \in\{0, \ldots, m\}$ aleatório.

Passo 8. $s \leftarrow s+1$

Passo 9. Volte ao Passo 2 até satisfazer condição de parada.

Agora, suponha que $F$ é suave e convexa e o gradiente $\nabla F$ é Lipschitz com parâmetro $L$, então temos que se satisfazem as seguintes proposições:

Proposição 2.3.1. Para todo $x, x^{\prime}, \xi_{i}$ temos que $F\left(x, \xi_{i}\right)-F\left(x^{\prime}, \xi_{i}\right)-0.5 L\left\|x-x^{\prime}\right\|^{2} \leq \nabla F\left(x^{\prime}, \xi_{i}\right)^{T}(x-$ $\left.x^{\prime}\right)$. 
Demonstração. Consideremos a expansão de Taylor de ordem 0:

$$
\begin{aligned}
F\left(x^{\prime}+d, \xi_{i}\right) & =F\left(x^{\prime}, \xi_{i}\right)+\int_{0}^{1} \nabla F\left(x^{\prime}+\varepsilon d, \xi_{i}\right)^{T} d \partial \varepsilon \\
& =F\left(x^{\prime}, \xi_{i}\right)+\nabla F\left(x^{\prime}, \xi_{i}\right)^{T} d-\nabla F\left(x^{\prime}, \xi_{i}\right)^{T} d+\int_{0}^{1} \nabla F\left(x^{\prime}+\varepsilon d, \xi_{i}\right)^{T} d \partial \varepsilon \\
& =F\left(x^{\prime}, \xi_{i}\right)+\nabla F\left(x^{\prime}, \xi_{i}\right)^{T} d+\int_{0}^{1}\left(\nabla F\left(x^{\prime}+\varepsilon d, \xi_{i}\right)-\nabla F\left(x^{\prime}, \xi_{i}\right)\right)^{T} d \partial \varepsilon \\
& \leq F\left(x^{\prime}, \xi_{i}\right)+\nabla F\left(x^{\prime}, \xi_{i}\right)^{T} d+\int_{0}^{1}\left\|\nabla F\left(x^{\prime}+\varepsilon d, \xi_{i}\right)-\nabla F\left(x^{\prime}, \xi_{i}\right)\right\|\|d\| \partial \varepsilon .
\end{aligned}
$$

Logo, como $\nabla F$ é Lipschitz temos que:

$$
\begin{aligned}
F\left(x^{\prime}+d, \xi_{i}\right) & \leq F\left(x^{\prime}, \xi_{i}\right)+\nabla F\left(x^{\prime}, \xi_{i}\right)^{T} d+\|d\| \int_{0}^{1} L\left\|x^{\prime}+\varepsilon d-x^{\prime}\right\| \partial \varepsilon \\
& =F\left(x^{\prime}, \xi_{i}\right)+\nabla F\left(x^{\prime}, \xi_{i}\right)^{T} d+L\|d\|^{2} \int_{0}^{1} \varepsilon \partial \varepsilon \\
& =F\left(x^{\prime}, \xi_{i}\right)+\nabla F\left(x^{\prime}, \xi_{i}\right)^{T} d+0.5 L\|d\|^{2} .
\end{aligned}
$$

Assim, seja $d=x-x^{\prime}$, obtemos que:

$$
F\left(x^{\prime}, \xi_{i}\right) \leq F\left(x^{\prime}, \xi_{i}\right)+\nabla F\left(x^{\prime}, \xi_{i}\right)^{T}\left(x-x^{\prime}\right)+0.5 L\left\|x-x^{\prime}\right\|^{2} .
$$

Proposição 2.3.2. se $f(x)$ é estritamente convexa, então para todo $x, x^{\prime}, \xi_{i}$ temos que $f(x)-$ $f\left(x^{\prime}\right)-0.5 \gamma\left\|x-x^{\prime}\right\|^{2} \geq \nabla f\left(x^{\prime}\right)^{T}\left(x-x^{\prime}\right)$ com $L \geq \gamma \geq 0$.

Proposição 2.3.3. Para todo $x, x^{\prime}, \xi_{i}$ temos que

$$
\frac{1}{n} \sum_{i=1}^{n}\left\|\nabla F\left(x, \xi_{i}\right)-\nabla F\left(x^{\prime}, \xi_{i}\right)\right\|^{2} \leq 2 L\left[\frac{1}{n} \sum_{i=1}^{n}\left(F\left(x, \xi_{i}\right)-F\left(x^{\prime}, \xi_{i}\right)\right)-\frac{1}{n} \sum_{i=1}^{n} \nabla F\left(x^{\prime}, \xi_{i}\right)^{T}\left(x-x^{\prime}\right)\right] .
$$

Demonstração. Seja

$$
g_{i}(x)=F\left(x, \xi_{i}\right)-F\left(x^{\prime}, \xi_{i}\right)-\nabla F\left(x^{\prime}, \xi_{i}\right)^{T}\left(x-x^{\prime}\right) .
$$

Temos que $x^{\prime}$ é o minimizador de $g_{i}(x)$, pois

$$
\nabla g_{i}\left(x^{\prime}\right)=\nabla F\left(x^{\prime}, \xi_{i}\right)-\nabla F\left(x^{\prime}, \xi_{i}\right)=0 .
$$

Assim, aplicando a propriedade de minimizador e a suavidade e convexidade de $F$ demonstrada na Proposição 2.3.1 temos

$$
\begin{aligned}
0=g_{i}\left(x^{\prime}\right) & \leq \min _{\eta}\left(g_{i}\left(x-\eta \nabla g_{i}(x)\right)\right) \\
& \left.\leq \min _{\eta}\left(g_{i}(x)-\eta\left\|\nabla g_{i}(x)\right\|_{2}^{2}+0.5 L \eta^{2}\left\|\nabla g_{i}(x)\right\|_{2}^{2}\right)\right) \\
& =g_{i}(x)-\left\|\nabla g_{i}(x)\right\|_{2}^{2} \min _{\eta}\left(\eta-\frac{1}{2} L \eta^{2}\right) .
\end{aligned}
$$

Logo, $\min _{\eta}\left(\eta-\frac{1}{2} L \eta^{2}\right)=\frac{1}{2 L}$ pois $\frac{\partial}{\partial \eta}\left(\eta-\frac{1}{2} L \eta^{2}\right)=1-2 L \eta=0 \leftrightarrow \eta=\frac{1}{2 L}$. Voltando em (2.23) e substituindo $g_{i}(x)$ obtemos 


$$
\begin{aligned}
0 & \leq g_{i}(x)-\frac{1}{2 L}\left\|\nabla g_{i}(x)\right\|_{2}^{2} \\
& =F\left(x, \xi_{i}\right)-F\left(x^{\prime}, \xi_{i}\right)-\nabla F\left(x^{\prime}, \xi_{i}\right)^{T}\left(x-x^{\prime}\right)-\frac{1}{2 L}\left\|\nabla F\left(x, \xi_{i}\right)-\nabla F\left(x^{\prime}, \xi_{i}\right)\right\|^{2} .
\end{aligned}
$$

Reescrevendo,

$$
\left\|\nabla F\left(x, \xi_{i}\right)-\nabla F\left(x^{\prime}, \xi_{i}\right)\right\|^{2} \leq 2 L\left(F\left(x, \xi_{i}\right)-F\left(x^{\prime}, \xi_{i}\right)-\nabla F\left(x^{\prime}, \xi_{i}\right)^{T}\left(x-x^{\prime}\right)\right) .
$$

Somando por $i=1, \ldots, n$ e usando que $f(x)=\sum_{i=1}^{n} F\left(x, \xi_{i}\right)$ temos que

$\frac{1}{n} \sum_{i=1}^{n}\left\|\nabla F\left(x, \xi_{i}\right)-\nabla F\left(x^{\prime}, \xi_{i}\right)\right\|^{2} \leq 2 L\left[\frac{1}{n} \sum_{i=1}^{n}\left(F\left(x, \xi_{i}\right)-F\left(x^{\prime}, \xi_{i}\right)\right)-\frac{1}{n} \sum_{i=1}^{n} \nabla F\left(x^{\prime}, \xi_{i}\right)^{T}\left(x-x^{\prime}\right)\right]$.

O seguinte teorema estipula a convergência geométrica (em sentido de média quadrada) do Algoritmo 2.3.1.

Teorema 2.3.1. [JZ13] Considere o Algoritmo 2.3.1 com a opção II. Suponha que $F\left(x, \xi_{i}\right)$ é convexa e suave para todo $x$ e $\xi_{i}$ e que $f(x)$ é fortemente convexa com parâmetro $\gamma>0$. Seja $x^{*}=\underset{x \in \mathbb{R}^{d}}{\operatorname{argmin}} f(x)$. Suponha que $m$ é o suficientemente grande tal que

$$
\alpha=\frac{1}{\gamma \eta(1-2 L \eta) m}+\frac{2 L \eta}{1-2 L \eta}<1
$$

então temos convergência geométrica em valor esperado para o Algoritmo 2.3.1:

$$
\mathbb{E}\left(f\left(\tilde{x}_{s}\right)-f\left(x^{*}\right)\right) \leq \alpha^{s}\left(f\left(\tilde{x}_{0}\right)-f\left(x^{*}\right)\right) .
$$

Demonstração. Seja, $v_{t}=\nabla F\left(x_{t-1}, \xi_{i_{t}}\right)-\nabla F\left(\tilde{x}, \xi_{i_{t}}\right)+\tilde{\mu}$.

$$
\begin{aligned}
\mathbb{E}\left(\left\|v_{t}\right\|_{2}^{2} \mid x_{t-1}\right) & =\mathbb{E}\left(\left\|\nabla F\left(x_{t-1}, \xi_{i_{t}}\right)-\nabla F\left(\tilde{x}, \xi_{i_{t}}\right)+\tilde{\mu}\right\|_{2}^{2} \mid x_{t-1}\right) \\
& =\mathbb{E}\left(\left\|\nabla F\left(x_{t-1}, \xi_{i_{t}}\right)-\nabla F\left(x^{*}, \xi_{i_{t}}\right)+\nabla F\left(x^{*}, \xi_{i_{t}}\right)-\nabla F\left(\tilde{x}, \xi_{i_{t}}\right)+\tilde{\mu}\right\|_{2}^{2} \mid x_{t-1}\right) .
\end{aligned}
$$

Pela desigualdade de Cauchy-Schuarz temos que $\|a+b\|_{2}^{2} \leq 2\|a\|_{2}^{2}+2\|b\|_{2}^{2}$ e considerando que

$$
\begin{aligned}
\mathbb{E}\left(\nabla F\left(\tilde{x}, \xi_{i_{t}}\right)-\nabla F\left(x^{*}, \xi_{i_{t}}\right) \mid x_{t-1}\right) & =\mathbb{E}\left(\nabla F\left(\tilde{x}, \xi_{i_{t}}\right) \mid x_{t-1}\right)-\mathbb{E}\left(\nabla F\left(x^{*}, \xi_{i_{t}}\right) \mid x_{t-1}\right) \\
& =\nabla f(\tilde{x})-\nabla f\left(x^{*}\right)=\nabla f(\tilde{x})=\tilde{\mu},
\end{aligned}
$$

substituimos em (2.24) e obtemos que

$$
\begin{aligned}
\mathbb{E}\left(\left\|v_{t}\right\|_{2}^{2} \mid x_{t-1}\right) \leq & \mathbb{E}\left(2\left\|\nabla F\left(x_{t-1}, \xi_{i_{t}}\right)-\nabla F\left(x^{*}, \xi_{i_{t}}\right)\right\|_{2}^{2}+2 \|-\left(\nabla F\left(\tilde{x}, \xi_{i_{t}}\right)-\nabla F\left(x^{*}, \xi_{i_{t}}\right)-\tilde{\mu} \|_{2}^{2} \mid x_{t-1}\right)\right. \\
= & 2 \mathbb{E}\left(\left\|\nabla F\left(x_{t-1}, \xi_{i_{t}}\right)-\nabla F\left(x^{*}, \xi_{i_{t}}\right)\right\|_{2}^{2} \mid x_{t-1}\right)+ \\
& \quad+2 \mathbb{E}\left(\|\left(\nabla F\left(\tilde{x}, \xi_{i_{t}}\right)-\nabla F\left(x^{*}, \xi_{i_{t}}\right)-\tilde{\mu} \|_{2}^{2} \mid x_{t-1}\right)\right. \\
= & 2 \mathbb{E}\left(\left\|\nabla F\left(x_{t-1}, \xi_{i_{t}}\right)-\nabla F\left(x^{*}, \xi_{i_{t}}\right)\right\|_{2}^{2} \mid x_{t-1}\right)+ \\
& \quad+2 \mathbb{E}\left(\left\|\left[\nabla F\left(\tilde{x}, \xi_{i_{t}}\right)-\nabla F\left(x^{*}, \xi_{i_{t}}\right)\right]-\mathbb{E}\left(\nabla F\left(\tilde{x}, \xi_{i_{t}}\right)-\nabla F\left(x^{*}, \xi_{i_{t}}\right)\right)\right\|_{2}^{2} \mid x_{t-1}\right) .
\end{aligned}
$$

Agora, por propriedade da variância, para qualquer variável aleatória $y$ se tem que $\mathbb{E}\left(\|y-\mathbb{E}(y)\|_{2}^{2}\right)=$ $\mathbb{E}\left(\|y\|_{2}^{2}\right)-\|\mathbb{E}(y)\|_{2}^{2} \leq \mathbb{E}\left(\|y\|_{2}^{2}\right)$. Substituindo em (2.25) 


$$
\begin{aligned}
\mathbb{E}\left(\left\|v_{t}\right\|_{2}^{2} \mid x_{t-1}\right) \leq & 2 \mathbb{E}\left(\left\|\nabla F\left(x_{t-1}, \xi_{i_{t}}\right)-\nabla F\left(x^{*}, \xi_{i_{t}}\right)\right\|_{2}^{2} \mid x_{t-1}\right)+ \\
& +2 \mathbb{E}\left(\left\|\nabla F\left(\tilde{x}, \xi_{i_{t}}\right)-\nabla F\left(x^{*}, \xi_{i_{t}}\right)\right\|_{2}^{2} \mid x_{t-1}\right) .
\end{aligned}
$$

Logo, usando a Proposição 2.3 .3 e o fato de $\nabla f\left(x^{*}\right)=0$ temos

$$
\mathbb{E}\left(\left\|v_{t}\right\|_{2}^{2} \mid x_{t-1}\right) \leq 4 L\left(f\left(x_{t-1}\right)-f\left(x^{*}\right)+f(\tilde{x})-f\left(x^{2}\right)\right) .
$$

Agora,

$$
\begin{aligned}
\mathbb{E}\left(v_{t} \mid x_{t-1}\right) & =\mathbb{E}\left(\nabla F\left(x_{t-1}, \xi_{i_{t}}\right)-\nabla F\left(\tilde{x}, \xi_{i_{t}}\right)+\tilde{\mu} \mid x_{t-1}\right) \\
& =\nabla f\left(x_{t-1}\right)-\nabla f(\tilde{x})+\tilde{\mu}=\nabla f\left(x_{t-1}\right) .
\end{aligned}
$$

Calculando $\mathbb{E}\left(\left\|x_{t}-x^{*}\right\|_{2}^{2} \mid x_{t-1}\right)$ obtemos

$$
\begin{aligned}
\mathbb{E}\left(\left\|x_{t}-x^{*}\right\|_{2}^{2} \mid x_{t-1}\right) & =\mathbb{E}\left(\left\|x_{t-1}-x^{*}-\eta\left(\nabla F\left(x_{t-1}, \xi_{i_{t}}\right)-\nabla F\left(\tilde{x}, \xi_{i_{t}}\right)+\tilde{\mu}\right)\right\|_{2}^{2} \mid x_{t-1}\right) \\
& =\mathbb{E}\left(\left\|x_{t-1}-x^{*}\right\|_{2}^{2}-2 \eta\left(x_{t-1}-x^{*}\right)^{T} v_{t}+\eta^{2}\left\|v_{t}\right\|_{2}^{2} \mid x_{t-1}\right) \\
& =\left\|x_{t-1}-x^{*}\right\|_{2}^{2}-2 \eta\left(x_{t-1}-x^{*}\right)^{T} \mathbb{E}\left(v_{t} \mid x_{t-1}\right)+\eta^{2} \mathbb{E}\left(\left\|v_{t}\right\|_{2}^{2} \mid x_{t-1}\right) .
\end{aligned}
$$

Considerando (2.28) e (2.27)

$$
\begin{aligned}
\mathbb{E}\left(\left\|x_{t}-x^{*}\right\|_{2}^{2} \mid x_{t-1}\right) \leq & \left\|x_{t-1}-x^{*}\right\|_{2}^{2}-2 \eta\left(x_{t-1}-x^{*}\right)^{T} \nabla f\left(x_{t-1}\right)+ \\
& +4 L \eta^{2}\left(f\left(x_{t-1}\right)-f\left(x^{*}\right)+f(\tilde{x})-f\left(x^{*}\right)\right) .
\end{aligned}
$$

Por convexidade de $f(x)$ se cumpre que $-\left(x_{t-1}-x^{*}\right)^{T} \nabla f\left(x_{t-1}\right) \leq f\left(x^{*}\right)-f\left(x_{t-1}\right)$. Assim,

$$
\begin{aligned}
\mathbb{E}\left(\left\|x_{t}-x^{*}\right\|_{2}^{2} \mid x_{t-1}\right) & \leq\left\|x_{t-1}-x^{*}\right\|_{2}^{2}+2 \eta\left(f\left(x^{*}\right)-f\left(x_{t-1}\right)\right)+4 L \eta^{2}\left(f\left(x_{t-1}\right)-f\left(x^{*}\right)+f(\tilde{x})-f\left(x^{*}\right)\right) \\
& =\left\|x_{t-1}-x^{*}\right\|_{2}^{2}-2 \eta(1-2 L \eta)\left(f\left(x_{t-1}\right)-f\left(x^{*}\right)\right)+4 L \eta^{2}\left(f(\tilde{x})-f\left(x^{*}\right)\right) .
\end{aligned}
$$

Consideremos uma iteração $s$ fixa tal que $\tilde{x}=\tilde{x}_{s-1}$ e $\tilde{x}_{s}$ é selecionado depois de que todas as $m$ atualizações aconteçam. Somando (2.31) por $t=1, \ldots, m$ e reescrevendo temos:

$\sum_{t=1}^{m} \mathbb{E}\left(\left\|x_{t}-x^{*}\right\|_{2}^{2} \mid x_{t-1}\right)+2 \eta(1-2 L \eta) \sum_{t=1}^{m}\left(f\left(x_{t-1}\right)-f\left(x^{*}\right)\right) \leq \sum_{t=1}^{m}\left\{\left\|x_{t-1}-x^{*}\right\|_{2}^{2}+4 L \eta^{2}\left(f(\tilde{x})-f\left(x^{*}\right)\right)\right\}$.

Por propriedade de valor esperado sabemos que $\mathbb{E}(\mathbb{E}(X \mid Y))=\mathbb{E}(X)$. Assim, tomando valor esperado em (2.32) obtemos:

$$
\begin{aligned}
\sum_{t=1}^{m} \mathbb{E}\left(\mathbb{E}\left(\left\|x_{t}-x^{*}\right\|_{2}^{2} \mid x_{t-1}\right)\right)+2 \eta(1-2 L \eta) \sum_{t=1}^{m} \mathbb{E}\left(f\left(x_{t-1}\right)-f\left(x^{*}\right)\right) & \leq \sum_{t=1}^{m} \mathbb{E}\left(\left\|x_{t-1}-x^{*}\right\|_{2}^{2}\right) \\
& +4 L \eta^{2} \sum_{t=1}^{m} \mathbb{E}\left(f(\tilde{x})-f\left(x^{*}\right)\right)
\end{aligned}
$$




$$
\sum_{t=1}^{m} \mathbb{E}\left(\left\|x_{t}-x^{*}\right\|_{2}^{2}\right)+2 \eta(1-2 L \eta) \sum_{t=1}^{m} \mathbb{E}\left(f\left(x_{t-1}\right)-f\left(x^{*}\right)\right) \leq \sum_{t=1}^{m} \mathbb{E}\left(\left\|x_{t-1}-x^{*}\right\|_{2}^{2}\right)+4 L \eta^{2} m \mathbb{E}\left(f(\tilde{x})-f\left(x^{*}\right)\right) .
$$

Usando a opção II da atualização e juntando com a Proposição 2.3.2,

$$
\begin{aligned}
\mathbb{E}\left(\left\|x_{m}-x^{*}\right\|_{2}^{2}\right)+2 \eta(1-2 L \eta) \sum_{t=1}^{m} \mathbb{E}\left(f\left(x_{t-1}\right)-f\left(x^{*}\right)\right) & \leq \mathbb{E}\left(\left\|x_{0}-x^{*}\right\|_{2}^{2}\right)+4 L \eta^{2} m \mathbb{E}\left(f(\tilde{x})-f\left(x^{*}\right)\right) \\
\mathbb{E}\left(\left\|x_{m}-x^{*}\right\|_{2}^{2}\right)+2 \eta(1-2 L \eta) m \mathbb{E}\left(f\left(\tilde{x}_{s}\right)-f\left(x^{*}\right)\right) & \leq \mathbb{E}\left(\left\|x_{0}-x^{*}\right\|_{2}^{2}\right)+4 L \eta^{2} m \mathbb{E}\left(f(\tilde{x})-f\left(x^{*}\right)\right) \\
& \leq \frac{2}{\gamma} \mathbb{E}\left(f(\tilde{x})-f\left(x^{*}\right)\right)+4 L \eta^{2} m \mathbb{E}\left(f(\tilde{x})-f\left(x^{*}\right)\right) \\
& =2\left(\gamma^{-1}+2 L \eta^{2} m\right) \mathbb{E}\left(f(\tilde{x})-f\left(x^{*}\right)\right) .
\end{aligned}
$$

Assim, considerando $\tilde{x}=\tilde{x}_{s-1}$ obtemos

$$
\mathbb{E}\left(f\left(\tilde{x}_{s}\right)-f\left(x^{*}\right)\right) \leq\left[\frac{1}{\gamma \eta(1-2 L \eta) m}+\frac{2 L \eta}{1-2 L \eta}\right] \mathbb{E}\left(f\left(\tilde{x}_{s-1}\right)-f\left(x^{*}\right)\right) .
$$

Que implica, finalmente

$$
\mathbb{E}\left(f\left(\tilde{x}_{s}\right)-f\left(x^{*}\right)\right) \leq \alpha^{s} \mathbb{E}\left(f\left(\tilde{x}_{s-1}\right)-f\left(x^{*}\right)\right) .
$$

\subsubsection{SAGA}

Consideremos a seguinte modificação do problema (2.12) para o caso geral:

$$
\min _{x \in \mathbb{R}^{d}} \frac{1}{N} \sum_{i=1}^{N} F\left(x, \xi_{i}\right)+h(x)
$$

onde $h: \mathbb{R}^{d} \rightarrow \mathbb{R}$ é uma função de regularização convexa. O Algoritmo 2.3.2 [DBLJ14] a apresentar, segue o mesmo análise estudado em Stochastic Variance Reduced Gradient, diferenciando-se unicamente na escolha de $W$.

Especificamente, seja $\phi$ uma lista que guarda para cada $j \in\{1, \ldots, N\}$ o avance de $x$ cada vez que $j$ é selecionado como índice para a estimação do gradiente estocástico $\nabla F\left(x, \xi_{j}\right)$ de $\nabla \hat{f}_{N}(x)$, isto é, cada $\phi_{j}^{k}$ refere-se ao último valor obtido até a iteração $k$ na minimização de $\hat{f}_{N}(x)$ usando o índice $j$ na estimação do gradiente. Assim, se define $W=\nabla F\left(\phi_{j}^{k}, \xi_{j}\right)$ e a Control Variate $Z=Y-W+\mathbb{E}(W)$ é um estimador consistente de $Y=\nabla F\left(x, \xi_{j}\right)$ com variância menor. 
Algoritmo 2.3.2. SAGA

Entrada: $x_{0} \in \mathbb{R}^{d}$; $\gamma$ : learning rate.

Passo 1. Inicialize $\phi_{i}^{0}=x_{0} \forall i \in\{1, \ldots, N\}$

Passo 2. $k=0$

Passo 3. Escolha aleatoriamente $j \in\{1, \ldots, N\}$

Passo 4. $\phi_{j}^{k+1}=x_{k}$

Passo 5. $w_{k+1}=x_{k}-\gamma\left(\nabla F\left(\phi_{j}^{k+1}, \xi_{j}\right)-\nabla F\left(\phi_{j}^{k}, \xi_{j}\right)+\frac{1}{N} \sum_{i=1}^{N} \nabla F\left(\phi_{i}^{k}, \xi_{i}\right)\right)$

Passo 6. $x_{k+1}=\operatorname{prox}_{\gamma}^{h}\left(w_{k+1}\right)$

Passo 7. $k \leftarrow k+1$

Passo 8. Volte ao Passo 3 até satisfazer condição de parada.

onde $\operatorname{prox}_{\gamma}^{h}(y)=\underset{x \in \mathbb{R}^{d}}{\operatorname{argmin}}\left\{h(x)+\frac{1}{2 \gamma}\|x-y\|^{2}\right\}$. Para o caso que se tem estudado ao longo deste trabalho, i.e. $h(x)=0$, o Passo 6 é eliminado, e o Passo 5 é modificado a

$$
x_{k+1}=x_{k}-\gamma\left(\nabla F\left(\phi_{j}^{k+1}, \xi_{j}\right)-\nabla F\left(\phi_{j}^{k}, \xi_{j}\right)+\frac{1}{N} \sum_{i=1}^{N} \nabla F\left(\phi_{i}^{k}, \xi_{i}\right)\right) .
$$

As seguintes proposições enunciam propriedades de funções convexas, as quais são utilizadas no Teorema 2.3.2 o qual auxilia na demonstração de convergência do Algoritmo 2.3.2 descrita no Corolario 2.3.1.

Proposição 2.3.4. [DBLJ14] Seja $f(x)=\frac{1}{n} \sum_{i=1}^{n} F\left(x, \xi_{i}\right)$. Suponha que cada $F\left(x, \xi_{i}\right)$ é fortemente convexa com parâmetro $\mu$ e possue gradiente Lipschitz com constante L. Então, para todo $x, x^{*}$ temos:

$$
\begin{aligned}
\nabla f(x)^{T}\left(x^{*}-x\right) \leq & \frac{L-\mu}{L}\left(f\left(x^{*}\right)-f(x)\right)-\frac{\mu}{2}\left\|x^{*}-x\right\|^{2}+ \\
& \quad-\frac{1}{2 L n} \sum_{i=1}^{n}\left\|\nabla F\left(x^{*}, \xi_{i}\right)-\nabla F\left(x, \xi_{i}\right)\right\|^{2}-\frac{\mu}{L} \nabla f\left(x^{*}\right)^{T}\left(x-x^{*}\right) .
\end{aligned}
$$

Proposição 2.3.5. [DBLJ14] Para todo $\phi_{i}, x^{*}$ se satisfaz que

$$
\frac{1}{n} \sum_{i=1}^{n}\left\|\nabla F\left(\phi_{i}, \xi_{i}\right)-\nabla F\left(x^{*}, \xi_{i}\right)\right\|^{2} \leq 2 L\left[\frac{1}{n} \sum_{i=1}^{n} F\left(\phi_{i}, \xi_{i}\right)-f\left(x^{*}\right)-\frac{1}{n} \sum_{i=1}^{n} \nabla F\left(x^{*}, \xi_{i}\right)^{T}\left(\phi_{i}-x^{*}\right)\right] .
$$

Proposição 2.3.6. [DBLJ14] Para todo $\phi_{i}^{k}, x^{*}, x_{k}$ e $\beta>0$, com $w_{k+1}$ definido como no Passo 5, se satisfaz que (condicional em $x_{k}, \phi_{i}^{k}$ )

$$
\begin{aligned}
\mathbb{E}\left(\left\|w_{k+1}-x_{k}-\gamma \nabla f\left(x^{*}\right)\right\|^{2}\right) \leq & \gamma^{2}\left(1+\beta^{-1}\right) \mathbb{E}\left(\left\|\nabla F\left(\phi_{j}^{k}, \xi_{j}\right)-\nabla F\left(x^{*}, \xi_{j}\right)\right\|^{2}\right) \\
& +\gamma^{2}(1+\beta) \mathbb{E}\left(\left\|\nabla F\left(x_{k}, \xi_{j}\right)-\nabla F\left(x^{*}, \xi_{j}\right)\right\|^{2}\right)-\gamma^{2} \beta\left\|\nabla f\left(x_{k}\right)-\nabla f\left(x^{*}\right)\right\|^{2} .
\end{aligned}
$$

Demonstração. (Todos os valores esperados nesta demonstração são condicionais nos valores de 
$\left.x_{k}, \phi_{i}^{k}\right)$

$\mathbb{E}\left(\left\|w_{k+1}-x_{k}+\gamma \nabla f\left(x^{*}\right)\right\|^{2}\right)=\mathbb{E}\left(\left\|x_{k}-\gamma\left[\nabla F\left(x_{k}, \xi_{j}\right)-\nabla F\left(\phi_{j}^{k}, \xi_{j}\right)+\frac{1}{n} \sum_{i=1}^{n} F\left(\phi_{i}^{k}, \xi_{i}\right)\right]-x_{k}+\gamma \nabla f\left(x^{*}\right)\right\|^{2}\right)$ $=\gamma^{2} \mathbb{E}\left(\left\|-\nabla F\left(x_{k}, \xi_{j}\right)+\nabla F\left(\phi_{j}^{k}, \xi_{j}\right)-\frac{1}{n} \sum_{i=1}^{n} F\left(\phi_{i}^{k}, \xi_{i}\right)+\nabla f\left(x^{*}\right)\right\|^{2}\right)$.

Definamos $Y=-\nabla F\left(x_{k}, \xi_{j}\right)+\nabla F\left(\phi_{j}^{k}, \xi_{j}\right)-\frac{1}{n} \sum_{i=1}^{n} F\left(\phi_{i}^{k}, \xi_{i}\right)+\nabla f\left(x^{*}\right)$. Temos que,

$$
\begin{aligned}
\mathbb{E}(Y) & =-\frac{1}{n} \sum_{i=1}^{n} \nabla F\left(x_{k}, \xi_{j}\right)+\frac{1}{n} \sum_{i=1}^{n} \nabla F\left(\phi_{i}^{k}, \xi_{i}\right)-\frac{1}{n} \sum_{i=1}^{n} F\left(\phi_{i}^{k}, \xi_{i}\right)+\nabla f\left(x^{*}\right) \\
& =\nabla f\left(x^{*}\right)-\frac{1}{n} \sum_{i=1}^{n} F\left(x_{k}, \xi_{j}\right) .
\end{aligned}
$$

Logo, por propiedade de variância, se satisfaz que $\mathbb{E}\left(\|Y\|^{2}\right)=\mathbb{E}\left(\|Y-\mathbb{E}(Y)\|^{2}\right)+(\mathbb{E}(Y))^{2}$, assim, voltando em 2.35

$$
\begin{aligned}
\mathbb{E}\left(\left\|w_{k+1}-x_{k}+\gamma \nabla f\left(x^{*}\right)\right\|^{2}\right)= & \gamma^{2} \mathbb{E}\left(\left\|-\nabla F\left(x_{k}, \xi_{j}\right)+\nabla F\left(\phi_{j}^{k}, \xi_{j}\right)-\frac{1}{n} \sum_{i=1}^{n} F\left(\phi_{i}^{k}, \xi_{i}\right)+\frac{1}{n} \sum_{i=1}^{n} F\left(x_{k}, \xi_{j}\right)\right\|^{2}\right) \\
& +\gamma^{2}\left\|\nabla f\left(x^{*}\right)-\frac{1}{n} \sum_{i=1}^{n} F\left(x_{k}, \xi_{j}\right)\right\|^{2}
\end{aligned}
$$

Sabemos que $\forall \beta>0$ se satisfaz que $\|a+b\|^{2} \leq\left(1+\beta^{-1}\right)\|a\|^{2}+(1+\beta)\|b\|^{2}$. Assim, considerando

$$
\begin{aligned}
& a=\nabla F\left(\phi_{j}^{k}, \xi_{j}\right)-\nabla F\left(x^{*}, \xi_{j}\right)-\frac{1}{n} \sum_{i=1}^{n} F\left(\phi_{i}^{k}, \xi_{i}\right)+\nabla f\left(x^{*}\right) \\
& b=-\nabla F\left(x_{k}, \xi_{j}\right)+\nabla F\left(x^{*}, \xi_{j}\right)+\nabla f\left(x_{k}\right)-\nabla f\left(x^{*}\right)
\end{aligned}
$$

e substituindo em 2.36 temos,

$$
\begin{gathered}
\mathbb{E}\left(\left\|w_{k+1}-x_{k}+\gamma \nabla f\left(x^{*}\right)\right\|^{2}\right) \leq \\
\gamma^{2}\left(1+\beta^{-1}\right) \mathbb{E}\left(\left\|\nabla F\left(\phi_{j}^{k}, \xi_{j}\right)-\nabla F\left(x^{*}, \xi_{j}\right)-\left(\frac{1}{n} \sum_{i=1}^{n} F\left(\phi_{i}^{k}, \xi_{i}\right)-\nabla f\left(x^{*}\right)\right)\right\|^{2}\right) \\
+\gamma^{2}(1+\beta) \mathbb{E}\left(\left\|-\left\{\nabla F\left(x_{k}, \xi_{j}\right)-\nabla F\left(x^{*}, \xi_{j}\right)-\left(\nabla f\left(x_{k}\right)-\nabla f\left(x^{*}\right)\right)\right\}\right\|^{2}\right) \\
+\gamma^{2}\left\|\nabla f\left(x^{*}\right)-\frac{1}{n} \sum_{i=1}^{n} F\left(x_{k}, \xi_{j}\right)\right\|^{2} .
\end{gathered}
$$

Agora, pode-se observar que os dois valores esperados em 2.37 estão na forma $\mathbb{E}\left(\|Z-\mathbb{E}(Z)\|^{2}\right)$, assim, usando novamente a propriedade de variância, 
- $\mathbb{E}\left(\left\|\nabla F\left(\phi_{j}^{k}, \xi_{j}\right)-\nabla F\left(x^{*}, \xi_{j}\right)-\left(\frac{1}{n} \sum_{i=1}^{n} F\left(\phi_{i}^{k}, \xi_{i}\right)-\nabla f\left(x^{*}\right)\right)\right\|^{2}\right)=\mathbb{E}\left(\left\|\nabla F\left(\phi_{j}^{k}, \xi_{j}\right)-\nabla F\left(x^{*}, \xi_{j}\right)\right\|^{2}\right)$

$$
-\left\|\frac{1}{n} \sum_{i=1}^{n} F\left(\phi_{i}^{k}, \xi_{i}\right)-\nabla f\left(x^{*}\right)\right\|^{2} \text {. }
$$

- $\mathbb{E}\left(\left\|\nabla F\left(x_{k}, \xi_{j}\right)-\nabla F\left(x^{*}, \xi_{j}\right)-\left(\nabla f\left(x_{k}\right)-\nabla f\left(x^{*}\right)\right)\right\|^{2}\right)=\mathbb{E}\left(\left\|\nabla F\left(x_{k}, \xi_{j}\right)-\nabla F\left(x^{*}, \xi_{j}\right)\right\|^{2}\right)$

$$
-\left\|\nabla f\left(x_{k}\right)-\nabla f\left(x^{*}\right)\right\|^{2} \text {. }
$$

Finalmente, voltando em 2.37

$$
\begin{aligned}
\mathbb{E}\left(\left\|w_{k+1}-x_{k}+\gamma \nabla f\left(x^{*}\right)\right\|^{2}\right) \leq & \gamma^{2}\left(1+\beta^{-1}\right) \mathbb{E}\left(\left\|\nabla F\left(\phi_{j}^{k}, \xi_{j}\right)-\nabla F\left(x^{*}, \xi_{j}\right)\right\|^{2}\right) \\
& -\gamma^{2}\left(1+\beta^{-1}\right)\left\|\frac{1}{n} \sum_{i=1}^{n} F\left(\phi_{i}^{k}, \xi_{i}\right)-\nabla f\left(x^{*}\right)\right\|^{2} \\
& +\gamma^{2}(1+\beta) \mathbb{E}\left(\left\|\nabla F\left(x_{k}, \xi_{j}\right)-\nabla F\left(x^{*}, \xi_{j}\right)\right\|^{2}\right) \\
& -\gamma^{2}(1+\beta)\left\|\nabla f\left(x_{k}\right)-\nabla f\left(x^{*}\right)\right\|^{2} \\
& +\gamma^{2}\left\|\nabla f\left(x^{*}\right)-\frac{1}{n} \sum_{i=1}^{n} F\left(x_{k}, \xi_{j}\right)\right\|^{2} \\
= & \gamma^{2}\left(1+\beta^{-1}\right) \mathbb{E}\left(\left\|\nabla F\left(\phi_{j}^{k}, \xi_{j}\right)-\nabla F\left(x^{*}, \xi_{j}\right)\right\|^{2}\right) \\
& -\gamma^{2}\left(1+\beta^{-1}\right)\left\|\frac{1}{n} \sum_{i=1}^{n} F\left(\phi_{i}^{k}, \xi_{i}\right)-\nabla f\left(x^{*}\right)\right\|^{2} \\
& +\gamma^{2}(1+\beta) \mathbb{E}\left(\left\|\nabla F\left(x_{k}, \xi_{j}\right)-\nabla F\left(x^{*}, \xi_{j}\right)\right\|^{2}\right) \\
& -\gamma^{2} \beta\left\|\nabla f\left(x_{k}\right)-\nabla f\left(x^{*}\right)\right\|^{2} \\
\leq & \gamma^{2}\left(1+\beta^{-1}\right) \mathbb{E}\left(\left\|\nabla F\left(\phi_{j}^{k}, \xi_{j}\right)-\nabla F\left(x^{*}, \xi_{j}\right)\right\|^{2}\right) \\
& +\gamma^{2}(1+\beta) \mathbb{E}\left(\left\|\nabla F\left(x_{k}, \xi_{j}\right)-\nabla F\left(x^{*}, \xi_{j}\right)\right\|^{2}\right) \\
& -\gamma^{2} \beta\left\|\nabla f\left(x_{k}\right)-\nabla f\left(x^{*}\right)\right\|^{2} .
\end{aligned}
$$

Teorema 2.3.2. [DBLJ14] Defina a função Lyapunov T como

$$
T^{k}:=T\left(x_{k},\left\{\phi_{i}^{k}\right\}_{i=1}^{n}\right):=\frac{1}{n} \sum_{i} F\left(\phi_{i}^{k}, \xi_{i}\right)-f\left(x^{*}\right)-\frac{1}{n} \sum_{i} \nabla F\left(x^{*}, \xi_{i}\right)^{T}\left(\phi_{i}^{k}-x^{*}\right)+c\left\|x_{k}-x^{*}\right\|^{2} .
$$

Então, com $\gamma=\frac{1}{2(\mu n+L)}, c=\frac{1}{2 \gamma(1-\gamma \mu) n}$ e $\kappa=\frac{1}{\gamma \mu}$, temos o seguinte cambio esperado da função Lyapunov entre passos do Algoritmo 2.3.2 (condicional em $T^{k}$ ):

$$
\mathbb{E}\left(T^{k+1}\right) \leq\left(1-\frac{1}{\kappa}\right) T^{k}
$$

Demonstração. Calculando o $\mathbb{E}\left(T^{k+1} \mid T^{k}\right)$ por partes: 
- $\mathbb{E}\left(\frac{1}{n} \sum_{i} F\left(\phi_{i}^{k+1}, \xi_{i}\right) \mid T^{k}\right)=\frac{1}{n} \mathbb{E}\left(\sum_{i \neq j} F\left(\phi_{i}^{k}, \xi_{i}\right)+F\left(x_{k}, \xi_{j}\right) \mid T^{k}\right)$

$$
\begin{aligned}
& =\frac{1}{n}\left((n-1) \sum_{i} F\left(\phi_{i}^{k}, \xi_{i}\right)+f\left(x_{k}\right)\right) \\
& =\frac{1}{n} f\left(x_{k}\right)+\left(1-\frac{1}{n}\right) \sum_{i} F\left(\phi_{i}^{k}, \xi_{i}\right) .
\end{aligned}
$$

- $\mathbb{E}\left(-\frac{1}{n} \sum_{i} \nabla F\left(x^{*}, \xi_{i}\right)^{T}\left(\phi_{i}^{k+1}-x^{*}\right) \mid T^{k}\right)=-\frac{1}{n} \mathbb{E}\left(\sum_{i \neq j} \nabla F\left(x^{*}, \xi_{i}\right)^{T}\left(\phi_{i}^{k}-x^{*}\right) \mid T^{k}\right)$

$$
\begin{aligned}
& -\mathbb{E}\left(\frac{1}{n} \nabla F\left(x^{*}, \xi_{j}\right)^{T}\left(x_{k}-x^{*}\right) \mid T^{k}\right) \\
=- & \frac{1}{n} \sum_{i \neq j} \mathbb{E}\left(\nabla F\left(x^{*}, \xi_{i}\right) \mid T^{k}\right)^{T}\left(\phi_{i}^{k}-x^{*}\right) \\
& -\frac{1}{n} \mathbb{E}\left(\nabla F\left(x^{*}, \xi_{j}\right) \mid T^{k}\right)^{T}\left(x_{k}-x^{*}\right) \\
=- & \frac{1}{n} \sum_{i \neq j} \frac{1}{n} \sum_{i=1}^{n}\left(\nabla F\left(x^{*}, \xi_{i}\right)\right)^{T}\left(\phi_{i}^{k}-x^{*}\right)+ \\
& -\frac{1}{n} \nabla f\left(x^{*}\right)^{T}\left(x_{k}-x^{*}\right) \\
=- & \left(1-\frac{1}{n}\right)^{\frac{1}{n}} \sum_{i=1}^{n}\left(\nabla F\left(x^{*}, \xi_{i}\right)\right)^{T}\left(\phi_{i}^{k}-x^{*}\right)+ \\
& -\frac{1}{n} \nabla f\left(x^{*}\right)^{T}\left(x_{k}-x^{*}\right) .
\end{aligned}
$$

- $\mathbb{E}\left(c\left\|x_{k+1}-x^{*}\right\|^{2} \mid T^{k}\right)$ : Por propriedades do operador proximal, temos que, se $x^{*}$ é minimizador, então $x^{*}=\operatorname{prox}_{\gamma}\left(x^{*}\right)$. Logo, como $\nabla f\left(x^{*}\right)=0$, então podemos reescrever a igualdade como $x^{*}=\operatorname{prox}_{\gamma}\left(x^{*}-\gamma \nabla f\left(x^{*}\right)\right)$. Assim:

$$
\begin{aligned}
c\left\|x_{k+1}-x^{*}\right\|^{2} & =c\left\|\operatorname{prox}_{\gamma}\left(w_{k+1}\right)-\operatorname{prox}_{\gamma}\left(x^{*}-\gamma \nabla f\left(x^{*}\right)\right)\right\|^{2} \\
& \leq c\left\|w_{k+1}-x^{*}+\gamma \nabla f\left(x^{*}\right)\right\|^{2} .
\end{aligned}
$$

Sabemos que

$$
\begin{aligned}
\mathbb{E}\left(w_{k+1} \mid T^{k}\right) & =\mathbb{E}\left(x_{k}-\gamma\left[\nabla F\left(x_{k}, \xi_{j}\right)-\nabla F\left(\phi_{j}^{k}, \xi_{j}\right)+\frac{1}{n} \sum_{i=1}^{n} \nabla f\left(\phi_{i}^{k}, \xi_{i}\right)\right] \mid T^{k}\right) \\
& =x_{k}-\gamma\left[\nabla f\left(x_{k}\right)-\frac{1}{n} \sum_{i=1}^{n} \nabla f\left(\phi_{i}^{k}, \xi_{i}\right)+\frac{1}{n} \sum_{i=1}^{n} \nabla f\left(\phi_{i}^{k}, \xi_{i}\right)\right] \\
& =x_{k}-\gamma \nabla f\left(x_{k}\right) .
\end{aligned}
$$


Assim, tomando o valor esperado em (2.38) obtemos

$$
\begin{aligned}
\mathbb{E}\left(c\left\|x_{k+1}-x^{*}\right\|^{2} \mid T^{k}\right) \leq & \mathbb{E}\left(c\left\|w_{k+1}-x^{*}+\gamma \nabla f\left(x^{*}\right)\right\|^{2} \mid T^{k}\right) \\
= & c \mathbb{E}\left(\left\|x_{k}-x^{*}+w_{k+1}-x_{k}+\gamma \nabla f\left(x^{*}\right)\right\|^{2} \mid T^{k}\right) \\
= & c \mathbb{E}\left(\left\|x_{k}-x^{*}\right\|^{2} \mid T^{k}\right)+ \\
& \quad+c \mathbb{E}\left(2\left(x_{k}-x^{*}\right)^{T}\left(w_{k+1}-x_{k}+\gamma \nabla f\left(x^{*}\right)\right) \mid T^{k}\right)+ \\
& \quad+c \mathbb{E}\left(\left\|w_{k+1}-x_{k}+\gamma \nabla f\left(x^{*}\right)\right\|^{2} \mid T^{k}\right) \\
= & c\left\|x_{k}-x^{*}\right\|^{2}+ \\
& +2 c\left(x_{k}-x^{*}\right)^{T}\left(\mathbb{E}\left(w_{k+1} \mid T^{k}\right)-x_{k}+\gamma \nabla f\left(x^{*}\right)\right)+ \\
& +c \mathbb{E}\left(\left\|w_{k+1}-x_{k}+\gamma \nabla f\left(x^{*}\right)\right\|^{2} \mid T^{k}\right) .
\end{aligned}
$$

Substituindo (2.39) e a Proposição 2.3.6 obtemos que

$$
\begin{aligned}
\mathbb{E}\left(c\left\|x_{k+1}-x^{*}\right\|^{2} \mid T^{k}\right) \leq & \left\|x_{k}-x^{*}\right\|^{2}+ \\
& +2 c\left(x_{k}-x^{*}\right)^{T}\left(x_{k}-\gamma \nabla f\left(x_{k}\right)-x_{k}+\gamma \nabla f\left(x^{*}\right)\right)+ \\
& +c \gamma^{2}\left(1+\beta^{-1}\right) \mathbb{E}\left(\left\|\nabla F\left(\phi_{j}^{k}, \xi_{j}\right)-\nabla F\left(x^{*}, \xi_{j}\right)\right\|^{2} \mid T^{k}\right) \\
& +c \gamma^{2}(1+\beta) \mathbb{E}\left(\left\|\nabla F\left(x_{k}, \xi_{j}\right)-\nabla F\left(x^{*}, \xi_{j}\right)\right\|^{2} \mid T^{k}\right) \\
& -c \gamma^{2} \beta\left\|\nabla f\left(x_{k}\right)-\nabla f\left(x^{*}\right)\right\|^{2} \\
\leq & \left\|x_{k}-x^{*}\right\|^{2}+ \\
& -2 c \gamma\left(x_{k}-x^{*}\right)^{T}\left(\nabla f\left(x_{k}\right)-\nabla f\left(x^{*}\right)\right)+ \\
& +c \gamma^{2}\left(1+\beta^{-1}\right) \mathbb{E}\left(\left\|\nabla F\left(\phi_{j}^{k}, \xi_{j}\right)-\nabla F\left(x^{*}, \xi_{j}\right)\right\|^{2} \mid T^{k}\right) \\
& +c \gamma^{2}(1+\beta) \mathbb{E}\left(\left\|\nabla F\left(x_{k}, \xi_{j}\right)-\nabla F\left(x^{*}, \xi_{j}\right)\right\|^{2} \mid T^{k}\right) \\
& -c \gamma^{2} \beta\left\|\nabla f\left(x_{k}\right)-\nabla f\left(x^{*}\right)\right\|^{2} .
\end{aligned}
$$

Substituindo a Proposição 2.3.4 e $\mathbb{E}\left(\left\|\nabla F\left(\phi_{i}^{k}, \xi_{j}\right)-\nabla F\left(x^{*}, \xi_{j}\right)\right\|^{2} \mid T^{k}\right)=\frac{1}{n} \sum_{i=1}^{n} \| \nabla F\left(\phi_{i}^{k}, \xi_{i}\right)-$ $\nabla F\left(x^{*}, \xi_{i}\right) \|^{2}$ para usar a Proposição 2.3 .5 em (2.41) obtemos: 


$$
\begin{aligned}
\mathbb{E}\left(c\left\|x_{k+1}-x^{*}\right\|^{2} \mid T^{k}\right) \leq & \left\|x_{k}-x^{*}\right\|^{2}+2 c \gamma \frac{L-\mu}{L}\left(f\left(x^{*}\right)-f\left(x_{k}\right)\right)-2 c \gamma \frac{\mu}{2}\left\|x^{*}-x_{k}\right\|^{2}+ \\
& -2 c \gamma \frac{1}{2 L n} \sum_{i=1}^{n}\left\|\nabla F\left(x^{*}, \xi_{i}\right)-\nabla F\left(x_{k}, \xi_{i}\right)\right\|^{2} \\
& -2 c \gamma \frac{\mu}{L} \nabla f\left(x^{*}\right)^{T}\left(x_{k}-x^{*}\right)-2 c \gamma \nabla f\left(x^{*}\right)^{T}\left(x_{k}-x^{*}\right) \\
& +c \gamma^{2}\left(1+\beta^{-1}\right) 2 L \frac{1}{n} \sum_{i=1}^{n} F\left(\phi_{i}, \xi_{i}\right)-f\left(x^{*}\right) \\
& -c \gamma^{2}\left(1+\beta^{-1}\right) 2 L \frac{1}{n} \sum_{i=1}^{n} \nabla F\left(x^{*}, \xi_{i}\right)^{T}\left(\phi_{i}-x^{*}\right) \\
& +c \gamma^{2}(1+\beta) \mathbb{E}\left(\left\|\nabla F\left(x_{k}, \xi_{j}\right)-\nabla F\left(x^{*}, \xi_{j}\right)\right\|^{2} \mid T^{k}\right) \\
& -c \gamma^{2} \beta\left\|\nabla f\left(x_{k}\right)-\nabla f\left(x^{*}\right)\right\|^{2} \\
\leq & (c-c \gamma \mu)\left\|x_{k}-x^{*}\right\|^{2}+ \\
& -2 c \gamma \frac{L-\mu}{L}\left(f\left(x_{k}\right)-f\left(x^{*}\right)-\nabla f\left(x^{*}\right)^{T}\left(x_{k}-x^{*}\right)\right) \\
& \left(c \gamma^{2}(1+\beta)-c \gamma \frac{1}{L}\right) \mathbb{E}\left(\left\|\nabla F\left(x_{k}, \xi_{j}\right)-\nabla F\left(x^{*}, \xi_{j}\right)\right\|^{2} \mid T^{k}\right) \\
& +2 c \gamma^{2}\left(1+\beta^{-1}\right) L \frac{1}{n} \sum_{i=1}^{n} F\left(\phi_{i}, \xi_{i}\right)-f\left(x^{*}\right) \\
& -2 c \gamma^{2}\left(1+\beta^{-1}\right) L \frac{1}{n} \sum_{i=1}^{n} \nabla F\left(x^{*}, \xi_{i}\right)^{T}\left(\phi_{i}-x^{*}\right) \\
& -c \gamma^{2} \beta\left\|\nabla f\left(x_{k}\right)-\nabla f\left(x^{*}\right)\right\|^{2} . \\
&
\end{aligned}
$$

Finalmente, juntando as três equações anteriores e substraindo $T^{k}$ em ambos lados temos que: 


$$
\begin{aligned}
& \mathbb{E}\left(T^{k+1} \mid T^{k}\right)-T^{k} \leq \frac{1}{n} f\left(x_{k}\right)+\left(1-\frac{1}{n}\right) \frac{1}{n} \sum_{i} F\left(\phi_{i}^{k}, \xi_{i}\right) \\
& -\left(1-\frac{1}{n}\right) \frac{1}{n} \sum_{i=1}^{n} \nabla F\left(x^{*}, \xi_{i}\right)^{T}\left(\phi_{i}^{k}-x^{*}\right)-\frac{1}{n} \nabla f\left(x^{*}\right)^{T}\left(x_{k}-x^{*}\right) \\
& +(c-c \gamma \mu)\left\|x_{k}-x^{*}\right\|^{2}+ \\
& -2 c \gamma \frac{L-\mu}{L}\left(f\left(x_{k}\right)-f\left(x^{*}\right)-\nabla f\left(x^{*}\right)^{T}\left(x_{k}-x^{*}\right)\right) \\
& +\left(c \gamma^{2}(1+\beta)-c \gamma \frac{1}{L}\right) \mathbb{E}\left(\left\|\nabla F\left(x_{k}, \xi_{j}\right)-\nabla F\left(x^{*}, \xi_{j}\right)\right\|^{2} \mid T^{k}\right) \\
& +2 c \gamma^{2}\left(1+\beta^{-1}\right) L \frac{1}{n} \sum_{i=1}^{n} F\left(\phi_{i}^{k}, \xi_{i}\right)-f\left(x^{*}\right) \\
& -2 c \gamma^{2}\left(1+\beta^{-1}\right) L \frac{1}{n} \sum_{i=1}^{n} \nabla F\left(x^{*}, \xi_{i}\right)^{T}\left(\phi_{i}^{k}-x^{*}\right) \\
& -c \gamma^{2} \beta\left\|\nabla f\left(x_{k}\right)-\nabla f\left(x^{*}\right)\right\|^{2} \\
& -\left(\frac{1}{n} \sum_{i} F\left(\phi_{i}^{k}, \xi_{i}\right)-f\left(x^{*}\right)-\frac{1}{n} \sum_{i} \nabla F\left(x^{*}, \xi_{i}\right)^{T}\left(\phi_{i}^{k}-x^{*}\right)+c\left\|x_{k}-x^{*}\right\|^{2}\right) \\
& =\left(\frac{1}{n}-2 c \gamma \frac{L-\mu}{L}\right)\left[f\left(x_{k}\right)-f\left(x^{*}\right)-\nabla f\left(x^{*}\right)^{T}\left(x_{k}-x^{*}\right)\right] \\
& +\left(2 c \gamma^{2}\left(1+\beta^{-1}\right) L-\frac{1}{n}\right)\left[\frac{1}{n} \sum_{i=1}^{n} F\left(\phi_{i}^{k}, \xi_{i}\right)-f\left(x^{*}\right)-\frac{1}{n} \sum_{i=1}^{n} \nabla F\left(x^{*}, \xi_{i}\right)^{T}\left(\phi_{i}^{k}-x^{*}\right)\right] \\
& -c \gamma \mu\left\|x_{k}-x^{*}\right\|^{2}-c \gamma^{2} \beta\left\|\nabla f\left(x_{k}\right)-\nabla f\left(x^{*}\right)\right\|^{2} \\
& c \gamma\left(\gamma(1+\beta)-\frac{1}{L}\right) \mathbb{E}\left(\left\|\nabla F\left(x_{k}, \xi_{j}\right)-\nabla F\left(x^{*}, \xi_{j}\right)\right\|^{2} \mid T^{k}\right) .
\end{aligned}
$$

A última igualdade resultou do fato de adicionar e substrair $\frac{1}{n} f\left(x^{*}\right)$. Logo, como $f(x)$ é fortemente convexa, se satifaz que

$$
-\left\|\nabla f\left(x_{k}\right)-\nabla f\left(x^{*}\right)\right\|^{2} \leq-2 \mu\left[f\left(x_{k}\right)-f\left(x^{*}\right)-\nabla f\left(x^{*}\right)^{T}\left(x_{k}-x^{*}\right)\right] .
$$

Substituindo em (2.43) e somando e substraindo $\frac{1}{\kappa} T^{k}$ temos: 


$$
\begin{aligned}
\mathbb{E}\left(T^{k+1} \mid T^{k}\right)-T^{k} & \leq-\frac{1}{\kappa} T^{k}+\left(\frac{1}{n}-2 c \gamma \frac{L-\mu}{L}\right)\left[f\left(x_{k}\right)-f\left(x^{*}\right)-\nabla f\left(x^{*}\right)^{T}\left(x_{k}-x^{*}\right)\right] \\
& +\left(\frac{1}{\kappa}+2 c \gamma^{2}\left(1+\beta^{-1}\right) L-\frac{1}{n}\right)\left[\frac{1}{n} \sum_{i=1}^{n} F\left(\phi_{i}^{k}, \xi_{i}\right)-f\left(x^{*}\right)-\frac{1}{n} \sum_{i=1}^{n} \nabla F\left(x^{*}, \xi_{i}\right)^{T}\left(\phi_{i}^{k}-x^{*}\right)\right] \\
& +\left(\frac{1}{\kappa}-\gamma \mu\right) c\left\|x_{k}-x^{*}\right\|^{2}-2 c \gamma^{2} \beta \mu\left[f\left(x_{k}\right)-f\left(x^{*}\right)-\nabla f\left(x^{*}\right)^{T}\left(x_{k}-x^{*}\right)\right] \\
& +c \gamma\left(\gamma(1+\beta)-\frac{1}{L}\right) \mathbb{E}\left(\left\|\nabla F\left(x_{k}, \xi_{j}\right)-\nabla F\left(x^{*}, \xi_{j}\right)\right\|^{2} \mid T^{k}\right) \\
= & -\frac{1}{\kappa} T^{k}+\left(\frac{1}{n}-2 c \gamma \frac{L-\mu}{L}-2 c \gamma^{2} \beta \mu\right)\left[f\left(x_{k}\right)-f\left(x^{*}\right)-\nabla f\left(x^{*}\right)^{T}\left(x_{k}-x^{*}\right)\right] \\
& +\left(\frac{1}{\kappa}+2 c \gamma^{2}\left(1+\beta^{-1}\right) L-\frac{1}{n}\right)\left[\frac{1}{n} \sum_{i=1}^{n} F\left(\phi_{i}^{k}, \xi_{i}\right)-f\left(x^{*}\right)-\frac{1}{n} \sum_{i=1}^{n} \nabla F\left(x^{*}, \xi_{i}\right)^{T}\left(\phi_{i}^{k}-x^{*}\right)\right] \\
& +\left(\frac{1}{\kappa}-\gamma \mu\right) c\left\|x_{k}-x^{*}\right\|^{2} \\
& +c \gamma\left(\gamma(1+\beta)-\frac{1}{L}\right) \mathbb{E}\left(\left\|\nabla F\left(x_{k}, \xi_{j}\right)-\nabla F\left(x^{*}, \xi_{j}\right)\right\|^{2} \mid T^{k}\right) .
\end{aligned}
$$

Finalmente, substituindo os valores de $\gamma, c, \beta$ e $\kappa$ obtemos:

- $\frac{1}{n}-2 c \gamma \frac{L-\mu}{L}-2 c \gamma^{2} \beta \mu=\frac{1}{n}-2 \gamma \frac{1}{2 \gamma(1-\gamma \mu) n} \frac{L-\mu}{L}-2 \frac{1}{2 \gamma(1-\gamma \mu) n} \gamma^{2} \beta \mu$

$$
\begin{aligned}
& =\frac{1}{n}-\frac{1}{(1-\gamma \mu) n} \frac{L-\mu}{L}-\frac{1}{(1-\gamma \mu) n} \gamma \beta \mu \\
& =\frac{1}{n}-\frac{1}{(1-\gamma \mu) n} \frac{L-\mu}{L}-\frac{1}{(1-\gamma \mu) n} \gamma \frac{2 \mu n+L}{L} \mu \\
& =\frac{1}{n}-\frac{1}{(1-\gamma \mu) n L}(L-\mu+\gamma \mu(2 \mu n+L)) \\
& =\frac{1}{n}-\frac{1}{\left(1-\frac{1}{2(\mu n+L)} \mu\right) n L}\left(L-\mu+\frac{1}{2(\mu n+L)} \mu(2 \mu n+L)\right) \\
& =\frac{1}{n}-\frac{2(\mu n+L)}{(2(\mu n+L)-\mu) n L}\left(\frac{2 \mu n L+2 L^{2}-2 \mu^{2} n-2 \mu L+2 \mu^{2} n+\mu L}{2(\mu n+L)}\right) \\
& =\frac{1}{n}-\frac{2 \mu n L+2 L^{2}-\mu L}{(2(\mu n+L)-\mu) n L}=\frac{1}{n}-\frac{(2(\mu n+L)-\mu) L}{(2(\mu n+L)-\mu) n L} \\
& =\frac{1}{n}-\frac{1}{n}=0 .
\end{aligned}
$$

- $\frac{1}{\kappa}+2 c \gamma^{2}\left(1+\beta^{-1}\right) L-\frac{1}{n}$ : vejamos primeiro $1+\beta^{-1}$,

$$
\begin{aligned}
1+\beta^{-1} & =1+\frac{L}{2 \mu n+L}=\frac{2 \mu n+L+L}{2 \mu n+L} \\
& =\frac{2(\mu n+L)}{2 \mu n+L}=\frac{1}{\gamma(2 \mu n+L)} .
\end{aligned}
$$


Agora, substituindo os valores de $1+\beta^{-1}, c$ e $\kappa$ obtemos:

$$
\begin{aligned}
\frac{1}{\kappa}+2 c \gamma^{2}\left(1+\beta^{-1}\right) L-\frac{1}{n} & =\gamma \mu+2 \frac{1}{2 \gamma(1-\gamma \mu) n} \frac{1}{\gamma(2 \mu n+L)} \gamma^{2} L-\frac{1}{n} \\
& =\gamma \mu+\frac{1}{(1-\gamma \mu) n}\left(\frac{L}{2 \mu n+L}\right)-\frac{1}{n} \\
& =\frac{L+n(1-\gamma \mu)(2 \mu n+L)\left(\gamma \mu-\frac{1}{n}\right)}{n(1-\gamma \mu)(2 \mu n+L)} \\
& =\frac{L+(1-\gamma \mu)(2 \mu n+L)(n \gamma \mu-1)}{n(1-\gamma \mu)(2 \mu n+L)} .
\end{aligned}
$$

Olhando separadamente numerador e denominador obtemos:

$-L+(1-\gamma \mu)(2 \mu n+L)(n \gamma \mu-1)=L+n \mu \gamma(1-\gamma \mu)(2 \mu n+L)-(1-\gamma \mu)(2 \mu n+L)$

$$
\begin{aligned}
& =L+n \mu \gamma(2 \mu n+L)-n \mu^{2} \gamma^{2}(2 \mu n+L)-(1-\gamma \mu)(2 \mu n+L) \\
& \leq L+n \mu \gamma(2 \mu n+L)-(1-\gamma \mu)(2 \mu n+L) \\
& =n \mu \gamma(2 \mu n+L)+\gamma \mu(2 \mu n+L)-2 \mu n .
\end{aligned}
$$

Agora, como $\gamma(2 \mu n+L)=\frac{2 \mu n+L}{2 \mu n+2 L} \leq 1$ temos:

$$
L+(1-\gamma \mu)(2 \mu n+L)(n \gamma \mu-1) \leq \mu n+\mu-2 \mu n=\mu(1-n) .
$$

- Para demonstrar que o denominador é maior ou igual a 0 basta com provar que $(1-\gamma \mu) \geq$ 0

$$
1-\gamma \mu=1-\frac{\mu}{2 \mu n+L}=2 \mu n+L-\mu=\mu(2 n-1)+L \geq 0 .
$$

Voltando a (2.45) obtemos:

$$
\frac{1}{\kappa}+2 c \gamma^{2}\left(1+\beta^{-1}\right) L-\frac{1}{n} \leq \frac{\mu(1-n)}{\mu(2 n-1)+L} \leq 0 .
$$

- $\frac{1}{\kappa}-\gamma \mu=\gamma \mu-\gamma \mu=0$.

- $c \gamma\left(\gamma(1+\beta)-\frac{1}{L}\right)=c \gamma\left(\frac{1}{2(\mu n+L)}\left(1+\frac{2 \mu n+L}{L}\right)-\frac{1}{L}\right)$

$$
\begin{aligned}
& =c \gamma\left(\frac{1}{2(\mu n+L)}\left(2 \frac{\mu n+L}{L}\right)-\frac{1}{L}\right) \\
& =c \gamma\left(\frac{1}{L}-\frac{1}{L}\right)=0 .
\end{aligned}
$$

Então,

$$
\begin{aligned}
\mathbb{E}\left(T^{k+1} \mid T^{k}\right)-T^{k} & \leq-\frac{1}{\kappa} T^{k}+ \\
& +\left(\frac{1}{\kappa}+2 c \gamma^{2}\left(1+\beta^{-1}\right) L-\frac{1}{n}\right)\left[\frac{1}{n} \sum_{i=1}^{n} F\left(\phi_{i}^{k}, \xi_{i}\right)-f\left(x^{*}\right)-\frac{1}{n} \sum_{i=1}^{n} \nabla F\left(x^{*}, \xi_{i}\right)^{T}\left(\phi_{i}^{k}-x^{*}\right)\right] \\
& \leq-\frac{1}{\kappa} T^{k} .
\end{aligned}
$$

Pois, por convexidade de F, $\frac{1}{n} \sum_{i=1}^{n} F\left(\phi_{i}^{k}, \xi_{i}\right)-f\left(x^{*}\right)-\frac{1}{n} \sum_{i=1}^{n} \nabla F\left(x^{*}, \xi_{i}\right)^{T}\left(\phi_{i}^{k}-x^{*}\right)>0$. Assim, concluimos que

$$
\mathbb{E}\left(T^{k+1} \mid T^{k}\right)-T^{k} \leq-\frac{1}{\kappa} T^{k} .
$$


Corolário 2.3.1. [DBLJ14]

$\mathbb{E}\left(\left\|x_{k}-x^{*}\right\|^{2}\right) \leq\left(1-\frac{\mu}{2(\mu n+L)}\right)^{k}\left[\left\|x_{0}-x^{*}\right\|^{2}+\frac{\mu}{\mu n+L}\left(f\left(x_{0}\right)-\nabla f\left(x^{*}\right)^{T}\left(x_{0}-x^{*}\right)-f\left(x^{*}\right)\right)\right]$

onde o valor esperado é tomado sobre todas as possiveis escolhas do indice $j$ até a iteração $k$.

Demonstração. Pela Proposição 2.3.5 temos que

$$
\frac{1}{n} \sum_{i=1}^{n} F\left(\phi_{i}^{k}, \xi_{i}\right)-f\left(x^{*}\right)-\frac{1}{n} \sum_{i=1}^{n} \nabla F\left(x^{*}, \xi_{i}\right)^{T}\left(\phi_{i}^{k}-x^{*}\right) \geq 0,
$$

assim, pela definição de $T^{k}$ sabemos que $c\left\|x_{k}-x^{*}\right\|^{2} \leq T^{k}$. Calculando o valor esperado condicional em $T^{k-1}$ no resultado do Teorema 2.3.2 e lembrando que $\phi_{i}^{0}=x^{0}$ obtemos:

$$
\begin{aligned}
c \mathbb{E}\left(\left\|x_{k}-x^{*}\right\|^{2} \mid T^{k-1}\right) & \leq \mathbb{E}\left(T^{k} \mid T^{k-1}\right) \leq\left(1-\frac{1}{\kappa}\right) \mathbb{E}\left(T^{k-1} \mid T^{k-1}\right) \\
& =\left(1-\frac{1}{\kappa}\right)^{k} \mathbb{E}\left(T^{0} \mid T^{k-1}\right) \\
& =\left(1-\frac{1}{\kappa}\right)^{k} T^{0} \\
& =\left(1-\frac{1}{\kappa}\right)^{k}\left[\frac{1}{n} \sum_{i} F\left(\phi_{i}^{0}, \xi_{i}\right)-f\left(x^{*}\right)-\frac{1}{n} \sum_{i} \nabla F\left(x^{*}, \xi_{i}\right)^{T}\left(\phi_{i}^{0}-x^{*}\right)+c\left\|x_{0}-x^{*}\right\|^{2}\right] \\
& =\left(1-\frac{1}{\kappa}\right)^{k}\left[f\left(x^{0}\right)-f\left(x^{*}\right)-\nabla f\left(x^{*}\right)^{T}\left(x^{0}-x^{*}\right)+c\left\|x_{0}-x^{*}\right\|^{2}\right]
\end{aligned}
$$

Substituindo os valores de $\kappa, \gamma$ e $c$ obtemos:

$$
\begin{aligned}
\mathbb{E}\left(\left\|x_{k}-x^{*}\right\|^{2} \mid T^{k-1}\right) & \leq(1-\gamma \mu)^{k} \frac{1}{c}\left[f\left(x^{0}\right)-f\left(x^{*}\right)-\nabla f\left(x^{*}\right)^{T}\left(x^{0}-x^{*}\right)+c\left\|x_{0}-x^{*}\right\|^{2}\right] \\
& =\left(1-\frac{\mu}{2(\mu n+L)}\right)^{k}\left[\frac{1}{c}\left(f\left(x^{0}\right)-f\left(x^{*}\right)-\nabla f\left(x^{*}\right)^{T}\left(x^{0}-x^{*}\right)\right)+\left\|x_{0}-x^{*}\right\|^{2}\right]
\end{aligned}
$$

Calculando o valor de $\frac{1}{c}$ :

$$
\begin{aligned}
\frac{1}{c} & =2 \gamma(1-\gamma \mu) n=2 \frac{1}{2(\mu n+L)}\left(1-\frac{\mu}{2(\mu n+L)}\right) n \\
& =\frac{1}{(\mu n+L)}\left(\frac{2(\mu n+L)-\mu}{2(\mu n+L)}\right) n \\
& =\frac{n(2 \mu(n-0.5)+2 L)}{2(\mu n+L)^{2}} \\
& \leq \frac{n(2 \mu n+2 L)}{2(\mu n+L)^{2}} \\
& =\frac{n}{(\mu n+L)} .
\end{aligned}
$$

Finalmente, voltando em 2.48, 


$$
\mathbb{E}\left(\left\|x_{k}-x^{*}\right\|^{2} \mid T^{k-1}\right) \leq\left(1-\frac{\mu}{2(\mu n+L)}\right)^{k}\left[\frac{n}{(\mu n+L)}\left(f\left(x^{0}\right)-f\left(x^{*}\right)-\nabla f\left(x^{*}\right)^{T}\left(x^{0}-x^{*}\right)\right)+\left\|x_{0}-x^{*}\right\|^{2}\right]
$$




\section{Capítulo 3}

\section{Resultados}

Nesta seção se apresentam dois exemplo para analisar o desempenho das metodologias antes descritas. Especificamente, o Algoritmo 2.1.2 - SGD, os algoritmos de tamanho amostral variável (Algoritmo 2.2.1 - DSSS e Algoritmo 2.2.2 - LSSS) e as metodologías pertencentes ao grupo de redução da variância (Algoritmo 2.3.1 - SVRG e Algoritmo 2.3.2 - SAGA).

Em ambos casos estudados, os dados correspondem a problemas de classificação. O primeiro deles, é uma base de dados simulada, no qual se quer classificar os mesmos em duas categorias. Por outro lado, o segundo pertence a uma base de dígitos da vida real.

Ao longo do capítulo, Erro de classificação fala a respeito da porcentagem de dados na qual a resposta prevista pelos algoritmos é diferente da real. Estabeleceremos como medida de desempenho a quantidade de vezes que cada algoritmo executa a operação de calcular

$$
\frac{\partial F\left(x, \xi_{i}\right)}{\partial x}
$$

a qual foi escolhida pelo fato de ser a operação que acontece no cálculo do gradiente das aproximações da função objetivo em todos os algoritmos.

\subsection{Motivação para o problema de classificação}

Os dois exemplos que serão apresentados neste capítulo pertencem a problemas de classificação. Em ambos a função de aproximação é a função sigmoidal, que para o caso da base de dígitos, é uma generalização da Regressão Logística conhecida como Regressão Multinomial. Nesta seção se explica a origem do uso desta função como também a derivação da função de perda que usar-se-á ao longo dos testes. A teoria definida a seguir pode ser analisada com detalhe em [AMMIL12].

No problema de classificação, estamos interessados em encontrar um hiperplano que separe o conjunto de dados em $K \geq 2$ categorias. Inicialmente, consideremos o caso mais simples de $K=2$. Sejam os pares $\left\{\left(\xi_{i}, y_{i}\right)\right\}_{i=1}^{N} \operatorname{com} \xi_{i} \in \mathbb{R}^{d}$ e $y_{i} \in\{-1,1\} \forall i$, onde $y_{i}=1$ se $\xi_{i}$ pertence à categoria 1 , e $y_{i}=-1$ caso contrário. Queremos encontrar $\left(x_{0}, x\right) \in \mathbb{R}^{d+1}$ para o qual o hiperplano $h(\xi)=\operatorname{sign}\left(x^{T} \xi+x_{0}\right)$ classifica corretamente os dados, isto é, queremos encontrar a solução do seguinte problema de minimização:

$$
\min _{\left(x_{0}, x\right) \in \mathbb{R}^{d+1}} \frac{1}{N} \sum_{i=1}^{N} \mathbb{1}\left(y_{i} \neq \operatorname{sign}\left(x^{T} \xi+x_{0}\right)\right)
$$

Logo, a resolução de (3.1) é NP-difícil pois $\operatorname{sign}(\cdot)$ e $\mathbb{1}(\cdot)$ são funções com discontinuidade (como pode-se ver na Figura 3.1) e inteiras, derivando assim um problema combinatório.

Uma possível alternativa para a resolução de (3.1) é encontrar uma formulação similar continua. Consideremos o seguinte problema de minimização: 


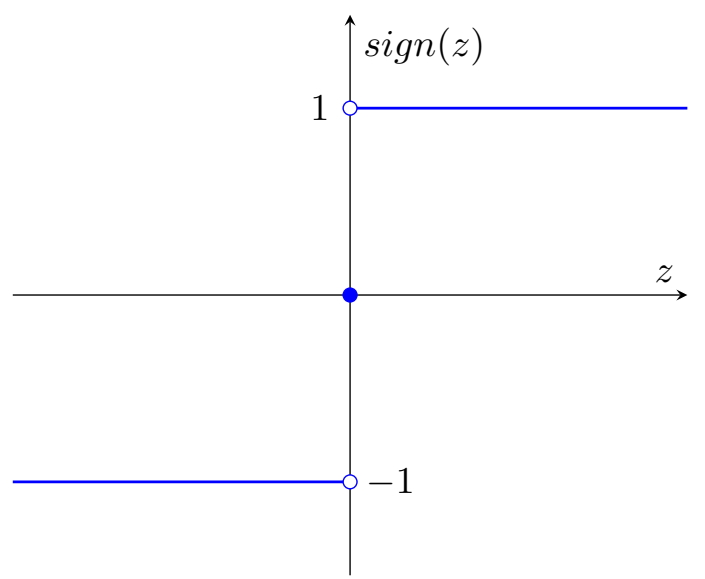

Figura 3.1: Gráfico da função $\operatorname{sign}(z)$.

$$
\min _{\left(x_{0}, x\right) \in \mathbb{R}^{d+1}} \frac{1}{N}\left(\sum_{i \in I} \max \left\{0, x^{T} \xi_{i}+x_{0}\right\}^{2}+\sum_{i \in O} \max \left\{0,-\left(x^{T} \xi_{i}+x_{0}\right)\right\}^{2}\right)
$$

onde $I=\left\{i \in\{1, \ldots, N\} \mid y_{i}=-1\right\}$ e $O=\left\{i \in\{1, \ldots, N\} \mid y_{i}=1\right\}$. Pode-se observar que para todos os $i \in I$ que não estão sendo classificados corretamente temos que $x^{T} \xi_{i}+x_{0}>0$, e assim o $\max \left\{0, x^{T} \xi_{i}+x_{0}\right\}=x^{T} \xi_{i}+x_{0}$. Para o caso de $i \in O$ aconteceria de manera equivalente obtendo que $\max \left\{0,-\left(x^{T} \xi_{i}+x_{0}\right)\right\}=x^{T} \xi_{i}+x_{0}$. Finalmente, encontrar a solução do problema (3.2) é igual a minimizar todos os valores do hiperplano $x^{T} \xi_{i}+x_{0}$ para os $i \in\{1, \ldots, N\}$ mal classificados. Assim, o problema (3.2) é equivalente a (3.1).

Consideremos agora otra possível formulação contínua do problema de classifcação (3.1). Seja a função sigmoidal $\varphi(\xi)$ definida como $\varphi(\xi)=P\left(y_{i}=1 \mid \xi_{i}\right)=\frac{1}{1+\exp \left\{-\left(x^{T} \xi+x_{0}\right)\right\}}$. Assim,

$$
P\left(y_{i} \mid \xi_{i}\right)= \begin{cases}\varphi(\xi) & \text { se } y_{i}=1 \\ 1-\varphi(\xi) & \text { se } y_{i}=-1\end{cases}
$$

Pode-se observar que podemos reescrever $\varphi(\xi)$ como $\frac{1}{1+\exp \left\{-y_{i}\left(x^{T} \xi+x_{0}\right)\right\}}$, pois,

$$
\begin{aligned}
1-\frac{1}{1+\exp \left\{-\left(x^{T} \xi+x_{0}\right)\right\}} & =\frac{\exp \left\{-\left(x^{T} \xi+x_{0}\right)\right.}{1+\exp \left\{-\left(x^{T} \xi+x_{0}\right)\right\}} \\
& =\frac{1}{1+\exp \left\{\left(x^{T} \xi+x_{0}\right)\right\}}
\end{aligned}
$$

Se supomos que todos os $\left\{\left(\xi_{i}, y_{i}\right)\right\}_{i=1}^{N}$ são $i . i . d$ então a probabilidade de obter todos os valores de $y_{i}$ seria igual ao produto de todas as probabilidades $\left(P\left(y_{1} \mid \xi_{1}\right)\right.$ e $\left.P\left(y_{2} \mid \xi_{2}\right), \ldots, P\left(y_{n} \mid \xi_{n}\right)\right)$, isto é, $P(y \mid \xi)=\prod_{i=1}^{N} P\left(y_{i} \mid \xi_{i}\right)$. Assim, maximizando este produto, resolvemos o problema de aproximação das probabilidades de pertencer a cada categoria. Logo, a maximização de $P(y \mid \xi)$ é equivalente a minimizar o $-\ln (P(y \mid \xi))$. Reescrevendo substituindo os valores de $\varphi$ e multiplicando pela constante 
$\frac{1}{N}$ obtemos:

$$
\begin{aligned}
-\frac{1}{N} \ln (P(y \mid \xi)) & =-\frac{1}{N} \ln \left(\prod_{i=1}^{N} P\left(y_{i} \mid \xi_{i}\right)\right) \\
& =\frac{1}{N} \sum_{i=1}^{N} \ln \left(\frac{1}{P\left(y_{i} \mid \xi_{i}\right)}\right) \\
& =\frac{1}{N} \sum_{i=1}^{N} \ln \left(1+\exp \left\{-y_{i}\left(x^{T} \xi+x_{0}\right)\right\}\right) .
\end{aligned}
$$

Podemos ver que na medida em que aumentamos $y_{i}\left(x^{T} \xi+x_{0}\right)$ o valor da nossa função objetivo diminiu, o que corresponde a classificações corretas equivalentes às obtidas pelo hiperplano $\operatorname{sign}\left(\xi_{i}\right)$. Assim, finalmente vinculamos o nosso problema inicial de classificação com a construção da regressão logística. Agora, (3.3) pode ser reescrita como,

$$
\frac{1}{N} \sum_{i=1}^{N} \ln \left(1+\exp \left\{-y_{i}\left(x^{T} \xi+x_{0}\right)\right\}\right)=\frac{1}{N} \sum_{i=1}^{N} \ln \left(1+\exp \left\{y_{i}\left(x^{T} \xi+x_{0}\right)\right\}\right)-y_{i}\left(x^{T} \xi+x_{0}\right) .
$$

Por último, uma vantagem da minimição da função de erro (3.4) sobre a resolução do problema (3.2) é a propriedade de convexidade, que garante a existência de um minimizador único, pois tanto a função linear como o $\ln \left(1+\exp \left\{y_{i}\left(x^{T} \xi+x_{0}\right)\right\}\right)$ são convexas (a Hessiana (3.5) do $\ln \left(1+\exp \left\{y_{i}\left(x^{T} \xi+x_{0}\right)\right\}\right)$ é positiva semi-definida). Desse modo, a formulação obtida por meio da função sigmoidal é a mais utilizada em problemas de classificação, sendo escolhida assim com a função que será minimizada ao longo deste capítulo.

$$
\begin{aligned}
\frac{\partial^{2} \ln \left(1+\exp \left\{y_{i}\left(x^{T} \xi_{i}\right)\right\}\right)}{\partial x_{k} \partial x_{j}} & =\frac{\partial}{\partial x_{k}}\left(\left(1-\frac{1}{1+\exp \left\{y_{i}\left(x^{T} \xi_{i}\right)\right\}}\right) y_{i} \xi_{i}^{(j)}\right) \\
& =y_{i}^{2} \xi_{i}^{(j)} \xi_{i}^{(k)} \frac{\exp \left\{y_{i}\left(x^{T} \xi_{i}\right)\right\}}{\left(1+\exp \left\{y_{i}\left(x^{T} \xi_{i}\right)\right\}\right)^{2}} \geq 0 \forall k, j .
\end{aligned}
$$

\subsection{Mistura de Gaussianas}

O primeiro estudo realizado corresponde a uma base de dados gerada por meio de uma simulação como é explicado pelos autores Hastie, T.,Tibshirani, R. e Friedman, J. na referência [HTF08]. Seja $\xi \in \mathbb{R}^{2}$ uma variável aletória com distribuição normal definida por $\mathcal{N}(\mu, \boldsymbol{\Sigma})$ com

$$
\boldsymbol{\Sigma}=\left[\begin{array}{cc}
1 / 5 & 0 \\
0 & 1 / 5
\end{array}\right]
$$

e $\mu \sim \mathcal{N}\left(\mu_{1}, \mathbf{I}\right)$ onde $\mu_{\mathbf{1}}= \begin{cases}(0,1)^{T} & \text { se } \xi \text { pertence à clase laranja } \\ (1,0)^{T} & \text { se } \xi \text { pertence à clase azul }\end{cases}$ 


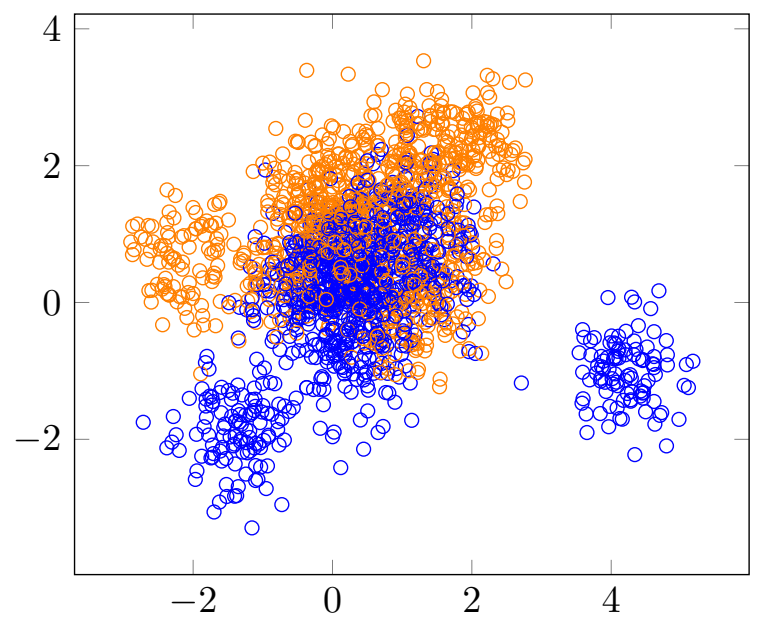

Figura 3.2: Exemplo da amostra no problema de mistura de Gaussianas

Uma alternativa para a função de aproximação do hiperplano de classificação é uma rede neural. Esta função nasce da tentativa de simular computacionalmente a forma em que o nosso cérebro aprende. De uma forma simples, recebemos um estímulo (vetor de entrada), os neurônios se conectam entre eles e geram uma resposta final para esse estímulo. No caso de classificação, poderia ser por exemplo reconhecer uma imagem. Desta forma, foram criadas estruturas computacionais, chamadas também de neurônios, que processam os dados de entrada (ou as saídas de otros neurônios, dependendo da localização do neurônio na rede neural), por medio de uma soma ponderada e trasformando-os com uma função matemática conhecida como "Função de ativação". No caso que será apresentado neste trabalho, supomos que a função de ativação é diferenciável. Cada conexão possue um pesso (sináptico) e é por isto que podemos representar a saída de um neurônio $j$ com função de ativação $\varphi$ como $f_{j}=\varphi\left(x_{1} z_{1}+\ldots+x_{k} z_{k}\right)$ onde cada $x_{i}$ representa o peso sináptico entre $z_{i}$ e o neurônio $j$.

Assim, uma rede neural pode-se definir como um conjunto de neurônios distribuidos em diferentes camadas de representações, cada uma com uma função de ativação definida. Cada camada tem a característica de conectividade com a camada anterior e posterior (caso não seja a camada de saída). Na Figura 3.3 pode-se observar uma estrutura de uma rede neural de múltiplas camadas, neste caso com uma camada oculta de $k$ neurônios e uma saída com $s$ neurônios. De manera geral, pode-se considerar uma rede neural como uma composição de funções. Para maior aprofundamento na teoria de Redes Neurais, consultar [Hay05, AMMIL12].

Voltando ao problema de classificação, foi definida como função de aproximação uma rede neural com 10 neurônios na camada oculta e um único neurônio na camada de saída, ambas camadas com função de ativação sigmoidal. Estamos interessados na minimização do erro definido por:

$$
\min _{x \in \mathbb{R}^{d}} \mathbb{E}\left(\ln \left(1+e^{x_{s}^{T} f_{o}}\right)-y x_{s}^{T} f_{o}\right)+\frac{\lambda}{2}\|x\|_{2}^{2}
$$

onde $x=\left(x_{o}, x_{s}\right)$ corresponde aos pesos da camada oculta e de saída respectivamente, $f_{o}=\frac{1}{1+e^{-x_{O}^{T} \xi}}$ saídas obtidas na camada oculta, $d=41$ total de pesos sinâpticos presentes na rede e $\lambda=0.2 \mathrm{o}$ parâmetro de regularização. Se gerou uma amostra para a estimativa da solução igual a 2,000 (base de treinamento) e uma adicional para monitorar o desempenho da minimização de 858 dados (base de validação). Adicionalmente, para analisar o poder de generalização da solução encontrada, se avaliou num total de 10,000 novos dados (base de teste).

Definindo inicialmente os parâmetros necessários para cada algoritmo, foram testados para cada um deles uma serie de possíveis valores, especificamente,

- Para o Algoritmo 2.1.2 - SGD na seleção do exponente $\alpha$ do tamanho do passo na atualização do $x$ foram testados os valores $\alpha \in\{0.6,0.65,0.7,0.75,0.8,0.85,0.9,0.95,1\}$. Na Figura 3.4 


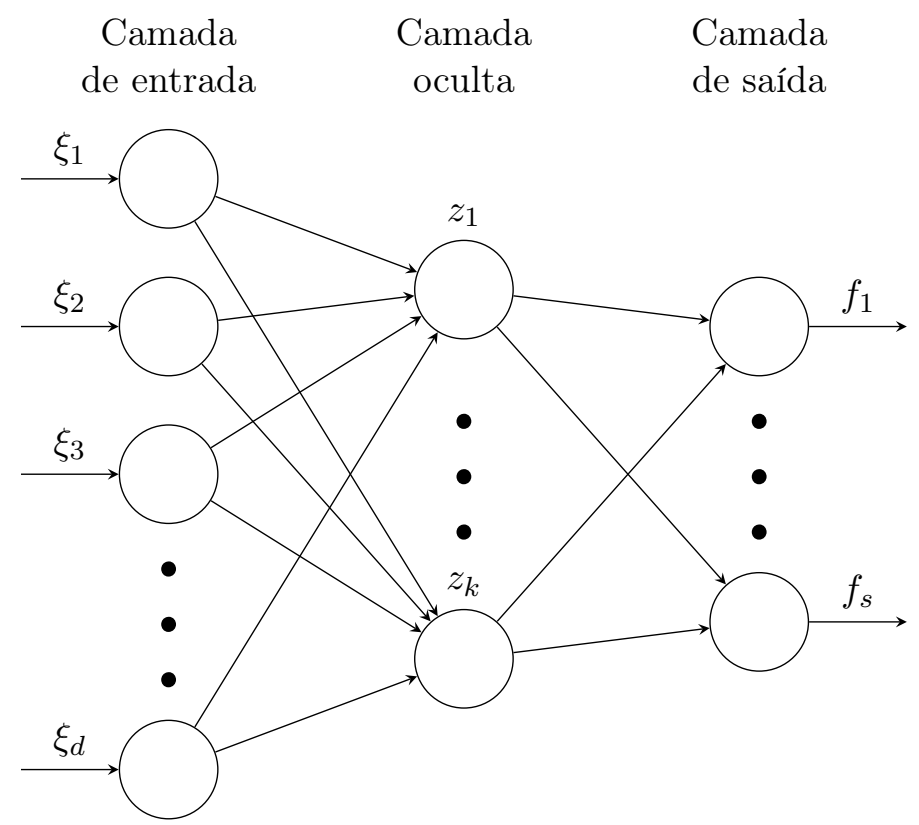

Figura 3.3: Estrutura de uma rede neural de múltiplas camadas

pode-se observar a cada 100 iterações, a diminuição da função objetivo. A medida que aumentamos o $\alpha$ o decrescimento é mais demorado.

- Para o Algoritmo 2.3.1 - SVRG se estipularam os parâmetros $\eta$ do tamanho da atualização do passo e $m$, o total de iterações a partir da qual se atualiza o gradiente no total dos dados (ver Passo 55.2 no Algoritmo 2.3.1 - SVRG). Foram testados, por um total de 200,000 iterações os valores $\eta \in\{0.002,0.003,0.004,0.005,0.01,0.02,0.03,0.04,0.05,0.1,0.2,0.3\} \mathrm{e}$ $m \in\{100,200,300,500,750,1000\}$ selecionando $\eta 0.01$ e $m 200$, parâmetros que encontraram o menor erro na amostra de validação igual a $27.62 \%$.

- No Algoritmo 2.3.2 - SAGA foi escolhido o parâmetro $\gamma$ igual a 0.02 definido para a atualização do $x$ no Passo 5. O parâmetro atingiu um erro de classificação na amostra de validação de $27.62 \%$. Neste caso foram testados os valores $\gamma \in\{0.002,0.003,0.004,0.005,0.01,0.02,0.03$, $0.04,0.05,0.1,0.2,0.3\}$ por um total de 200,000 iterações.

Foram geradas as soluções do problema de minimização considerando como critério de parada o máximo de iterações igual a 200,000, ou, para todos os algoritmos excepto o Algoritmo 2.1.2 - SGD, $\left\|x_{k}-x_{k-1}\right\|<10^{-4}$. Na Figura 3.5 observa-se o avanço do erro a medida que o custo acumulado do algoritmo aumenta. Especificamente, o Algoritmo 2.1.2 - SGD e Algoritmo 2.3.2 - SAGA, pela natureza de ser estocástico e em cada iteração tomar um $\xi_{i}$ diferente para a minimização de (3.6), o erro nas iterações não decresce uniformemente, a diferença dos algoritmos de tamanho amostral variável (Algoritmo 2.2.1 - DSSS e Algoritmo 2.2.2 - LSSS) que, a medida que se aumenta o tamanho amostral da aproximação do valor esperado, o erro diminui. No entanto, os 5 algoritmos atingem erros muito próximos, o que gera que a comparação do desempenho se centre no custo de cada um, favorecendo assim a Stochastic Gradient Descent. 


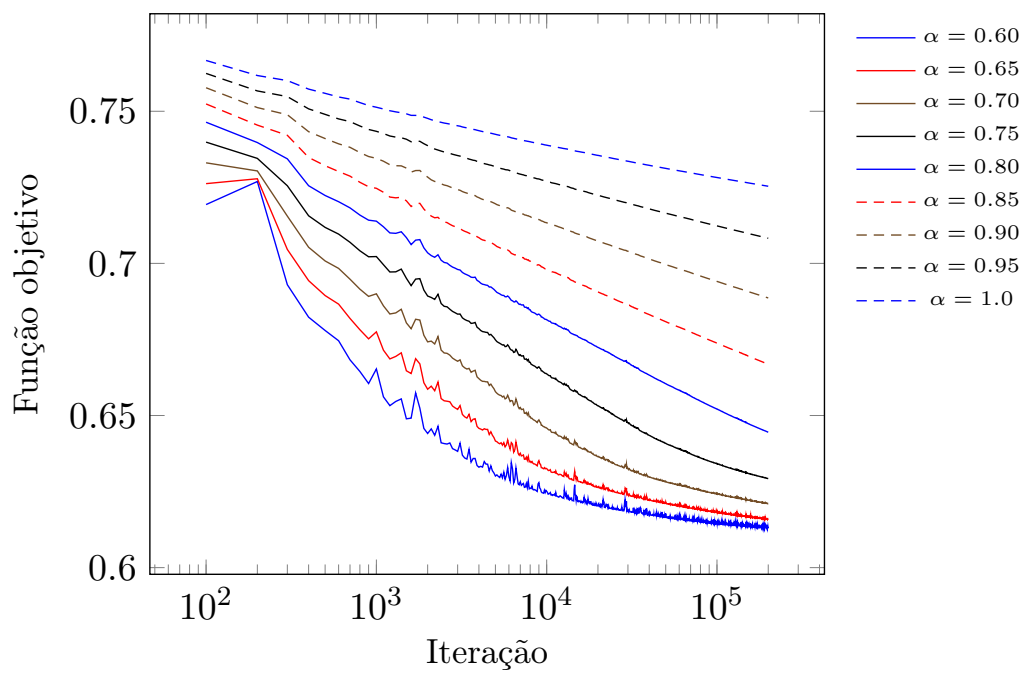

Figura 3.4: Erro de classificação nos dados de validação na seleção do parâmetro a no Algoritmo 2.1.2 $S G D$.

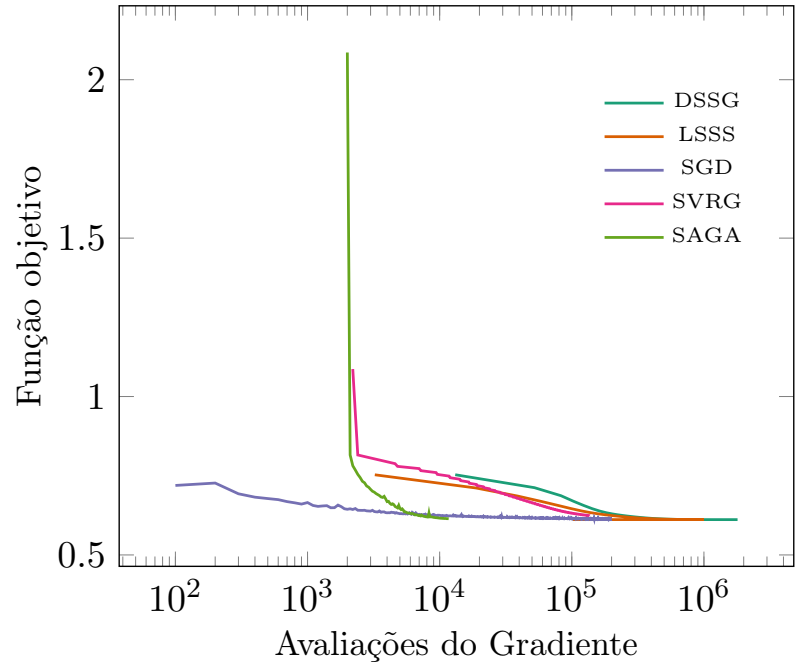

(a) Erro de aproximação em relação à quantidade de avaliações de gradiente.

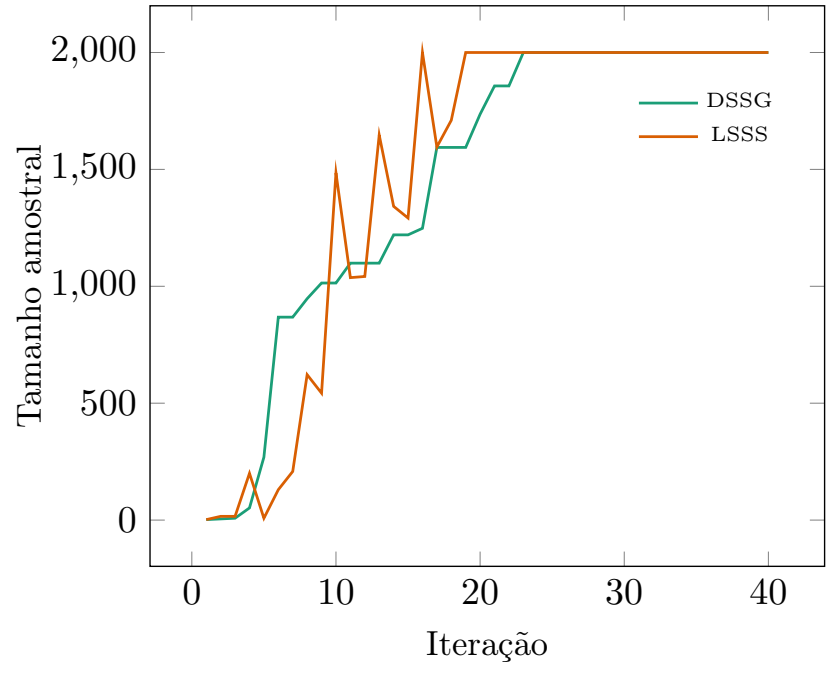

(b) Mudanças do tamanho amostral para os algoritmos de Sample Average Approximation.

Figura 3.5: Desempenho dos algoritmos no problema Mistura de Gaussianas.

Finalmente, avaliando o desempenho dos algoritmos na base de teste, pode-se observar que todos eles conseguem soluções que generalizam bem. Isto quer dizer que em nenhum dos 5 algoritmos se chega a soluções específicas para os dados de treinamento, se não, para qualquer conjunto de dados que possuam a mesma distribuição de probabilidades, a solução encontrada garante um erro de classificação pequeno (dada a natureza do problema).

\begin{tabular}{c|c|c|c|c|c}
\hline Base & SGD & SVRG & SAGA & DSSG & LSSS \\
\hline Treinamento & $27.15 \%$ & $27.35 \%$ & $27.10 \%$ & $27.25 \%$ & $27.25 \%$ \\
Validação & $27.97 \%$ & $27.62 \%$ & $27.62 \%$ & $27.86 \%$ & $27.86 \%$ \\
Teste & $26.78 \%$ & $26.93 \%$ & $26.33 \%$ & $26.58 \%$ & $26.58 \%$ \\
\hline
\end{tabular}

Tabela 3.1: Erro de classificação nas bases de treinamento, validação e teste para os dados de Mistura de Gaussianas. 


\section{$3.3 \quad$ MNIST}

A seguinte aplicação apresentada é um problema conhecido de classificação de imagens. Esta base, conhecida como MNIST (Modified National Institute of Standards and Technology) [LBBH98], corresponde a dígitos manuscritos entre 0 e 9, 50\% deles desenhados por empregados do Bureau do Census Americano e o 50\% restante por estudantes de ensino médio dos Estados Unidos, cada um corretamente clasificados, como pode-se observar na Tabela 3.2. Os dados estão divididos em três bases, treinamento, validação e teste que contêm 50.000, 10.000 e 10.000 imagens, respectivamente.

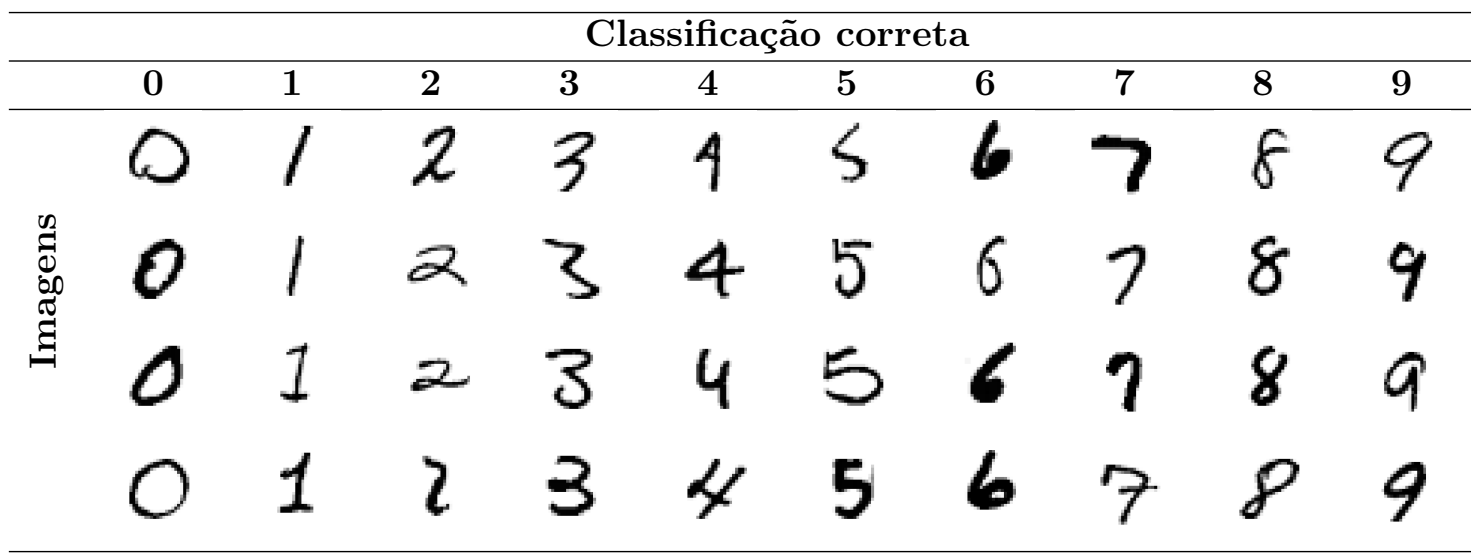

Tabela 3.2: Exemplo de dígitos corretamente classificados que fazem parte das amostras no problema MNIST

Seja $\left(\xi_{i}, y_{i}\right), \forall i \in\{1, \ldots, N\}$ o par de imagem e classificação, onde cada $\xi_{i} \in \mathbb{R}^{d}$ com $d=784$ (obtida pela representação vetorial da matriz de $28 \times 28$ pixels) e $y_{i} \in\{0, \ldots, 9\}$ é o valor da categoría correspondente. Neste problema, queremos minimizar o erro de classificação dado pela aproximação de uma regressão logística multinomial com regularização $\ell_{2}$, isto é,

$$
\min _{x \in \mathbb{R}^{m}} \mathbb{E}\left(-\ln \left(\frac{\exp \left(x^{T} \xi\right)}{\sum \exp \left(x^{T} \xi\right)}\right)\right)+\frac{\lambda}{2}\|x\|_{2}^{2}
$$

com $m=794$ o total de parâmetros a estimar (incluindo os interceptos).

\subsubsection{Seleção de hiperparâmetros}

Desde a seleção do problema a minimizar até os algoritmos de minimização, existem hiperparâmetros que devem ser especificados. Neste caso, para todos os seguintes resultados apresentados foi realizada a pré-seleção destes por meio de um grid, considerando os dados totais da base de treinamento (50.000 imagens) e avaliando o desempenho por meio do erro final nos dados de validação (10.000 imagens).

Primeiramente, foi selecionado o $\lambda$ da equação (3.7). Como o nosso objetivo é comparar os algoritmos descritos anteriormente, este parâmetro foi selecionado usando o algoritmo de Descida do Gradiente, pois queremos escolher o melhor $\lambda$ usando a maior quantidade de informação dos dados disponíveis. Além disso, como a escolha do $\lambda$ influi no resultado final de cada algoritmo, consideramos que esta seria a forma mais adequada para não dar vantagem a nenhum dos algoritmos a comparar. O algoritmo Descida do Gradiente não foi estudado neste trabalho devido a que a natureza dele é determinística. Ainda assim, na referência [eSW10] se encontra toda a teoría relacionada a este.

Considerando valores de $\lambda \in\left\{0,10^{-4}, 10^{-3}, 10^{-2}, 0.05,0.075,0.1,0.2,0.5,1\right\}$ foram registrados os erros cometidos nas amostras de treinamento e validação. Na Tabela 3.3 podemos observar como a medida que aumentamos o valor do $\lambda$ perdemos poder de discriminação em ambas bases, pois começamos a dar menos peso para a minimização do erro da estimativa entre a saída desejada 
e a saída obtida na regressão. Para $\lambda \in\left\{0,10^{-4}, 10^{-3}\right\}$ encontramos o menor erro na amostra de validação igual a $7.9 \%$. Selecionamos por tanto o maior $\lambda\left(10^{-3}\right)$ como o parâmetro de regularização da função a minimizar.

\begin{tabular}{c|c|c|c|c|c|c|c|c|c|c}
\hline & \multicolumn{10}{|c}{ Parâmetro de regularização } \\
\hline Base & $\mathbf{0}$ & $\mathbf{1 0}^{-\mathbf{4}}$ & $\mathbf{1 0}^{-\mathbf{3}}$ & $\mathbf{1 0}^{-\mathbf{2}}$ & $\mathbf{0 . 0 5}$ & $\mathbf{0 . 0 7 5}$ & $\mathbf{0 . 1}$ & $\mathbf{0 . 2}$ & $\mathbf{0 . 5}$ & $\mathbf{1}$ \\
\hline Treinamento & $8.2 \%$ & $8.2 \%$ & $8.2 \%$ & $18.1 \%$ & $19.9 \%$ & $24.9 \%$ & $23.7 \%$ & $23.9 \%$ & $32.8 \%$ & $32.9 \%$ \\
Validação & $7.9 \%$ & $7.9 \%$ & $\mathbf{7 . 9 \%}$ & $16.1 \%$ & $16.9 \%$ & $22.9 \%$ & $21.7 \%$ & $20.7 \%$ & $29.7 \%$ & $29.8 \%$ \\
\hline
\end{tabular}

Tabela 3.3: Erro de classificação nos dados de treinamento e validação na seleção do $\lambda$ no Problema (3.7)

Para o Algoritmo 2.1.2 - SGD devemos selecionar o parâmetro $\alpha$ no tamanho do passo $a_{k}=\frac{1}{k^{\alpha}}$ na atualização do $x_{k+1}$ no Passo 2. Foram testados os valores $\alpha \in(0.5,1]$ como pode-se observar na Tabela 3.4. Os menores valores de $\alpha$ são os que obtem os melhores resultados de aproximação (em 500,000 iterações), pois, pela definição do passo $a_{k}$, a medida que aumentamos o valor de $\alpha$, a atualização de $x_{k+1}$ é menor em menos iterações. Assim, continuando com o mesmo critério de seleção, foi escolhido $\alpha 0.59$ por obter o menor erro na amostra de validação.

\begin{tabular}{c|c|c}
\hline & \multicolumn{2}{|c}{ Base } \\
\hline$\alpha$ & Treinamento & Validação \\
\hline $\mathbf{0 . 5 5}$ & $8.18 \%$ & $7.83 \%$ \\
$\mathbf{0 . 5 6}$ & $8.24 \%$ & $7.88 \%$ \\
$\mathbf{0 . 5 7}$ & $8.26 \%$ & $7.84 \%$ \\
$\mathbf{0 . 5 8}$ & $8.27 \%$ & $7.89 \%$ \\
$\mathbf{0 . 5 9}$ & $8.34 \%$ & $\mathbf{7 . 8 2 \%}$ \\
$\mathbf{0 . 6 0}$ & $8.39 \%$ & $7.87 \%$ \\
$\mathbf{0 . 6 5}$ & $8.95 \%$ & $8.16 \%$ \\
$\mathbf{0 . 7 0}$ & $9.77 \%$ & $8.80 \%$ \\
$\mathbf{0 . 7 5}$ & $10.67 \%$ & $9.62 \%$ \\
$\mathbf{0 . 8 0}$ & $11.90 \%$ & $10.52 \%$ \\
$\mathbf{0 . 8 5}$ & $13.66 \%$ & $12.05 \%$ \\
$\mathbf{0 . 9 0}$ & $15.54 \%$ & $13.71 \%$ \\
$\mathbf{0 . 9 5}$ & $17.82 \%$ & $15.35 \%$ \\
$\mathbf{1}$ & $20.34 \%$ & $17.67 \%$ \\
\hline
\end{tabular}

Tabela 3.4: Erro de classificação nos dados de validação na seleção do parâmetro $\alpha$ de tamanho do passo no Algoritmo 2.1.2 - SGD.

No caso do Algoritmo 2.3.2 - SAGA deve-se selecionar o parâmetro $\gamma$ encontrado no Passo 5 na atualização do $x$. Foram testados os valores de $\gamma \in\left\{10^{-4}, 10^{-3}, 0.002,0.003,0.004,0.005,0.01,0.05,0.1\right\}$ por um máximo de 500,000 iterações. Na Tabela 3.5 pode-se observar as mudanças de erro obtidas, sinalizando que para os maiores e menores valores testados de $\gamma$, o erro na base de validação é maior. Seguindo a mesma análise descrita anteriormente, foi selecionado o $\gamma$ que gera o menor erro de classificação, o qual corresponde ao valor de 0.005 .

Para o Algoritmo 2.3.1 - SVRG precisamos determinar tanto o passo $\eta$ de atualização do $x_{k}$ como também o parâmetro $m$ de frequência de atualização do gradiente na base total. Foram considerados os valores de $\eta \in\{0.001,0.002,0.003,0.004,0.005,0.01,0.05,0.1\}$ e $m \in\{10,50,100$, $200,500,600,700,1000,2000,5000\}$. Dado que o total de combinações entre $\eta$ e $m$ é 80 , para tornar o experimento viável foi considerado um máximo de iterações de 50,000. Analisando a Figura 3.6 encontramos que o aumento da quantidade de iterações internas $m$ gera que menos vezes se atualize o gradiente global na base inteira com o $x_{k}$ mais recente, obtendo maiores erros de classificação que no caso de $m$ menores. Ao igual que no caso anterior, os extremos dos valores do passo $\eta$ 


\begin{tabular}{c|c|c|c|c|c|c|c|c|c}
\hline & \multicolumn{7}{|c}{ Parâmetro $\gamma$ de atualização do passo } \\
\hline Base & $\mathbf{1 0}^{-\mathbf{4}}$ & $\mathbf{1 0}^{-\mathbf{3}}$ & $\mathbf{0 . 0 0 2}$ & $\mathbf{0 . 0 0 3}$ & $\mathbf{0 . 0 0 4}$ & $\mathbf{0 . 0 0 5}$ & $\mathbf{0 . 0 1}$ & $\mathbf{0 . 0 5}$ & $\mathbf{0 . 1}$ \\
\hline Treinamento & $10.73 \%$ & $8.20 \%$ & $7.94 \%$ & $7.84 \%$ & $7.82 \%$ & $7.80 \%$ & $7.76 \%$ & $9.62 \%$ & $18.38 \%$ \\
Validação & $9.45 \%$ & $7.92 \%$ & $7.74 \%$ & $7.69 \%$ & $7.67 \%$ & $\mathbf{7 . 6 7 \%}$ & $7.69 \%$ & $9.54 \%$ & $16.97 \%$ \\
\hline
\end{tabular}

Tabela 3.5: Erro de classificação nos dados de validação na seleção do parâmetro $\eta$ no Algoritmo 2.3.2 $S A G A$.

geram convergências muito demoradas (passo pequeno) ou afastamento da possível solução ótima ocasionando maiores erros na aproximação (passos grandes). Finalmente, os valores escolhidos dado o menor erro foram $m 2000$ e $\eta 0.05$.

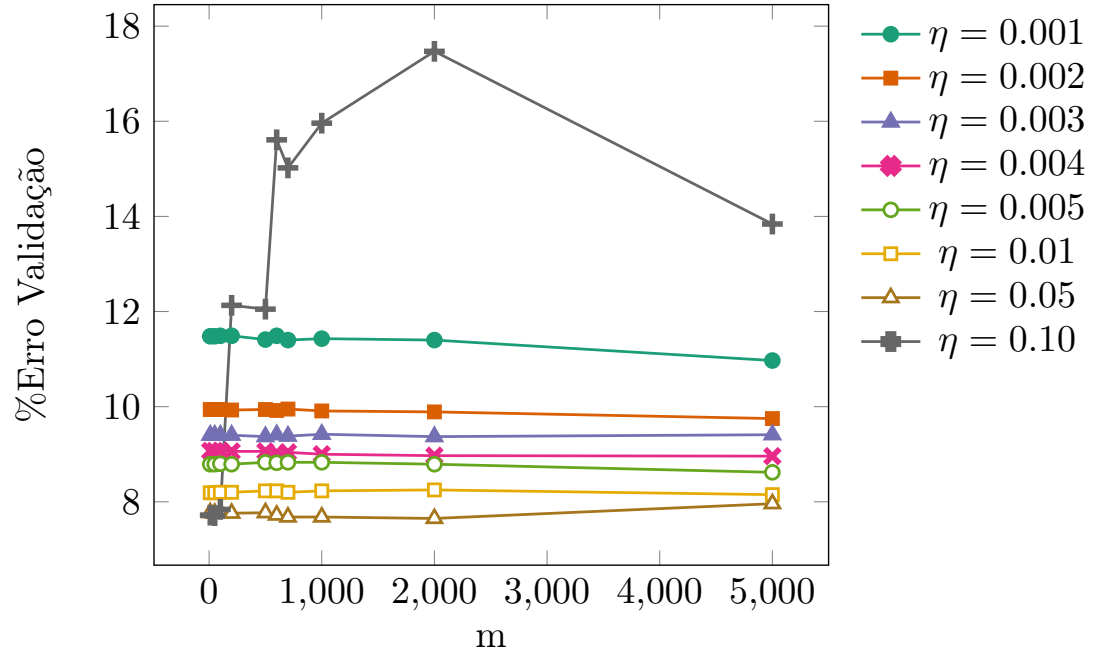

Figura 3.6: Erro de classificação nos dados de validação na seleção do parâmetro $\eta$ e m no Algoritmo 2.3.1 $S V R G$.

\subsubsection{Resultados finais}

Para realizar a comparação dos algoritmos, foram geradas as soluções para a base de treinamento por um número máximo de iterações igual a 500,000 ou por um tempo máximo de 2 dias. Uma condição de parada adicional para todos os algoritmo excepto para Stochastic Gradient Descent é $\left\|x_{k+1}-x_{k}\right\|^{2}<10^{-4}$. O único algoritmo que possue condições adicionais é o Algoritmo 2.2.2 - LSSS que foram apresentadas com detalhe na Seção 2.2.

Como foi comentado na Seção 2.3, para o Algoritmo 2.3.2 - SAGA deve-se guardar uma matriz de tamanho $N \times d$ para armazenar $\phi$ que corresponde ao gradiente no último $x_{k}$ onde cada $i \in$ $\{1, \ldots, N\}$ foi selecionado. Neste caso específico em que a função que estamos usando para aproximar a variável resposta é uma logística multinomial, o gradiente é equivalente ao produto interno entre as probabilidades de cada classe e o $\xi$, isto é,

$$
\begin{aligned}
\nabla \ell_{i} & =\nabla\left(-\log \left(\frac{\exp \left(x^{T} \xi_{i}\right)}{\sum \exp \left(x^{T} \xi_{i}\right)}\right)\right) \\
& =\nabla\left(-x_{y_{i}}^{T} \xi_{i}+\log \left(\sum \exp \left(x^{T} \xi_{i}\right)\right)\right) \\
& =-\mathbb{1}_{y_{i}=k} \xi_{i}+\frac{\exp \left(x^{T} \xi_{i}\right)}{\sum \exp \left(x^{T} \xi_{i}\right)} \xi_{i} \\
& =\xi_{i}\left(\frac{\exp \left(x^{T} \xi_{i}\right)}{\sum \exp \left(x^{T} \xi_{i}\right)}-\mathbb{1}_{y_{i}=k}\right)
\end{aligned}
$$


Assim, armazenando unicamente as probabilidades estimadas cada vez que o $\xi_{i}$ é selecionado, a dimensão de $\phi$ diminui a $N \times k$ onde $k$ é o total de clases do problema.

A cada 100 iterações foi avaliada a solução para as amostras de treinamento e validação e registrou-se tanto as avaliações do gradiente para uma amostra de tamanho 1 (i.e. $\nabla f_{i}$ ) como a função objetivo, medindo assim o avanço na diminuição do erro. No caso do Algoritmo 2.2.2 - LSSS e do Algoritmo 2.2.1 - DSSG , antes de atingir o tamanho amostral máximo, foram registrados a cada 10 iterações a função objetivo; uma vez que o $N_{k}=N_{\text {max }}$, foram guardados esses valores a cada 100 iterações. Esta decisão se deve ao fato de querer estudar o decrecimento do erro com tamanhos amostrais menores. Na Figura 3.7a podem-se observar os resultados obtidos para os 5 algoritmos estudados. O eixo Avaliações do gradiente encontra-se em escala logaritmica para facilitar a visualização. Para aquelas metodologias pertencentes ao grupo de tamanho amostral variável vemos que possuem o maior número de avaliações acumulado. Isto se deve ao fato de, como pode-se verificar na Figura 3.7b, antes da iteração 70 o tamanho amostral chegou (e estabilizou no caso de Line Search Variable Sample Size Selection) nas 50,000 imagens, assim, estes dois algoritmos terminam funcionando igual que Descida do Gradiente em poucas iterações. Para o Algoritmo 2.2.2 - LSSS pode-se observar que, pelo fato de atingir o máximo tamanho amostral nas primeiras iterações, as avaliações totais do gradiente aumentam significativamente comparadas com o Algoritmo 2.2.1 - DSSG que possue um crecimento constante (e sempre maior) no tamanho da amostra. Seria interessante estudar formas de evitar que nas primeiras iterações esse $N_{\max }$ seja alcançado.

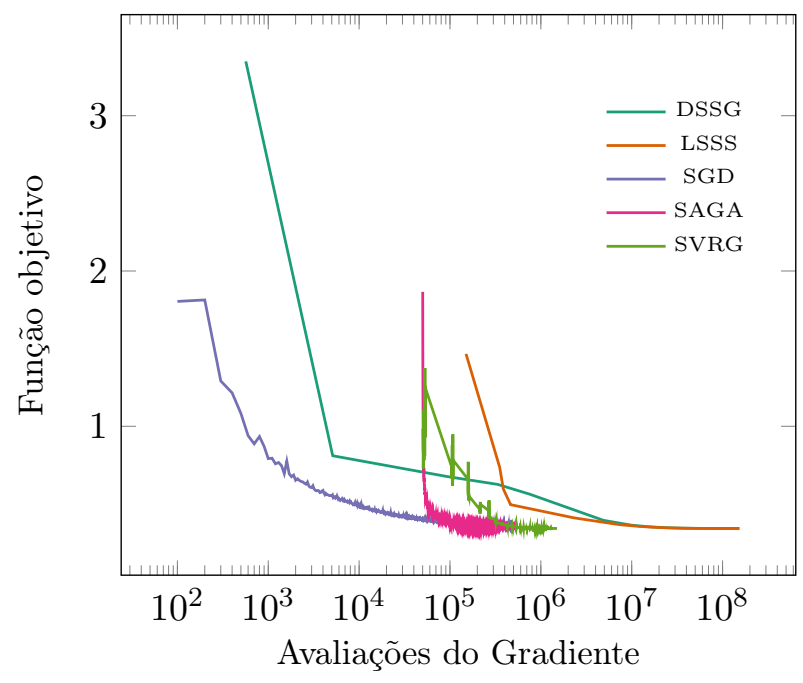

(a) Erro de aproximação em relação à quantidade de avaliações de gradiente.

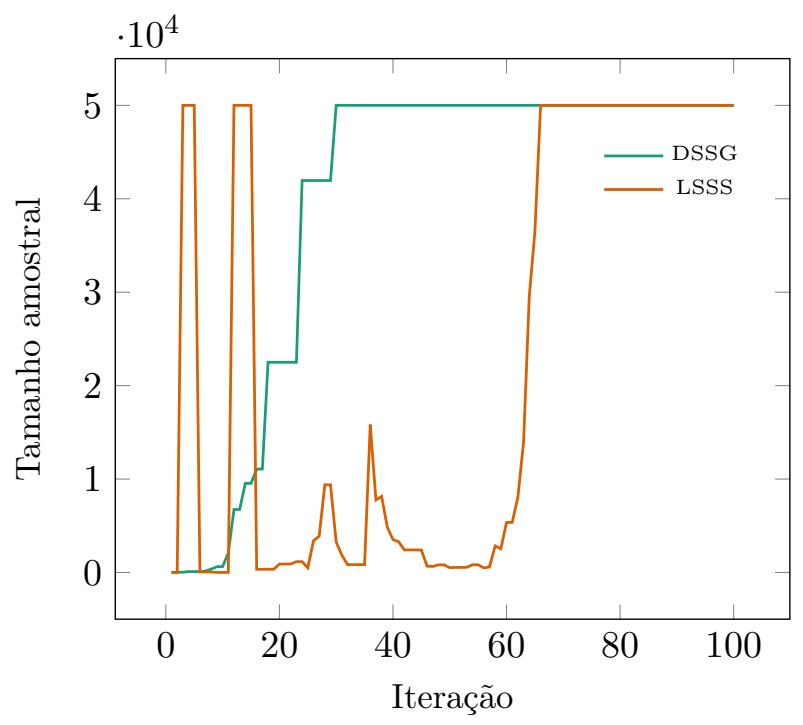

(b) Mudanças do tamanho amostral para os algoritmos de Sample Average Approximation.

Figura 3.7: Desempenho dos algoritmos no problema de MNIST.

Na Tabela 3.6 sumarizam-se os erros de classificação para as três bases. Stochastic Gradient Descent é o algoritmo que gera a pior aproximação, atingindo um erro de generalização de $8.22 \%$. Pelo contrário, as restantes 4 metodologias obtêm resultados similares entre elas, variando o erro de $7.80 \%$ até $7.81 \%$ na amostra de teste. Assim, complementando a análise prévia do gráfico, para este exemplo, os métodos de redução de variância são os que resultaram mais vantajosos observando o custo e o beneficio deles juntamente.

Finalmente, na Tabela 3.7 se observam alguns exemplos de imagens onde a classificação prevista pelos algoritmos resultou errada. Em todas as imagens apresentadas todos os algoritmos geram a mesma saída. No caso da última imagem correspondente ao dígito 9, nenhum dos algoritmos encontrou uma probabilidade estimada maior a 0.5 (o Algoritmo 2.1.2 - SGD obteve o maior valor igual a 0.20), isto indica que existem casos onde os dados apresentados não possuem caraterísticas em comum com outros da amostra, logrando assim praticamente uma classificação parecida à aleatória. 


\begin{tabular}{c|c|c|c|c|c}
\hline Base & SGD & SVRG & SAGA & DSSG & LSSS \\
\hline Treinamento & $8.34 \%$ & $7.79 \%$ & $7.79 \%$ & $7.78 \%$ & $7.78 \%$ \\
Validação & $7.82 \%$ & $7.67 \%$ & $7.66 \%$ & $7.64 \%$ & $7.65 \%$ \\
Teste & $8.22 \%$ & $7.80 \%$ & $7.80 \%$ & $7.81 \%$ & $7.80 \%$ \\
\hline
\end{tabular}

Tabela 3.6: Erro de classificação para os dados de MNIST.

\begin{tabular}{c|c|c|c|c|c|c|c|c}
\hline & $\boldsymbol{1}$ & $\mathbf{5}$ & $\boldsymbol{N}$ & $\boldsymbol{\eta}$ & $\boldsymbol{P}$ & $\mathbf{3}$ & $\boldsymbol{2}$ & $\mathbf{9}$ \\
\hline Classificação real & 1 & 5 & 1 & 7 & 8 & 3 & 2 & 9 \\
Classificação estimada & 2 & 4 & 8 & 0 & 9 & 9 & 8 & 2 (SGD : 0) \\
\hline
\end{tabular}

Tabela 3.7: Exemplo de dígitos erroneamente classificados por todos os algoritmos no problema MNIST 


\section{Capítulo 4}

\section{Conclusões}

Aprendizado de máquina é uma área que atualmente se encontra em grande desenvolvimento inteletual. Basicamente refere-se a um problema de minimização onde existe alguma incerteza nos dados. Nesta dissertação foram estudadas as fundamentações teóricas de alguns algoritmos de otimização estocástica que permitem a resolução do problema, entre eles, o algoritmo clásico Stochastic Gradient Descent, duas metodologias que variam o tamanho amostral a cada iteração e dois algoritmos de redução de variância, todos eles motivados pelo fato de melhorar a estimação do gradiente estocástico.

Os dois exemplos analisados fazem parte do problema de classificação. É importante ressaltar que o objetivo principal deste trabalho não inclui a seleção da melhor função de aproximação, é por isto que a forma funcional escolhida para cada problema é fixa e não foi testada nem comparada com outras possíveis opções. Para cada um dos algoritmos, foram testados diferentes parâmetros que precisam ser definidos a-priori. Este passo é realmente importante, pois a seleção errada de algum deles pode ocasionar que a solução encontrada não seja a melhor para o algoritmo a testar. Pode-se ver o caso do Algoritmo 2.1.2 - SGD que no exemplo de MNIST definindo $\alpha=1$ o erro na amostra de validação pode chegar a ser 10 pontos percentuais maior que o $\alpha$ selecionado.

O método Stochastic Gradient Descent, devido as características que possui o passo de atualização do gradiente, a medida que aumentam as iterações, a modificação do minimizador é pequena,o que garante que depois de certo número de iterações, o minimizador estimado se estabilize. Uma das desvantagens observadas foi a falta de definição de uma condição de parada o que gera a determinação do número de iterações a realizar que pode ocasionar em sobretreinamento dependendo do caso.

Em relação ao Algoritmo 2.2.2 - LSSS, um dos problemas que apresentou foi a grande variação do tamanho amostral nas primeiras iterações, chegando inclusive no número máximo de dados definido $N_{\max }$ e posteriormente diminuindo o seu valor para iniciar um aumento menos acelerado, a pesar de comparar a falta de precisão e quantidade de decrescimento para evitar aumentos desnecessários na minimização. Isto poderia ser evitado se flexibilizamos a condição no Passo 33.2 nas primeiras iterações ou estipulamos uma iteração mínima na qual o aumento do tamanho amostral pode iniciar.

Ao igual que o Algoritmo 2.2.2 - LSSS, a desvantagem do Algoritmo 2.2.1 - DSSS foi atingir o total de dados da amostra de treinamento em poucas iterações, aumentando o custo da metodologia definida como a quantidade de avaliações do gradiente em um único dado $\xi_{i}$ a valores muito altos. Assim, em poucas iterações, o comportamento deste algoritmo é igual a Descida do Gradiente.

Os algoritmos de redução da variância (Algoritmo 2.3.1 - SVRG e Algoritmo 2.3.2 - SAGA) a pesar de continuar apresentando a não diminuição constante na minimização da função objetivo produto da seleção de um único dado amostral para a estimação do gradiente, constataram a convergência no sentido de média quadrada a uma solução próxima do mínimo, chegando inclusive a soluções praticamente igual de precisas que os algoritmos de tamanho amostral variável. Logo, pelo custo e acurácia, estes algoritmos resultaram os mais vantajosos entre os testados.

Na prática, Stochastic Gradient Descent com a generalização de usar a aproximação do gradiente com uma amostra de tamanho $m$ aleatória para cada iteração é um dos algoritmos mais utilizados, 
ao igual que uma variação proposta pelo Nesterov [Nes04] conhecida como Acelerated Gradient Descent. Em trabalhos futuros seria interessante estudar a possibilidade de inclusão de redução de variância no algoritmo do Nesterov, assim como continuar analisando a definição de uma condição de parada que seja compatível com a convergência em probabilidade que os algoritmos possuem. 


\section{Referências Bibliográficas}

[AG07] Søren Asmussen e Peter W. Glynn. Stochastic Simulation: Algorithms and Analysis. Springer, 2007. 2, 11, 20

[AMMIL12] Yaser S. Abu-Mostafa, Malik Magdon-Ismail e Hsuan-Tien Lin. Learning from Data. AML Book, 2012. 37, 40

[BCNW12] Richard H. Byrd, Gillian M. Chin, Jorge Nocedal e Yuchen Wu. Sample Size Selection in Optimization Methods for Machine Learning. Mathematical Programming, 134(1):127-155, 2012. 2, 12, 13, 14

[Che03] Han-Fu Chen. Stochastic Approximation and its applications. Kluwer Academic Publishers, 2003. 6

[DBLJ14] Aaron Defazio, Francis Bach e Simon Lacoste-Julien. SAGA: A fast incremental gradient method with support for Non-Strongly Convex Composite Objectives. Advances in Neural Information Processing Systems, páginas 1646-1654, 2014. 2, 25, 26, 28, 35

[DeG89] Morris H. DeGroot. Probability and Statistic. Addison-Wesley Publishing Company, 2nd edição, 1989. 3

[eSW10] Jorge Nocedal e Stephen Wright. Numerical Optimization. Springer, 2nd edição, 2010. 43

[Hay05] Simon Haykin. Neural Networks. A comprehensive foundation. Pearson Prentice Hall, 2nd edition edição, 2005. 40

[HTF08] Trevor Hastie, Robert Tibshirani e Jerome Friedman. The Elements of Statistical Learning: Data Mining, Inference, and Prediction. Springer, 2nd edition edição, 2008. 39

[JZ13] Rie Johnson e Tong Zhang. Accelarating Stochastic Gradient Descent using Predictive variance reduction. Advances in Neural Information Processing Systems, 26:315-323, 2013. $2,21,23$

[KK13] Nataŝa Krejić e Nataŝa Krklec. Line search methods with variable sample size for uncontrained optimization. Journal of Computational and Applied Mathematics, 245:213231, 2013. 2, 6, 11, 12, 15, 17, 19

[KKK10] Ralf Korn, Elke Korn e Gerald Kroisandt. Monte Carlo Methods and Models in Finance and Insurance. CRC Press and Taylor \& Francis Group, 2010. 2, 11, 20

[KLS13] Nataŝa Krejić, Zorana Luẑanin e Irena Stojkvska. A gradient method for unconstrained optimization in noisy environment. Applied Numerical Mathemtics, 70:1-21, 2013. 2

[KM16] Nataŝa Krejić e Jose Mario Martinez. Inexact Restoration approach for minimization with inexact evaluation of the objective function. Mathematics of Computations, 85:1775-1791, 2016. 2 
[LBBH98] Yann LeCun, Léon Bottou, Yoshua Bengio e Patrick Haffner. Gradient-based learning applied to document recognition. Proceedings of the IEEE, 86(11):2278-2324, 1998. 43

[MRT12] Mehryar Mohri, Afshin Rostamizadeh e Ameet Talwalkar. Foundations of Machine Learning. The MIT Press, 2012. 1

[Nes04] Yurii Nesterov. Introductory Lectures on Convex Optimization: A Basic Course. Springer, 2004. 50

[Pol87] Boris T. Polyak. Introduction to optimization. Optimization Software, Inc. Publications Division, 1987. 7

[Rip87] Brian D. Ripley. Stochastic Simulation. John Wiley \& Sons Inc, 1987. 2, 20

[RM51] Herbert Robbins e Sutton Monro. A stochastic approximation method. The Annals of Mathematical Statistic, 22(3):400-407, 1951. 1, 5

[Ros97] Sheldon Ross. A first course in probability. Prentice Hall, 5th edition edição, 1997. 3, 12

[Ros10] Sheldon Ross. Introduction to probability models. Academic Press, 10th edition edição, 2010. 3

[RS71] Herbert Robbins e David Siegmund. A convergence theorem for non negative almost supermartingales and some applications. Optimizing methods in Statitics, 1971. 6

[Spa03] James C. Spall. Introduction to Stochastic Search and Optimization. Wiley-Iterscience series in discrete mathematics, 2003. 1, 10

[YNS12] Farzad Yousefian, Angelia Nedić e Uday V. Shanbhag. On Stochastic Gradient and Subgradient Methods with Adaptive Steplength Sequences. Automatica, 48(1):56-67, 2012. 10 